Check for updates

Cite this: DOI: $10.1039 / \mathrm{c} 9 \mathrm{cs} 00432 \mathrm{~g}$

\section{Emerging new phase boundary in potassium sodium-niobate based ceramics}

\author{
Xiang Lv, (D) ab Jianguo Zhu, ${ }^{a}$ Dingquan Xiao, ${ }^{a}$ Xi-xiang Zhang ${ }^{~}{ }^{b}$ and Jiagang Wu (D) *a
}

Developing eco-friendly high-performance piezoceramics without lead has become one of the most advanced frontiers in interdisciplinary research. Although potassium sodium-niobate $\left\{(\mathrm{K}, \mathrm{Na}) \mathrm{NbO}_{3}, \mathrm{KNN}\right\}$ based ceramics are believed to be one of the most promising lead-free candidates, the relatively inferior piezoelectric properties and strong temperature dependency have hindered their development for more than 50 years since being discovered in the 1950s. It was not until 2014 that our group initially proposed a new phase boundary (NPB) that simultaneously improved the piezoelectric properties and temperature stability of non-textured KNN-based ceramics to the level of partly lead-based ceramics. The NPB has been then proved by some researchers and believed to pave the way for "lead-free at last" proposed by E. Cross (Nature, 2004, 432, 24). However, the understanding of the NPB is still in its infancy, leaving many controversies, including the phase structure and physical mechanisms at the NPB as well as the essential difference when compared with other phase boundaries. In this context, we systematically summarized the origin and development of the NPB, focusing on the construction, structure and intrinsic trait of the NPB, the effects of the NPB on the performance, and the validity and related incipient devices of the NPB. Particularly, we concluded the phase structure and domain structure locating at the NPB, analyzed the physical mechanisms in depth, proposed the possible methods to further improve the performance at the NPB, and demonstrated the validity and scope of the NPB as well as the device application. Finally, we gave out our perspective on the challenges and future research of KNN-based ceramics with NPB. Therefore, we believe that this review could promote the

understanding of the NPB and guide the future work of KNN-based ceramics.
Received 19th September 2019

DOI: $10.1039 / \mathrm{c} 9 \mathrm{cs} 00432 \mathrm{~g}$

rsc.li/chem-soc-rev

${ }^{a}$ Department of Materials Science, Sichuan University, Chengdu, 610065, P. R. China. E-mail: wujiagang0208@163.com, msewujg@scu.edu.cn

${ }^{b}$ Division of Physical Science and Engineering, King Abdullah University of Science and Technology (KAUST), Thuwal, 239955, Kingdom of Saudi Arabia

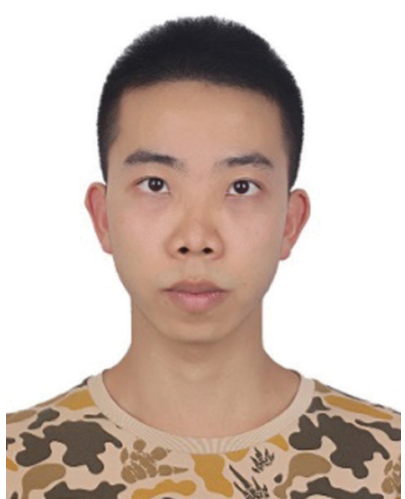

Xiang Lv
Xiang $L v$ received his $B S$ and $P h D$ degrees from Sichuan University in 2015 and 2019. He finished his PhD thesis under the guidance of Prof. Jiagang Wu and Prof. Jianguo Zhu. His research interest concentrates on the relationship of "composition design-phase/micro structure-performance-physical mechanism" in lead-free ferro/piezoelectric ceramics. He has published 19 papers as the first author or corresponding author. Currently, he is a postdoctoral fellow at King Abdullah University of Science and Technology (KAUST).

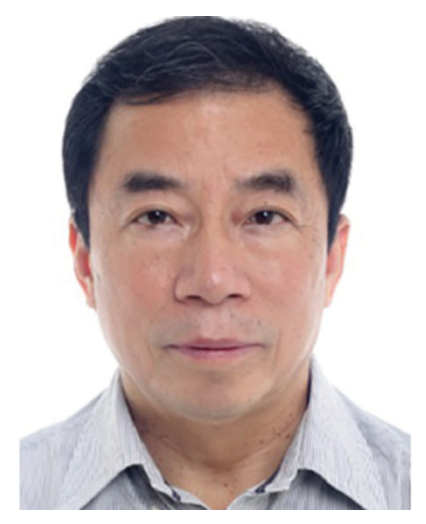

Jianguo Zhu
Prof. Jianguo Zhu obtained his MS and $P h D$ degrees in Physics from Sichuan University in 1987 and 1998, respectively. During 19871988, he worked as an assistant professor at the Department of Physics, Sichuan University. From 1988 to now, he has worked at the Department of Materials Science, Sichuan University as an assistant professor, associated professor, and professor. His research interests mainly focus on the controllable-synthesis, characterization, and properties of ferro- 1 piezo-/dielectric functional materials. He is the author of over 300 peerreviewed articles, 9 books, and over 20 Chinese patents and has given about 50 invited lectures in various meetings and conferences. 


\section{Background}

Owing to the exceptional ability of interconverting mechanical energy and electrical energy, piezoelectric materials, represented by lead zirconate titanate $\left\{(\mathrm{Pb}, \mathrm{Zr}) \mathrm{TiO}_{3}, \mathrm{PZT}\right\}$ and relaxor- $\mathrm{PbTiO}_{3}$ $\left\{\mathrm{Pb}\left(\mathrm{Mg}_{1 / 3} \mathrm{Nb}_{2 / 3}\right) \mathrm{O}_{3}, \mathrm{PMN}-\mathrm{PT}\right\}$ family, are widely employed in different areas, such as traditional electronic devices (e.g., actuators, ultrasonic transducers, buzzers and sensors) and emerging fields (e.g., energy harvesting and storage, nanodevices, biomedicine, and micro-electromechanical systems, MEMS). ${ }^{1-18}$ However, the increasing demand for environmental protection and sustainable development strictly restricts the use or content of toxic element lead $(\mathrm{Pb})$ in electronic devices, resulting in the rapid development of lead-free piezoceramics that are represented by barium titanate $\left\{\mathrm{BaTiO}_{3}, \mathrm{BT}\right\}$, potassium sodium niobate $\left\{(\mathrm{K}, \mathrm{Na}) \mathrm{NbO}_{3}, \mathrm{KNN}\right\}$, bismuth ferrite $\left\{\mathrm{BiFeO}_{3}, \mathrm{BFO}\right\}$ and sodium bismuth titanate $\left\{\left(\mathrm{Bi}_{1 / 2} \mathrm{Na}_{1 / 2}\right) \mathrm{TiO}_{3}, \mathrm{BNT}\right\} .{ }^{5,9,11,19,20}$ Recently, the

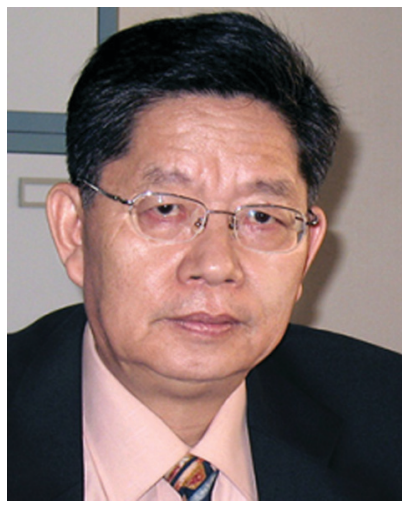

Dingquan Xiao
Prof. Dingquan Xiao graduated from Sichuan University as an undergraduate in 1968 and as a postgraduate in 1980. Then, he studied in Queen Mary College, University of London from 1980 to 1982, and worked as a visiting professor at Penn State University in 1990 and at the University of Houston in 1998. He started to work at Sichuan University from 1983. He was a member of the National Advanced Materials Committee of China and a member of the Chinese Materials Research Society and also the Chinese Physics Society. He has published more than 200 papers in English journals since 2000.

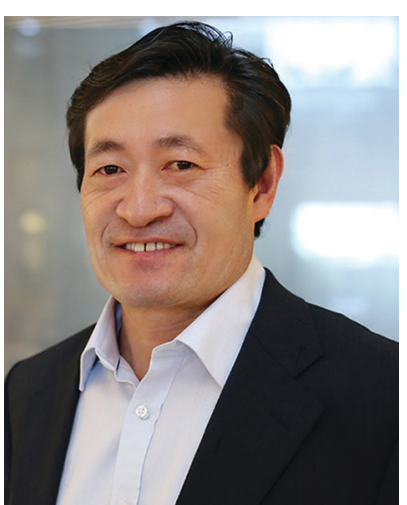

Xi-xiang Zhang
Prof. Xi-xiang Zhang obtained his $\mathrm{PhD}$ degree from the University of Barcelona, Spain, in 1992. After working as a research scientist at Department de Fisica Fonamental, University de Barcelona for five years (1992-1997), he joined Hong Kong University of Science and Technology as an assistant professor, and then became a full-time professor in July 2008. In September 2008, he joined KAUST as the manager/ director of the core lab and became 2014. His research interests include magnetism, spintronics, nanomaterials, multiferroic materials, two-dimensional (2D) materials and graphene. He is a fellow of the American Physical Society. a full-time professor in January renewal of Restriction of the use of certain Hazardous Substances (e.g., RoHS 2) declared that “...information available does not allow excluding that lead-free solutions for ceramics are or will become available within less than 5 years". RoHS 2 also stated that the replacement of PZT “...may be scientifically and technologically practical to a certain degree...”, although replacement "... is scientifically and technically still impractical in the majority of applications". ${ }^{13}$ Therefore, it is urgent to develop high-performance lead-free piezoceramics to replace the lead-based ones. Endowed with moderate piezoelectricity (piezoelectric coefficient, $d_{33}=80-120 \mathrm{pC} \mathrm{N}^{-1}$ ) and high Curie temperature $\left(T_{\mathrm{c}}=410{ }^{\circ} \mathrm{C}\right)$, pure $\mathrm{KNN}$ ceramics have attracted the researchers' eyes. ${ }^{21}$ The last twenty years have witnessed the rapid development of $\mathrm{KNN}$-based ceramics, particularly with respect to their piezoelectricity enhancement. ${ }^{14}$ The significant progress is mainly attributed to the development of the phase boundary. ${ }^{10}$ The early work on the phase boundary mainly focused on the study of the orthorhombic-tetragonal (O-T) phase boundary, which was initiated by Saito et al. ${ }^{22}$ Although the $\mathrm{O}-\mathrm{T}$ phase boundary could improve the piezoelectric properties to some degree, the obvious deficiencies still existed, that is, the relatively low piezoelectric properties compared to those of commercial lead-based ceramics and the strong temperature dependence of piezoelectric properties. ${ }^{6,14}$ Recently, the emerging new phase boundary (NPB), which was constructed by simultaneously shifting rhombohedral-orthorhombic and orthorhombictetragonal phase transition temperature points (e.g., $T_{\mathrm{R}-\mathrm{O}}$ and $\left.T_{\mathrm{O}-\mathrm{T}}\right)$ to or near room temperature, was reported to substantially improve $d_{33}$ values of non-textured KNN-based ceramics to $490-650 \mathrm{pC} \mathrm{N}^{-1}$, which are even comparable to those of commercial soft lead-based ceramics (e.g., $d_{33}$ values of $410 \mathrm{pC} \mathrm{N}^{-1}$ and $590 \mathrm{pC} \mathrm{N}^{-1}$ for PZT-4 and PZT-5H ceramics, respectively). ${ }^{10,23,24}$ In addition, enhanced strain properties and improved temperature stability were also observed in KNN-based ceramics with NPB. ${ }^{25-27}$

The previous reviews (before 2018) have systematically summarized the relationship of "chemical modification-phase

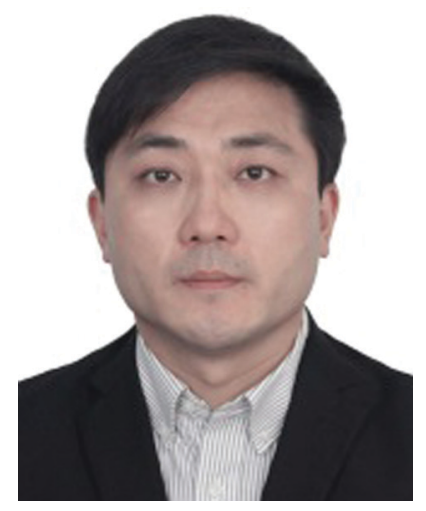

Jiagang Wu
Prof. Jiagang $W u$ has been a fulltime professor at Sichuan University since 2015. He received his BS and $\mathrm{PhD}$ degrees in Materials Physics and Chemistry from Sichuan University in 2003 and 2008, respectively. Then, he joined Prof. John Wang's group (Department of Materials Science and Engineering of National University of Singapore) as a Singapore Millennium Postdoctoral Fellow (SMF-PDF) from 2008 to 2010. His research interest mainly concentrates on the relationship of "structure-composition design-property modification" in ferro/piezoelectric/multiferroic materials, especially in lead-free piezoceramics. He has published more than 160 papers in the field of ferro/piezoelectric materials as the first author or corresponding author. 
boundaries-piezoelectric properties", which particularly focused on the $\mathrm{O}-\mathrm{T}$ phase boundary, but briefly mentioned or summarized the NPB in the initial stage. ${ }^{2,5,9-11}$ But however, the KNN-based ceramics with NPB exhibited much better performance (including piezoelectric properties and temperature stability) compared to those with O-T phase boundary. ${ }^{5,6,10,11,15,16,23,24,26,28,29}$ Besides the piezoelectric properties, the strain properties and the temperature stability are also important factors for practical applications, which are exactly the disadvantages of KNN-based ceramics. ${ }^{9,10}$ Although the recently reported reviews focused on the piezoelectric properties and temperature stability of KNN-based ceramics with NPB, they lacked in-depth analysis of physical mechanisms, such as the discussions of phase structure and domain configuration, the intrinsic trait of the NPB, the difference between NPB and other phase boundaries, and the fundamental understanding of temperature stability. ${ }^{14-16}$ More importantly, these reviews did not provide the possible methods for further improving the performance in the future. ${ }^{14-16}$ The lack of these fundamental but important analyses and considerations seriously hinders the understanding of the NPB and the development of KNN-based ceramics for industrial applications. Particularly, the recent publications suggested the significant advance of KNN-based ceramics with NPB in performance, incipient devices, and physical mechanisms, ${ }^{24,29-36}$ indicating that the NPB could endow KNN-based ceramics with potential applications. Unfortunately, not enough considerations were given to the NPB of KNN-based ceramics in the previous reviews, ${ }^{5,6,9,10,14-16}$ which greatly hindered the further development of the NPB in KNN-based ceramics.

Stimulated by the demand for developing high-performance lead-free piezoceramics and considering the excellent performance of KNN-based ceramics with NPB, it is necessary to systematically review the newly developed NPB, which is absent but will effectively guide the current and future research of KNN-based ceramics. In this review, we took the NPB as the core objective and systematically summarized the origin, development, and future of the NPB in KNN-based ceramics, particularly focusing on the development of the NPB, the intrinsic trait of the NPB, the effects of the NPB on the structure (e.g., phase, domain, and heterogeneity) and performance (e.g., piezoelectric properties, strain properties and temperature stability) of KNN-based ceramics, and the related physical mechanisms by simultaneously considering both intrinsic and extrinsic contributions. Subsequently, the validity of the NPB and the related incipient devices were addressed. Finally, we provided some perspectives on the challenges and future research of KNN-based ceramics with NPB. The detailed structure chart of this review is provided in Fig. 1. We believe that this comprehensive review will provide new insights into developing the state-of-the-art KNN-based ceramics with comparable performance to those of lead-based ones.

\section{Characteristics of the NPB}

\subsection{History of the phase boundary}

Among these lead-free piezoceramics candidates, KNN ceramics have been widely investigated owing to the moderate piezoelectricity

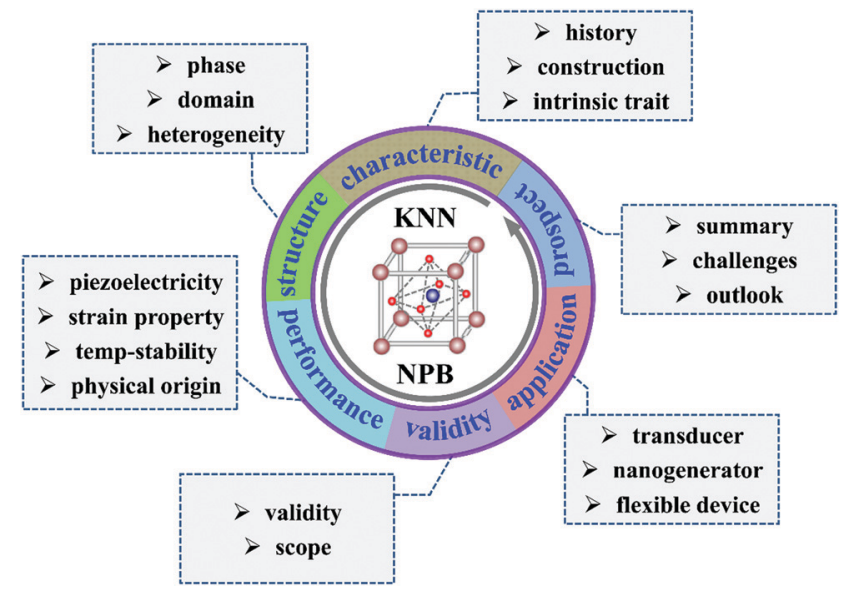

Fig. 1 Structure chart of this review.

(e.g., $\left.d_{33}=80-160 \mathrm{pC} \mathrm{N}^{-1}\right)$ and high $T_{\mathrm{c}}\left(\sim 410{ }^{\circ} \mathrm{C}\right) .{ }^{21}$ Before 2004, the development of lead-free piezoelectric materials was extremely slow due to the absence of promising piezoelectric properties (see Fig. 2(a)). In 2004, Saito et al. firstly reported highly textured LF4T ceramics with comparable performance (e.g., $d_{33}$ and temperature stability) to that of commercial PZT-4 ceramics, ${ }^{22}$ which ignited the passion for research on lead-free piezoelectric materials. Then, the research on $\mathrm{KNN}$-based ceramics rapidly developed with each passing year, reaching the relative peak stage in 2016-2018 (see Fig. 2(a)). The rapid development of KNN-based ceramics was mainly attributed to phase boundary engineering, which can significantly improve the piezoelectric properties and endow KNN-based ceramics with promising potential for practical applications. ${ }^{10,14}$ Currently, KNN-based ceramics share a percentage as high as $43.85 \%$ in lead-free piezoelectric ceramics, while the investigations on phase boundaries occupy a high percentage of $53.27 \%$ in KNN-based ceramics (see Fig. 2(b)). Actually, the investigations on phase boundaries are also dominating in other lead-free piezoceramics, including BT-, BFO- and BNT-based ceramics. ${ }^{12,14,15,20,37-39}$ Therefore, the phase boundary has become the most widely studied theme in lead-free piezoceramics, which is claimed to be the most useful tool to modify the performance. ${ }^{10,12,20}$

Fig. 2(c) shows the historical evolution of phase boundaries and related performance in KNN-based ceramics. The beginning of KNN-based ceramics' phase boundaries can be traced back to 2004. Through the O-T phase boundary and the reaction template grain growth (RTGG) method, Saito et al. significantly improved the performance of KNN-based ceramics up to the level of commercial PZT-4 ceramics. $^{22}$ Then, the researchers started to construct the same or similar O-T phase boundary in KNN-based ceramics, with the purpose of pursuing comparable performance to those of commercial lead-based counterparts. ${ }^{10}$ Unfortunately, the results showed that $d_{33}$ values of non-textured KNN-based ceramics with $\mathrm{O}-\mathrm{T}$ phase boundary were limited below $416 \mathrm{pC} \mathrm{N}^{-1}$, inferior to those of other commercial leadbased ceramics. ${ }^{10}$ Although moderate piezoelectric properties were still obtained in $\mathrm{KNN}$-based ceramics with $\mathrm{O}-\mathrm{T}$ phase boundary, the exploration for constructing $\mathrm{O}-\mathrm{T}$ phase boundary 


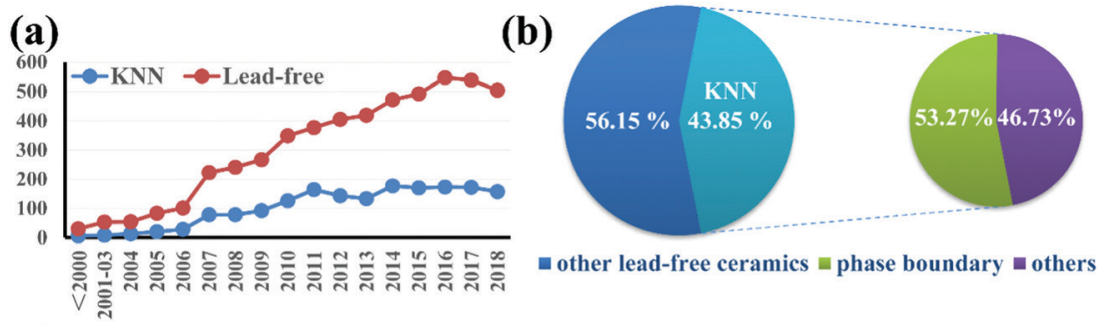

(c)

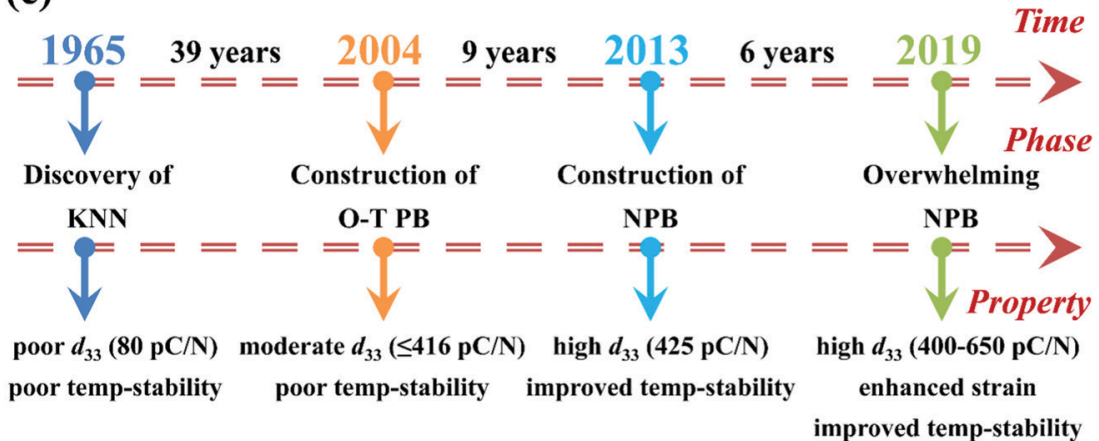

Fig. 2 Development of KNN-based ceramics and their phase boundaries. (a) Publications of lead-free piezoelectric (red column) and KNN-based materials (blue column). The data were collected from the ISI Web of Science using the keywords of "lead-free" and "piezoelectric" for lead-free piezoelectric, and "KNN", "potassium sodium niobate" as well as "piezoelectric" for KNN-based materials. (b) Current investigations of lead-free ceramics and KNN-based ceramics. (c) Historical evolutions of the phase boundary and related performance in KNN-based ceramics.

accumulated the abundant experience for selecting potential additives that can effectively tailor $T_{\mathrm{O}-\mathrm{T}}$ of KNN ceramics. ${ }^{10,14}$

In 2009, some researchers unconsciously shifted $T_{\mathrm{R}-\mathrm{O}}$ and $T_{\mathrm{O}-\mathrm{T}}$ towards room temperature, resulting in the increased $d_{33}$ of $300-400 \mathrm{pC} \mathrm{N} \mathrm{N}^{-1} \cdot{ }^{40}$ Unfortunately, the variations of $T_{\mathrm{R}-\mathrm{O}}$ with additives were ignored at that time due to the low temperature (e.g., $\left.<-100{ }^{\circ} \mathrm{C}\right) .{ }^{40}$ It was not until 2013 that $T_{\mathrm{R}-\mathrm{O}}$ of KNN-based ceramics was paid much attention. At that time, our group initially observed the variations of $T_{\mathrm{R}-\mathrm{O}}$ and $T_{\mathrm{O}-\mathrm{T}}$ as a function of additives' content. ${ }^{41-43}$ It was found that appropriate additives can simultaneously shift $T_{\mathrm{R}-\mathrm{O}}$ and $T_{\mathrm{O}-\mathrm{T}}$ to or near room temperature, forming a new phase boundary (NPB) that is totally different from the O-T phase boundary. Subsequently, enhanced piezoelectric properties (e.g., $d_{33}=400-650 \mathrm{pC} \mathrm{N}^{-1}$ ), improved strain properties as well as favorable temperature stability were continuously reported in KNN-based ceramics with NPB. ${ }^{23-26,44-46}$ Therefore, the NPB occupies the overwhelming majority of investigations on phase boundaries in KNN-based ceramics at present. ${ }^{10,14}$

\subsection{Construction of the NPB}

Fig. 3(a) shows the temperature-dependent dielectric permittivity $\left(\varepsilon_{\mathrm{r}}-T\right)$ curve of pristine $\mathrm{K}_{0.5} \mathrm{Na}_{0.5} \mathrm{NbO}_{3}$ ceramics. Three phase transitions are clearly observed from low to high temperature, that is, rhombohedral-orthorhombic phase transition (R-O), orthorhombic-tetragonal phase transition $(\mathrm{O}-\mathrm{T})$, and tetragonal-cubic phase transition $(\mathrm{T}-\mathrm{C})$. Their phase transition temperature points are named as $T_{\mathrm{R}-\mathrm{O}}, T_{\mathrm{O}-\mathrm{T}}$, and $T_{\mathrm{c}}$, respectively. ${ }^{6}$ Previously, the researchers mainly shifted $T_{\mathrm{O}-\mathrm{T}}$ to or near room temperature for constructing the $\mathrm{O}-\mathrm{T}$ phase boundary, which improved $d_{33}$ values of non-textured KNN-based ceramics to
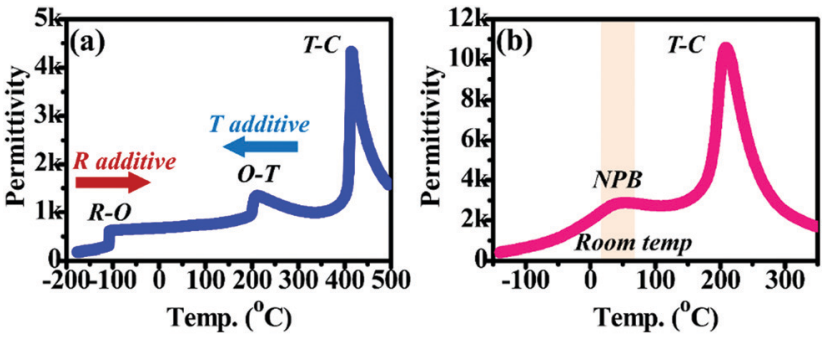

Fig. 3 Schematic diagram of constructing the new phase boundary. Temperature-dependent permittivity $\left(\varepsilon_{\mathrm{r}}-T\right)$ curve of (a) pure $\mathrm{K}_{0.5} \mathrm{Na}_{0.5} \mathrm{NbO}_{3}$ ceramics and (b) KNN-based ceramics with NPB. With the help of additives, both $T_{\mathrm{R}-\mathrm{O}}$ and $T_{\mathrm{O}-\mathrm{T}}$ shift to room temperature, resulting in the NPB.

$400 \mathrm{pC} \mathrm{N}{ }^{-1} .^{40}$ However, the NPB was constructed by simultaneously shifting $T_{\mathrm{R}-\mathrm{O}}$ and $T_{\mathrm{O}-\mathrm{T}}$ to or near room temperature (see Fig. 3(b)). Thus, choosing the appropriate additives and tailoring their content are the most important factors for constructing the NPB. Our previous review has systematically concluded the possible additives that can effectively shift $T_{\mathrm{R}-\mathrm{O}}$ and $T_{\mathrm{O}-\mathrm{T}}$ of KNN ceramics, that is, $\mathrm{A} / \mathrm{B}$-site ion substitution and $\mathrm{ABO}_{3}$ type multi-component substitution. ${ }^{10,14}$ Table 1 lists the additives reported in the literature. It should be pointed out that Table 1 only gives out the original formula of additives, which could be modified by using other equivalent or non-equivalent ions. For example, one can modify " $\mathrm{M}$ " of $\left(\mathrm{Bi}_{0.5} \mathrm{M}_{0.5}\right)^{2+}$ by combining two or more types of " $\mathrm{M}$ " ions (e.g., $\left.\mathrm{Bi}_{0.5}\left(\mathrm{~K}_{0.18} \mathrm{Na}_{0.82}\right)_{0.5}{ }^{2+}\right)$ as well as partially substitute the $\mathrm{B}$ ion of the $\mathrm{ABO}_{3}$ type component by using an equivalent ion (e.g., $\left.\left(\mathrm{Bi}_{0.5} \mathrm{Na}_{0.5}\right)\left(\mathrm{Hf}_{0.9} \mathrm{Sn}_{0.1}\right) \mathrm{O}_{3}\right) .^{31,44}$ Moreover, we can also simply calculate the effect of these additives on the shifting of $T_{\mathrm{R}-\mathrm{O}}$ and $T_{\mathrm{O}-\mathrm{T}}$ of KNN ceramics, 
Table 1 Reported additives for tailoring $T_{\mathrm{R}-\mathrm{O}}$ and $T_{\mathrm{O}-\mathrm{T}}$ of $\mathrm{KNN}$ ceramics in the literature

\begin{tabular}{|c|c|c|c|c|}
\hline \multicolumn{2}{|l|}{ Substitution } & \multicolumn{3}{|c|}{$\mathrm{ABO}_{3}$ component } \\
\hline$\overline{\mathrm{Li}^{+}, \mathrm{Ag}^{+}, \mathrm{K}^{+} / \mathrm{Na}^{+}}$ & $\mathrm{Sb}^{5+}, \mathrm{Ta}^{5+}$ & $\mathrm{A}^{3+} \mathrm{B}^{3+} \mathrm{O}_{3}$ & $\begin{array}{l}\mathrm{Zr}^{4+}, \mathrm{Hf}^{4+}, \mathrm{Ti}^{4+} \\
\mathrm{Sn}^{4+} \\
\mathrm{Fe}^{3+}, \mathrm{Al}^{3+}, \mathrm{Sc}^{3+}, \mathrm{Co}^{3+}, \mathrm{Ga}^{3+}\end{array}$ & $\begin{array}{l}\mathrm{Ba}^{2+}, \mathrm{Sr}^{2+}, \mathrm{Ca}^{2+},\left(\mathrm{Bi}_{0.5} \mathrm{M}_{0.5}\right)^{2+}, \mathrm{M}=\mathrm{K}^{+}, \mathrm{Na}^{+}, \mathrm{Ag}^{+}, \mathrm{Li}^{+}, \\
\text {and their combinations } \\
\mathrm{Ba}^{2+}, \mathrm{Ca}^{2+} \\
\mathrm{Bi}^{3+}\end{array}$ \\
\hline
\end{tabular}

Table 2 Shifting rate of reported additives on $T_{\mathrm{R}-\mathrm{O}}, T_{\mathrm{O}-\mathrm{T}}$, and $T_{\mathrm{C}}$ of KNN ceramics ${ }^{a}$

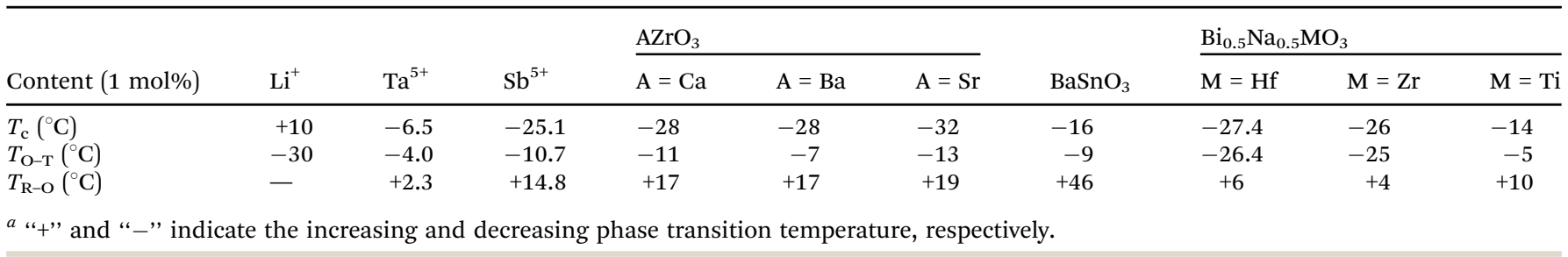

as listed in Table $2 .^{28,47-57}$ One can see that most additives could reduce $T_{\mathrm{c}}$ of $\mathrm{KNN}$ ceramics expect for $\mathrm{Li}^{+}$, which is the reason why $\mathrm{Li}^{+}$substitution was largely studied for constructing the O-T phase boundary. ${ }^{6,10,58}$ However, one should bear in mind that the exact tailoring ability of a certain additive may be affected by other co-doping additives. That is the reason why a similar NPB can be constructed in various formulas with different contents of additives. ${ }^{59,60}$ Therefore, the process of choosing appropriate additives and tailoring their content should be as cautious as possible for constructing the NPB.

\subsection{Intrinsic trait of the NPB}

As mentioned above, the tunability of $T_{\mathrm{R}-\mathrm{O}}$ and $T_{\mathrm{O}-\mathrm{T}}$ makes it possible for KNN-based ceramics to possess the phase boundaries (e.g., O-T phase boundary and $\mathrm{NPB}$ ) at room temperature. Therefore, the phase structure of KNN-based ceramics not only depends on the composition but also ambient temperature (see Fig. 4(a)). Such a trait means that the enhanced piezoelectricity will be influenced when the ambient temperature goes up (down) to a level higher (lower) than that of the phase boundary, resulting in the obvious temperature dependence of piezoelectricity that is not good for practical applications. Therefore, the researchers treated this behavior as the polymorphic phase boundary (PPB) (see Fig. 4(a)), which is different from the composition-dependent morphotropic phase boundary (MPB)
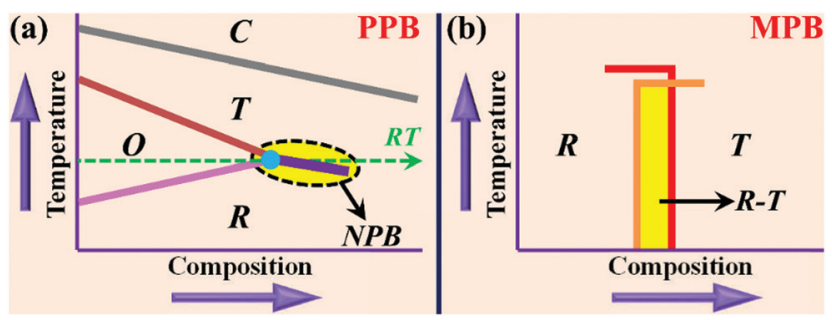

Fig. 4 Intrinsic traits of the polymorphic phase boundary (PPB) and morphotropic phase boundary (MPB). (a) General phase diagram for constructing the NPB. (b) Phase diagram of a typical MPB. reported in PZT ceramics (see Fig. 4(b)). ${ }^{1,61}$ Obviously, the PPB is bound by both composition and temperature, while the MPB is only influenced by composition when $T<T_{\mathrm{c}}$. Therefore, the phase boundary of KNN-based ceramics can only be constructed at a given composition and temperature. But however, the practical applications of piezoceramics require both high piezoelectricity and decent temperature stability, which seems to be a contradiction relationship in KNN-based ceramics. ${ }^{8,9}$ Therefore, the state-of-the-art research on KNN-based ceramics mainly concentrates on seeking an effective way to balance their relationship, such as constructing the diffused (or broadened) phase boundary, adding metallic oxide $\left(\mathrm{MnO}_{2}\right)$, and composite ceramic, which will be discussed later. ${ }^{27,29,31,62,63}$

Since both O-T phase boundary and NPB belong to the PPB, one may be eager to know the difference between them, which has perplexed the researchers for more than five years. Herein, we emphatically addressed the differences between them. Generally, it is difficult to clearly read $T_{\mathrm{R}-\mathrm{O}}$ in the temperature-dependent relative dielectric permittivity $\left(\varepsilon_{\mathrm{r}}-T\right)$ curves of unpoled KNN-based ceramics. ${ }^{28,64-67}$ Recently, our group and Zhang et al. found that $T_{\mathrm{R}-\mathrm{O}}$ can be clearly read from the temperature-dependent imaginary part of dielectric permittivity $\left(\varepsilon^{\prime \prime}-T\right)$ curves of poled KNN-based ceramics. ${ }^{32,68,69}$ Fig. 5(a) exhibits the phase diagram of poled KNNS-SZ- $x$ BAZ ceramics that were designed for constructing a typical NPB. ${ }^{30,32}$ With increasing $x, T_{\mathrm{R}-\mathrm{O}}$ first slightly increased and then disappeared at $x=0.04$, while $T_{\mathrm{O}-\mathrm{T}}$ reduced monotonously. When further looking at their $\varepsilon^{\prime \prime}-T$ curves, $T_{\mathrm{O}-\mathrm{T}}$ values of the compositions $(x=0.025$ and 0.03$)$ were close to room temperature, while their $T_{\mathrm{R}-\mathrm{O}}$ values were far away from room temperature (see Fig. 5(b) and (c)). Therefore, the phase structure of the two compositions was reasonably identified as an orthorhombic-tetragonal $(\mathrm{O}-\mathrm{T})$ phase coexistence. Their XRD patterns further proved this identification. Two peaks in the $\{200\}_{\mathrm{pc}}$ diffraction of the composition $(x=0.025)$ exhibited a similar intensity, which evidenced the $\mathrm{O}-\mathrm{T}$ phase coexistence (see Fig. 5(e)). ${ }^{67,70}$ On the other hand, the composition $(x=0.03)$ possessed the $\{200\}_{\mathrm{pc}}$ diffraction in which the $(200)_{\mathrm{pc}}$ peak was much higher than the $(002)_{\mathrm{pc}}$ peak because $T_{\mathrm{O}-\mathrm{T}}$ was closer to 

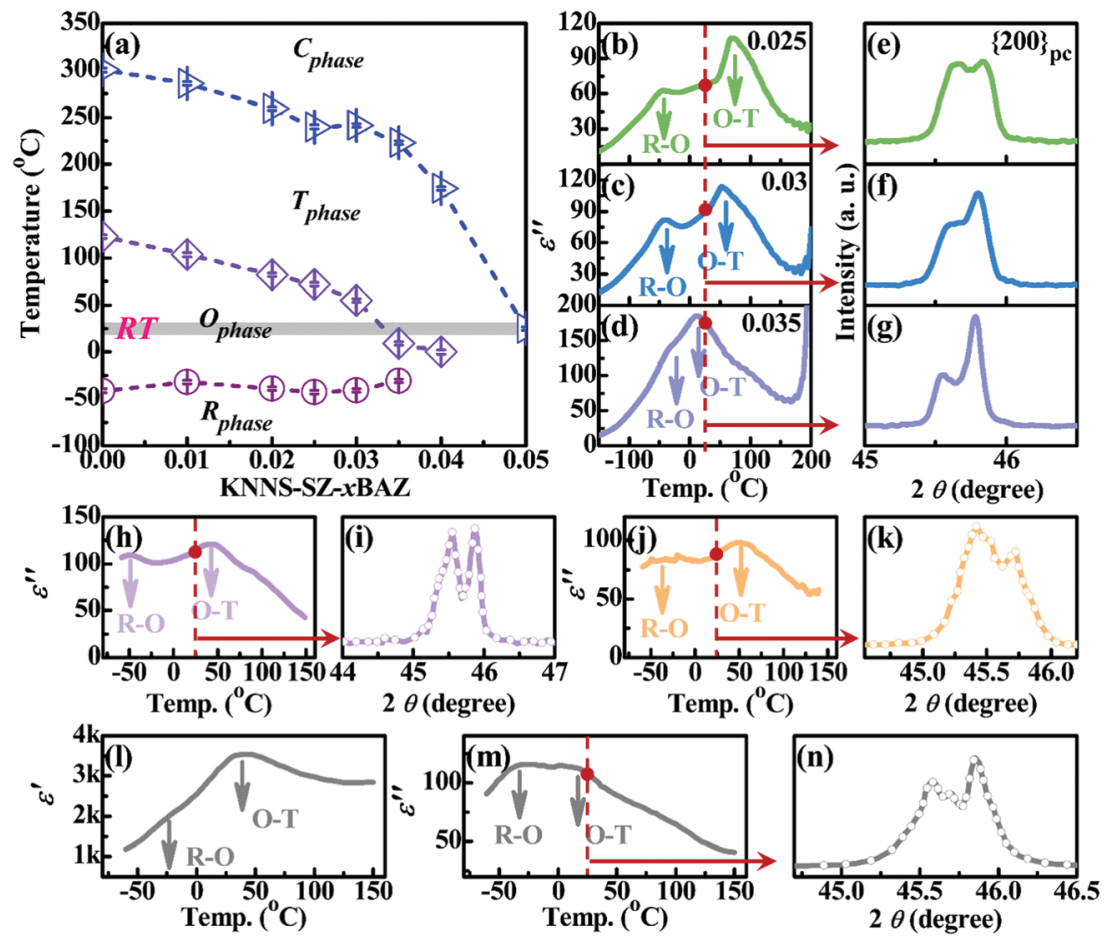

Fig. 5 (a) Phase diagram of poled KNNS-SZ-xBAZ ceramics. Poled $\varepsilon^{\prime \prime}-T$ curve and unpoled room-temperature XRD pattern of KNNS-SZ- $x$ BAZ ceramics with ( $\mathrm{b}$ and e) $x=0.025$, (c and f) $x=0.03$, and ( $\mathrm{d}$ and $\mathrm{g}$ ) $x=0.025$. Poled $\varepsilon^{\prime \prime}-T$ curve and unpoled room-temperature XRD pattern of (h and i) $\mathrm{KNNS}_{0.04}-0.04 \mathrm{BNZ}$ and $\left(\mathrm{j}\right.$ and $\mathrm{k}$ ) KNNS $\mathrm{KN}_{0.045}-0.0375 \mathrm{BNH}$ ceramics. ( $\mathrm{l}$ and $\mathrm{m}$ ) Poled $\varepsilon^{\prime}-T$ and $\varepsilon^{\prime \prime}-T$ curves and (n) unpoled XRD pattern of KNNS $0.04-$ $0.045 \mathrm{BNH}$ ceramics. The dashed line indicates the location of room temperature. (a-g) Reproduced from ref. 32 with permission from the Royal Society of Chemistry, Copyright 2019; ( $h$ and i) reproduced from ref. 68 with permission from the American Institute of Physics, Copyright 2019; (j and k) reproduced from ref. 69 with permission from Elsevier, Copyright 2019; (l-n) reproduced from ref. 72 with permission from Elsevier, Copyright 2019.

room temperature (see Fig. 5(f)), indicating the increasing content of the $\mathrm{T}$ phase within the coexisting $\mathrm{O}-\mathrm{T}$ phases. ${ }^{71}$ But however, $T_{\mathrm{R}-\mathrm{O}}$ of the composition $(x=0.035)$ became indiscernible and diffused towards room temperature, while $T_{\mathrm{O}-\mathrm{T}}$ was slightly lower than room temperature, resulting in the almost converged $T_{\mathrm{R}-\mathrm{O} \& \mathrm{O}-\mathrm{T}}$ (see Fig. $5(\mathrm{~d})$ ). That is to say, the $\mathrm{R}$ phase has diffused into room temperature. Thus, the composition $(x=0.035)$ possessed the $\mathrm{R}-\mathrm{O}-\mathrm{T}$ multi-phase coexistence, in which the $\mathrm{O}$ and $\mathrm{T}$ phases shared the majority and the $\mathrm{R}$ phase existed in the form of a "diffused" phase. ${ }^{32}$ The XRD pattern showed a further increase in the relative intensity of the $(200)_{\mathrm{pc}}$ peak due to $T_{\mathrm{O}-\mathrm{T}}$ being slightly lower than room temperature (see Fig. 5(g)). Using this method, Zhang et al. demonstrated the O-T phase coexistence in $\mathrm{KNNS}_{0.04}-0.04 \mathrm{BNZ}$ and $\mathrm{KNNS}_{0.045^{-}}$ $0.0375 \mathrm{BNH}$ ceramics, ${ }^{68,69}$ whose phase structure was previously identified as the NPB with R-T phase coexistence. ${ }^{45,54}$ This difference can be ascribed to several reasons. Firstly, their $T_{\mathrm{R}-\mathrm{O}}$ values were actually also diffused, and $T_{\mathrm{O}-\mathrm{T}}$ values were slightly higher than room temperature (see Fig. 5(h) and (j)), indicating a further need for additives. Furthermore, their unpoled XRD patterns exhibited two comparable peaks in $\{200\}_{\mathrm{pc}}$ diffraction like that of KNNS-SZ- $x$ BAZ ceramics $(x=0.025)$ (see Fig. 5(i) and $(\mathrm{k})$ ), further proving the need of more additives. As expected, they recently observed an $\mathrm{R}-\mathrm{O}-\mathrm{T}$ multi-phase coexistence in $\mathrm{KNNS}_{0.04}-x \mathrm{BNH}(x=0.04-0.047)$ ceramics that contained a higher content of BNH. $\mathrm{KNNS}_{0.04}-x \mathrm{BNH}(x=0.04-0.047)$ ceramics possessed $d_{33}$ values as high as 450-540 $\mathrm{pC} \mathrm{N}^{-1}$ and exhibited similar $\{200\}_{\mathrm{pc}}$ diffraction peaks to those of KNNSSZ- $x$ BAZ $(x=0.03-0.035)$ (see Fig. $5(\mathrm{n})) .{ }^{72}$ More importantly, the corresponding $\varepsilon^{\prime \prime}-T$ curve also exhibited the almost converged $T_{\mathrm{R}-\mathrm{O}}$ and $T_{\mathrm{O}-\mathrm{T}}$, while $T_{\mathrm{R}-\mathrm{O}}$ was too weak to be recognized in the $\varepsilon^{\prime}-T$ curve (see Fig. $5(\mathrm{l})$ and $(\mathrm{m})$ ), which was the reason why they inferred an $\mathrm{R}-\mathrm{O}-\mathrm{T}$ multi-phase coexistence at room temperature. ${ }^{72}$ In the same way, they also observed the $\mathrm{R}-\mathrm{O}-\mathrm{T}$ multi-phase coexistence in $(0.994-x)\left(\mathrm{K}_{0.40} \mathrm{Na}_{0.60}\right)\left(\mathrm{Nb}_{0.955} \mathrm{Sb}_{0.045}\right) \mathrm{O}_{3}-$ $0.006 \mathrm{BiFeO}_{3}-x\left(\mathrm{Bi}_{0.50} \mathrm{Na}_{0.50}\right) \mathrm{ZrO}_{3}(x=0.01-0.04) .{ }^{73}$ Secondly, the diffused $T_{\mathrm{R}-\mathrm{O}}$ may also manifest the existence of the $\mathrm{R}$ phase. As we know, the phase transitions of piezoceramics are gradual, not as rapid as in single crystals due to the grain size distribution and intragranular stresses. ${ }^{74}$ Thus, the multi-phase coexistence may exist over a wide temperature range. Actually, the Rietveld refinements showed that the lattice parameters of the $\mathrm{R}$ phase at the NPB were highly close to those of a cubic (C) phase. ${ }^{75-77}$ In other words, the R phase may be distorted to some degree, which made it difficult to clearly discern. Therefore, the researchers may possibly exclude it only from $\varepsilon_{\mathrm{r}}-T$ (also $\varepsilon^{\prime}-T$ ) curves. Finally, the exact structure and performance of a material were strongly dependent on the detailed fabrication process, ${ }^{78,79}$ which may also contribute to the difference. Therefore, the most essential difference between O-T phase boundary and NPB is whether $T_{\mathrm{R}-\mathrm{O}}$ has shifted (or diffused) to room temperature or not. Since $T_{\mathrm{R}-\mathrm{O}}$ usually became diffused after the addition of additives, 
one should use more precise tools to collect its evolution, such as $\varepsilon^{\prime \prime}-T$ curves of poled samples. However the exact phase structure at NPB should be further identified by other tools, which will be soon discussed in Section 2.1.

\section{Structure}

For a piezoelectric material, its phase structure and microstructure (e.g., grain size, domain structure, and local structure) will directly affect its performance, including the most cared piezoelectric properties and temperature stability. ${ }^{80}$ In this section, the identification of phase structure and the observation of domain configuration and local structure in KNN-based ceramics with NPB will be systematically reviewed and discussed, focusing on expounding why we call it "NPB".

\subsection{Phase structure}

Since the NPB has been successfully constructed at or near room temperature, it is necessary to analyze the phase structure of the NPB due to the close relationship of phase structure and electrical properties. ${ }^{81}$ The current widely used tools to judge the phase structure of the NPB include X-ray diffraction (XRD) patterns, high-energy synchrotron radiation, neutron diffraction, $\varepsilon_{\mathrm{r}}-T$ (also $\varepsilon^{\prime}-T$ and $\varepsilon^{\prime \prime}-T$ ) curves, Raman spectra, and highresolution transmission electron microscopy (HR-TEM). ${ }^{14}$ Due to the similar lattice parameters of different phases in multi-phase coexistence, it is difficult to directly distinguish the exact phase structure by normal XRD patterns. Therefore, the researchers mainly combined XRD patterns and other tools (e.g., $\varepsilon_{\mathrm{r}}-T$ curves, in situ temperature-dependent Raman spectra) to judge the phase structure of the NPB. ${ }^{28,31,82,83}$ For example, most researchers identified the phase structure of the NPB by simultaneously considering the composition-dependent XRD patterns and $\varepsilon_{\mathrm{r}}-T$ curves. ${ }^{67-69,84,85}$ The composition-dependent XRD could initially reflect the phase structure of KNN-based ceramics, while the variations of $T_{\mathrm{R}-\mathrm{O}}$ and $T_{\mathrm{O}-\mathrm{T}}$ values extracted from $\varepsilon_{\mathrm{r}}-T$ curves would further provide more information of phase structure at room temperature. Thus, the phase structure of the NPB was qualitatively identified. But however, the abnormal dielectric peak of $T_{\mathrm{R}-\mathrm{O}}$ is not as clear as that of $T_{\mathrm{O}-\mathrm{T}}$, as discussed in Fig. 5. Particularly, the addition of additives will further suppress the abnormal dielectric peak of $T_{\mathrm{R}-\mathrm{O}}$ and make it difficult to identify. ${ }^{67-69,84,85}$ Therefore, judging the phase structure from $\varepsilon_{\mathrm{r}}-T$ curves needs to be more cautious.

Here, we mainly introduced the application of TEM for identifying the phase structure of the NPB due to the high resolution and accuracy of TEM, particularly the mode of convergent beam electron diffraction (CBED). ${ }^{23,25,28,86-88}$ Due to the intrinsic symmetry of the space group, $\mathrm{R}, \mathrm{O}$, and $\mathrm{T}$ phases will show their own unique CBED patterns like the fingerprints of humans, by which the phase structure of the NPB can be clearly identified. Table 3 lists the detailed symmetry elements of R, O, and T phases. Fig. 6 shows the theoretical simulations

Table 3 The detailed symmetry elements of T, O, and R phases. The symbols "4" and "3" mean 4-fold and 3-fold rotation; " $m$ " means a mirror plane. Reproduced from ref. 23 with permission from the Royal Society of Chemistry, Copyright 2017

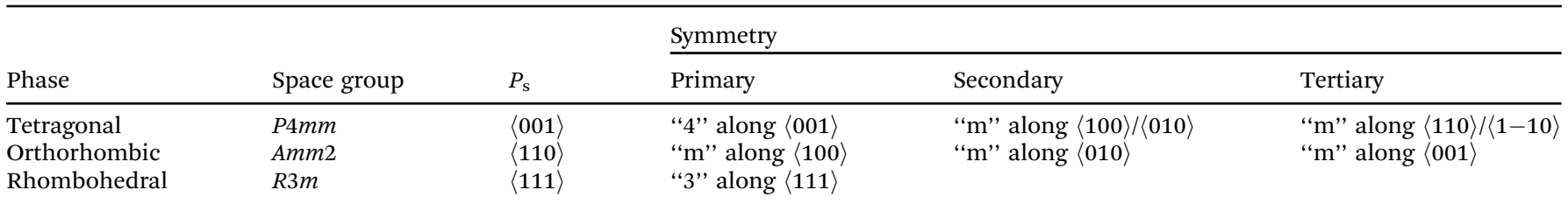

\begin{tabular}{|c|c|c|c|c|}
\hline \multirow{2}{*}{ Structural mode } & \multirow{2}{*}{$\begin{array}{c}\text { Space group \& } \\
\text { Lattice parameters }\end{array}$} & \multicolumn{3}{|c|}{ Pseudocubic zone axis } \\
\hline & & $\{001\}$ & $\{110\}$ & $\{111\}$ \\
\hline & $\begin{array}{l}\text { Tetragonal } \\
(\boldsymbol{P 4 m m}) \\
a=b \neq c \\
\alpha=\beta=\gamma=90^{\circ}\end{array}$ & $\begin{array}{llll}0 & 9 & 0 & 0 \\
0 & \phi & 0 & 0 \\
0 & 0 & 0 & \sigma\end{array}$ & $\begin{array}{l}89 \\
80 \\
80 \\
0\end{array}$ & \\
\hline & $\begin{array}{l}\text { Orthorhombic } \\
\left(\begin{array}{l}\text { Amm } 2) \\
a \neq b \neq c \\
\alpha=\beta=\gamma=90^{\circ}\end{array}\right.\end{array}$ & & & \\
\hline & $\begin{array}{l}\text { Rhombohedral } \\
\text { (R3m) } \\
a=b=c \\
\alpha=\beta=\gamma \neq 90^{\circ}\end{array}$ & a. & & \\
\hline
\end{tabular}

Fig. 6 Simulations of CBED patterns of $\mathrm{R}, \mathrm{O}$ and $\mathrm{T}$ phases along $\langle 001\rangle,\langle 110\rangle$ and $\langle 111\rangle$ zone axes. The red lines indicate the mirror planes. 


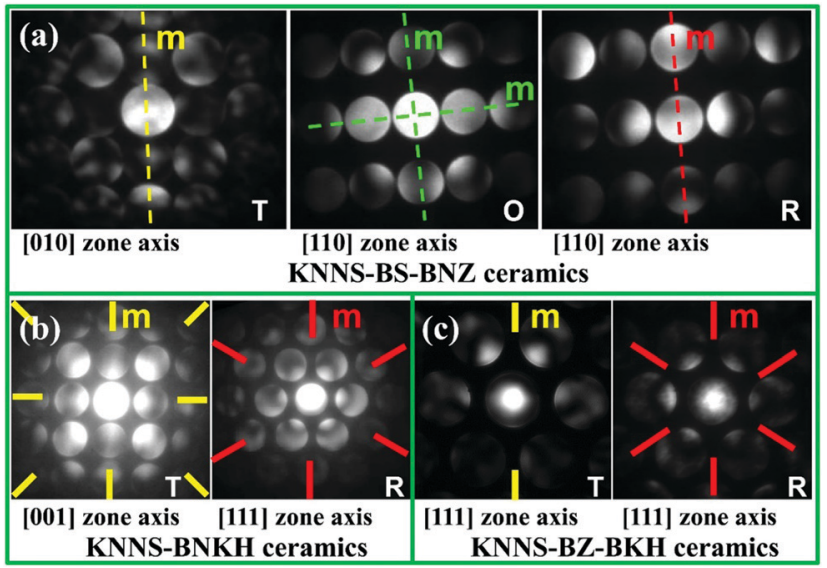

Fig. 7 Convergent beam electron diffraction (CBED) patterns of reported KNN-based ceramics with NPB. (a) $(1-x-y) \mathrm{K}_{0.5} \mathrm{Na}_{0.5} \mathrm{Nb}_{0.96} \mathrm{Sb}_{0.04} \mathrm{O}_{3}-$ $x \mathrm{BaSnO}_{3}-y \mathrm{Bi}_{0.5} \mathrm{Na}_{0.5} \mathrm{ZrO}_{3}$ (KNNS-BS-BNZ, $x=0.01$ and $y=0.035$ ) ceramics, (b) $(1-x) \mathrm{K}_{1-y} \mathrm{Na}_{y} \mathrm{Nb}_{1-z} \mathrm{Sb}_{z} \mathrm{O}_{3}-x \mathrm{Bi}_{0.5}\left(\mathrm{Na}_{1-w} \mathrm{~K}_{w}\right)_{0.5} \mathrm{HfO}_{3}$ (KNNS-BNKH, $x=0.035$, $y=0.52, z=0.05$ and $w=0.18)$ ceramics, and (c) $(1-x-y)$ $\mathrm{K}_{1-w} \mathrm{Na}_{w} \mathrm{Nb}_{1-z} \mathrm{Sb}_{z} \mathrm{O}_{3}-y \mathrm{BaZrO}_{3}-x \mathrm{Bi}_{0.5} \mathrm{~K}_{0.5} \mathrm{HfO}_{3}$ (KNNS-BZ-BKH, $x=0.03$, $y=0.02, z=0.035, w=0.4$ ) ceramics. " $m$ " represents the mirror plane. (a) Reproduced from ref. 28 with permission from the American Chemical Society, Copyright 2016. (b) Reproduced reproduced from ref. 25 with permission from the Royal Society of Chemistry, Copyright 2017; (c) reproduced from ref. 23 with permission from Wiley-VCH, Copyright 2016.

of CBED patterns of $\mathrm{R}, \mathrm{O}$, and $\mathrm{T}$ phases. ${ }^{25}$ It should be mentioned that the $\mathrm{R}$ and $\mathrm{O}$ phases may exhibit highly similar CBED patterns along the $\langle 001\rangle$ zone axis with the same mirror along the $\langle 110\rangle$ zone axis. ${ }^{25}$ Therefore, it would be better to obtain CBED patterns along different zone axes. By means of CBED patterns, our group identified the phase structure of KNN-based ceramics with NPB, as shown in Fig. 7. For KNNS0.01BZ-0.035BNZ ceramics, one mirror along $\langle 001\rangle$ with the beam incidence along the $\langle 010\rangle$ zone axis suggested the existence of $\mathrm{T}$ phase, two perpendicular mirrors with beam incidence along the $\langle 110\rangle$ zone axis indicated an Amm2 symmetry (i.e., O phase), and one mirror along $\langle 1-10\rangle$ with beam incidence along $\langle 110\rangle$ pointed to an R phase (see Fig. 7(a)). ${ }^{28}$ Thus, CBED patterns clearly revealed the phase structure of KNNS-0.01BZ-0.035BNZ ceramics with NPB to be an R-O-T multi-phase coexistence. Subsequently, our group also demonstrated the phase structure of the NPB in KNNS-0.035BNKH and KNNS-0.02BZ-0.03BKH ceramics to be an $\mathrm{R}-\mathrm{T}$ phase coexistence in the same way (see Fig. 7(b) and (c)). ${ }^{23,25}$

In addition to CBED patterns, the scanning transmission electron microscopy with high angle annular dark field (STEM HAADF) images can also clearly identify the phase structure of KNN-based ceramics with NPB. As we know, the local symmetries of KNN-based ceramics (e.g., R, O, T phases) arise from the electric dipole formed by the relative displacement between the centers of negative and positive ions. ${ }^{1}$ Thus, for KNN-based ceramics, the displacement of the center $\mathrm{Nb}^{5+}$ cation with respect to the corner $\mathrm{Na}^{+} / \mathrm{K}^{+}$cation $\left(\delta_{\mathrm{Nb}-\mathrm{Na} / \mathrm{K}}\right)$ can be used to reflect the local symmetry. Our group firstly used STEM HAADF images to detect the phase structure of KNNS-BF-BNZ ceramics, as shown in Fig. $8 .^{26}$ Since the B site atoms $(\mathrm{Nb})$ were much heavier than the A site atoms $(\mathrm{K} / \mathrm{Na})$, the brighter spots in Fig. 8(a) were center atom columns that represented $\mathrm{B}$ site atoms $(\mathrm{Nb})$. Then, the peak finder method found two displacement directions of the center atoms with respect to the corners (see Fig. 8(b1)). The enlarged areas showed that one is along $(100)_{\mathrm{pc}}$, while the other is along $(111)_{\mathrm{pc}}$ (see Fig. 8(b2) and (b3)), respectively consistent with the theoretical simulations of $\mathrm{T}$ and $\mathrm{R}$ unit cells with the center atom displacements along $(100)_{\mathrm{pc}}$ and $(111)_{\mathrm{pc}}$ (see Fig. 8(c) and (d)). Therefore, the STEM HAADF images can clearly reveal the local symmetry of KNN-based ceramics with NPB.

By using the same method, our group recently clearly revealed the local symmetry of KNNS-BNKZ- $x$ AS-Fe $(x=1.6 \%)$ ceramics possessing an ultra-high $d_{33}$ value of $650 \pm 20 \mathrm{pC} \mathrm{N}^{-1},{ }^{24}$ as shown in Fig. 9. Since the nanodomains of KNNS-BNKZ- $x$ ASFe $(x=1.6 \%)$ ceramics reduced to the limitation of CBED patterns, the STEM HAADF method is very suited for detecting the local symmetry due to the high resolution. Fig. 9(a) shows the STEM
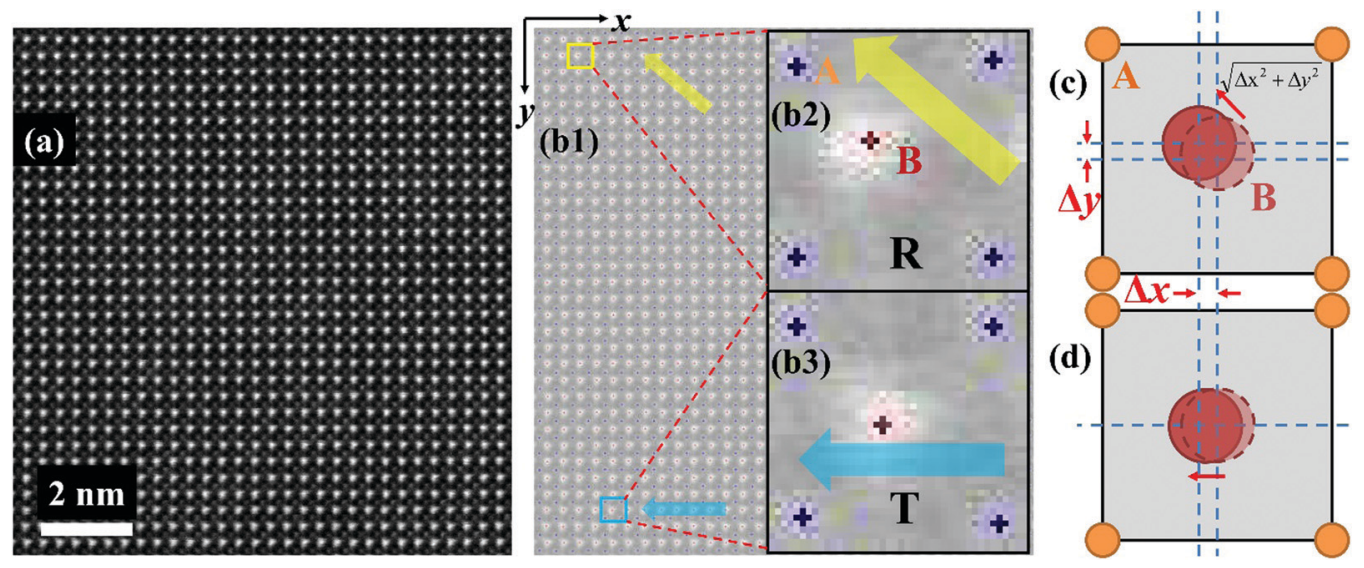

Fig. 8 Local symmetry of $(1-x-y) \mathrm{K}_{1-w} \mathrm{Na}_{w} \mathrm{Nb}_{1-z} \mathrm{Sb}_{z} \mathrm{O}_{3}-x \mathrm{BiFeO}_{3}-y \mathrm{Bi}_{0.5} \mathrm{Na}_{0.5} \mathrm{ZrO}_{3}(\mathrm{KNNS}-\mathrm{BF}-\mathrm{BNZ}, x=0.006, y=0.03, z=0.045$, and $w=0.6)$ ceramics. (a) STEM HAADF lattice image at the domain boundary. (b1) Peak finding of (a). (b2 and b3) Enlarged view of the areas within the yellow and blue boxes. (c) Schematic diagram showing $\mathrm{T}$ symmetry with $\mathrm{B}(\mathrm{Nb} / \mathrm{Sb} / \mathrm{Fe} / \mathrm{Zr}$ ) atom displacement $\Delta x$ along pseudo-cubic axes with respect to $\mathrm{A}$ ( $\mathrm{Na} / \mathrm{K} / \mathrm{Bi}$ ) atoms. (d) Schematic diagram showing R symmetry with B atom displacement along the cube diagonal $\left(\sqrt{\Delta x^{2}+\Delta y^{2}}\right)$, which can be decomposed into displacements along $x$ and $y$ axes. (a-d) Reproduced from ref. 26 with permission from the American Chemical Society, Copyright 2016. 


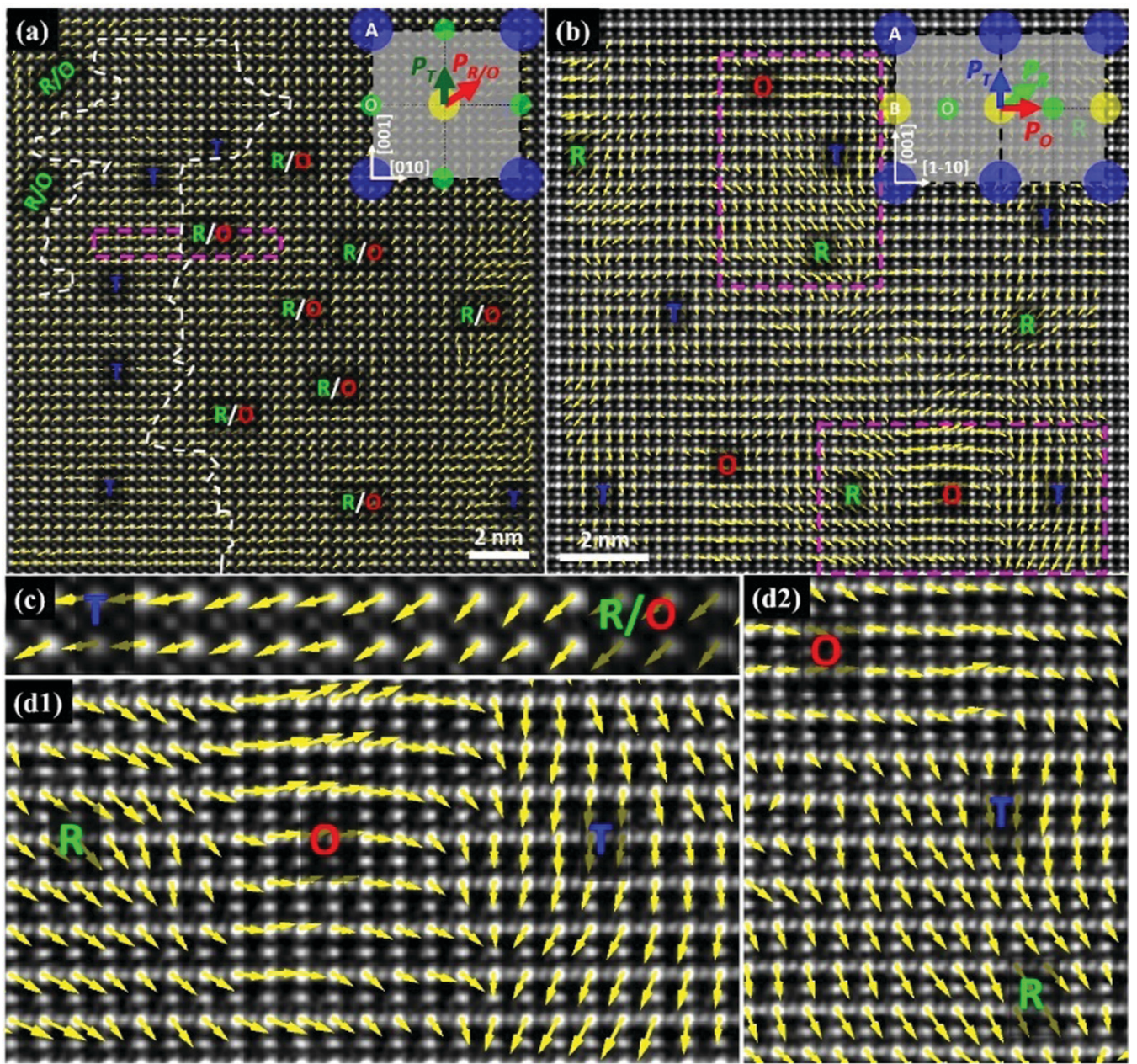

Fig. 9 Local symmetry and polarization mapping inside nanodomains of KNNS-BNKZ-AS-Fe ceramics. Atomically resolved STEM HAADF images along (a) $\langle 100\rangle$ and (b) $\langle 110\rangle$ zone axes. The corresponding insets show the schematic project of the $\mathrm{ABO}_{3}$ unit cell along $\langle 100\rangle$ and $\langle 110\rangle$ zone axes. When viewing the unit along the $\langle 100\rangle$ zone axis, the $T$ phase exhibits the polarization vector along $(001)_{p c}$, while both $R$ and $O$ phases display similar polarization vectors along $(110)_{p c}$. When viewing the unit along the $\langle 110\rangle$ zone axis, the T phase exhibits the polarization vector along $(001)_{p c}$, the $O$ phase

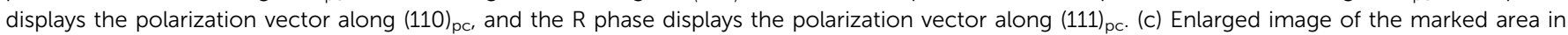
(a) showing the transition of polarization vectors from T to O/R. (d1 and d2) Enlarged images of the marked areas in (b) showing the transition of polarization vectors from R to O to T. (a-d2) Reproduced from ref. 24 with permission from the American Chemical Society, Copyright 2019.

HAADF image along the $\langle 100\rangle$ zone axis, in which the atom displacement vectors from the center $\mathrm{Nb}^{5+}$ cations to the corner $\mathrm{Na}^{+} / \mathrm{K}^{+}$cations $\left(\delta_{\mathrm{Nb}-\mathrm{Na} / \mathrm{K}}\right)$ are indicated by the yellow arrows. The $2 \mathrm{D}$ Gaussian peak fitting results clearly demonstrated the evidence of $\mathrm{T}$ phase, but could not directly distinguish the $\mathrm{R}$ and $\mathrm{O}$ phases because of their similar projections on the (100) plane, as schematically shown in the inset of Fig. 9(a). Subsequently, the two-dimensional (2D) Gaussian peak fitting for the STEM HAADF image along the $\langle 110\rangle$ zone axis directly observed the coexistence of R, O, and T phases (see Fig. 9(b)). Thus, the three phases coexisted within the scale of several nano-sized regions, forming a static slush nano-polar state. The detailed transformations of polarization vectors among different phases are enlarged and shown in Fig. 9(c), (d1) and (d2). It can be seen that the transition of polarization vectors was gradual, which was believed to reduce the polarization anisotropy and result in the easy polarization rotation, benefiting the piezoelectric properties. ${ }^{24}$
According to the reported references, two kinds of phase structures were widely reported in $\mathrm{KNN}$-based ceramics with $\mathrm{NPB}$, that is, $\mathrm{R}-\mathrm{T}$ and $\mathrm{R}-\mathrm{O}-\mathrm{T}$ phase coexistence. ${ }^{23,26,28,44,66}$ It is not surprising to find the evidence of the $\mathrm{T}$ phase at the NPB because $T_{\mathrm{O}-\mathrm{T}}$ is close to room temperature. The reported CBED patterns and STEM HAADF images also demonstrated the existence of the $\mathrm{R}$ phase. ${ }^{23,26,28,44,66}$ Therefore, the existence of the $\mathrm{O}$ phase or not was strongly dependent on the specific composition design. Indeed, increasing $T_{\mathrm{R}-\mathrm{O}}$ and decreasing $T_{\mathrm{O}-\mathrm{T}}$ will inevitably reduce the $\mathrm{O}$ phase zone, resulting in the reduced content of the $O$ phase. On the other hand, the different additives will affect $T_{\mathrm{R}-\mathrm{O}}$ and $T_{\mathrm{O}-\mathrm{T}}$ at different levels, as discussed in Section 1.2, which is the main reason why different phase structures were observed in different KNN-based ceramics with NPB. Therefore, extensive research on identifying the phase structure of the NPB is still needed, particularly finding a universal method to effectively detect the phase structure (e.g., O phase). 
The multi-phase coexistence at the NPB provided the primary basis for acquiring high piezoelectric properties. On the one hand, the multi-phase coexistence at the NPB will provide more possible polarization variants; on the other hand, the low polarization anisotropy of multi-phase coexistence can guarantee an easy polarization rotation, benefiting the macro piezoelectric properties. ${ }^{3,89-92}$ The detailed discussions about the effects of NPB's phase structure on the performance can be found in Section 3.1.3.

\subsection{Domain structure}

An area with the same spontaneous polarization direction is called a domain, and the boundary separating two domains is termed a domain wall. ${ }^{1,12}$ The domain structure plays an important role in the electrical properties and temperature stability of piezoelectric ceramics as an extrinsic contribution. ${ }^{93-96}$ Therefore, it is necessary to analyze the domain structure of KNN-based ceramics with NPB. Currently, TEM, piezoresponse force microscopy (PFM), and chemical etching are used to observe the domain structure of a piezoceramic. ${ }^{93-98}$ Fig. 9 shows the representative domain structure of $\mathrm{KNN}$-based ceramics with different phase boundaries at room temperature.

Pristine $\mathrm{K}_{0.5} \mathrm{Na}_{0.5} \mathrm{NbO}_{3}$ ceramics, which possess a pure $\mathrm{O}$ phase at room temperature, display regular domain structures with the shapes of "herringbone" and "watermark" (see Fig. 10(a)). 98,99 The herringbone domains correspond to $90^{\circ}$ domains, while the watermark ones represent $180^{\circ}$ domains. ${ }^{98,99}$ These domains generally exhibit a size of micron-scale, that is, $0.5-1 \mu \mathrm{m}$ in width and 3-7 $\mu \mathrm{m}$ in length for $90^{\circ}$ domains, and $3-10 \mu \mathrm{m}$ in width and $20-40 \mu \mathrm{m}$ in length for $180^{\circ}$ domains. ${ }^{98,99}$ The observed regular domain structures in pure $\mathrm{KNN}$ ceramics were determined by the intrinsic symmetry of the $\mathrm{O}$ phase. The $\mathrm{O}$ phase possesses 12 possible polarization variants along $\{110\}_{\mathrm{pc}}$ (see Fig. $\left.10(\mathrm{~d})\right){ }^{100}$ Therefore, only $60^{\circ}, 120^{\circ}, 90^{\circ}$, and $180^{\circ}$ domains can be found in pure KNN ceramics regardless of other factors, such as defect engineering and intersections of these domains. ${ }^{98,101}$

After constructing the $\mathrm{O}-\mathrm{T}$ phase boundary or NPB at (or near) room temperature, the domain structures of KNN-based ceramics are significantly changed in both size and shape. Firstly, KNN-based ceramics with multi-phase coexistence show miniaturized domains with the average size less than $0.5 \mu \mathrm{m} .{ }^{62,68,102,103}$ Of particular importance is the fact that KNN-based ceramics with NPB even exhibit submicron and nanoscale domains. It was reported that the miniaturized domains were more easily prone to switching with respect to the large counterparts under an external stimulus (e.g., electric field and stress), benefiting the piezoelectric properties. ${ }^{93-96}$ Then, the regular domain structures are replaced by complex ones. KNN-based ceramics with $\mathrm{O}-\mathrm{T}$ phase boundary exhibit striped and curved domains with an average scale of $200 \mathrm{~nm}$ (see Fig. 10(b)). ${ }^{102}$ Particularly, KNNSLT ceramics exhibited striped microdomains with a scale of $200-300 \mathrm{~nm}$ that were comprised of alternative and hierarchical nanodomains with a scale of $20 \mathrm{~nm}$ and $50 \mathrm{~nm}$ (see Fig. 10(b)). ${ }^{102}$ The CBED patterns demonstrated these alternate nanodomains to possess an $\mathrm{O}$ phase or a $\mathrm{T}$ phase. ${ }^{102}$
However, the KNN-based ceramics with NPB exhibited more complicated domain configurations compared to the ones with $\mathrm{O}$ phase and $\mathrm{O}-\mathrm{T}$ phase boundary, showing the shapes of striped, curved, watermarks, short segments, tweed-like, and nanoscale (see Fig. 10(c)). The chemical etching method unveiled the substantial short "segment" domains and "watermark" domains in KNNS-0.0375BNKZ ceramics with an R-T phase coexistence (see Fig. 10(c1) and (c2)). ${ }^{104}$ The short segment domains were due to the phase coexistence and the existence of $180^{\circ}$ ferroelectric domain walls, while the watermark domains were related to the rhombohedral $180^{\circ}$ ferroelectric domain walls. ${ }^{104}$ KNNS$0.035 \mathrm{BNKH}$ ceramics with an $\mathrm{R}-\mathrm{T}$ phase coexistence exhibited striped domains with a scale of 50-200 $\mathrm{nm}$ that consisted of hierarchical nanodomains with a scale of 10-30 nm (see Fig. 10(c3)). ${ }^{25}$ Similar domain configurations were also reported in other piezoelectric materials with multi-phase coexistence and high performance, such as 50BZT-50BCT, MPB-featured $\mathrm{PZT}$, and relaxor-PbTiO ${ }_{3}$ ceramics as well as BNT-BT and relaxor- $\mathrm{PbTiO}_{3}$ single crystals. ${ }^{87,105-109}$ Both KNNS-0.05BZ$0.02 \mathrm{BNH}-\mathrm{MnO}_{2}$ and $\mathrm{KNNS}-\mathrm{SZ}-x \mathrm{BAZ}(x=0.035)$ ceramics displayed rectangular-ambulatory domain structures composed of striped and tweed-like nanodomains (see Fig. 10(c4)-(c7)). ${ }^{29,32}$ More importantly, KNNS-0.02BZ-0.03BKH and KNNS-0.006BF$0.03 \mathrm{BNZ}$ ceramics, which possessed an $\mathrm{R}-\mathrm{T}$ phase coexistence and an ultra-high piezoelectricity (e.g., $d_{33}=550-570 \mathrm{pC} \mathrm{N}^{-1}$ ), universally displayed the ultra-fine domain with a scale of $1.8 \mathrm{~nm}$ (see Fig. 10(c8)-(c10)). ${ }^{23,26}$ Very recently, our group reported an ultra-high $d_{33}$ of $650 \pm 20 \mathrm{pC} \mathrm{N}^{-1}$ in KNNS-BNKZ- $x$ AS-Fe $(x=1.6 \%)$ ceramics that even displayed only ultra-thin nanodomains with unjudged size (see Fig. 10(c11)). Those ultra-thin nanodomains existed in a slush nano-polar state, resulting in the strong relaxor behavior. ${ }^{24}$

The complex domain configurations in KNN-based ceramics with NPB mainly originate from the multi-phase coexistence that possesses more possible polarization variants (see Fig. 10(e)). ${ }^{100}$ Furthermore, the polarization variants of different phases will interact with each other, resulting in complicated domain configurations. ${ }^{100}$ From the viewpoint of free energy, the domain size was also reduced as a consequence of the flattened energy profile in the multi-phase coexistence region. ${ }^{87}$ According to classical theory, $D \propto \sqrt{W_{\mathrm{DW}}}$, where $D$ is the domain size and $W_{\text {DW }}$ is the domain wall energy. ${ }^{87}$ The stable multi-phase coexistence indicates the low polarization anisotropy that will lead to the decrease of domain wall energy and consequently reduce the size of the domain. ${ }^{87}$ Subsequently, the miniaturized domains at the NPB were easily switched by applying an external electric field, promoting the macro performance of KNN-based ceramics. $^{93-96,110}$

\subsection{Local heterogeneity}

The local heterogeneity, which is also referred to as polar nanoregions (PNRs) in relaxor ferroelectrics, is largely discussed in lead-based piezoelectric materials (such as PMN-PT and PZNPT), ${ }^{81,91,111}$ but rarely reported in KNN-based ceramics. The NPB constructed by doping indispensable additives would inevitably destroy the long-range ordered lattice of KNN ceramics, 


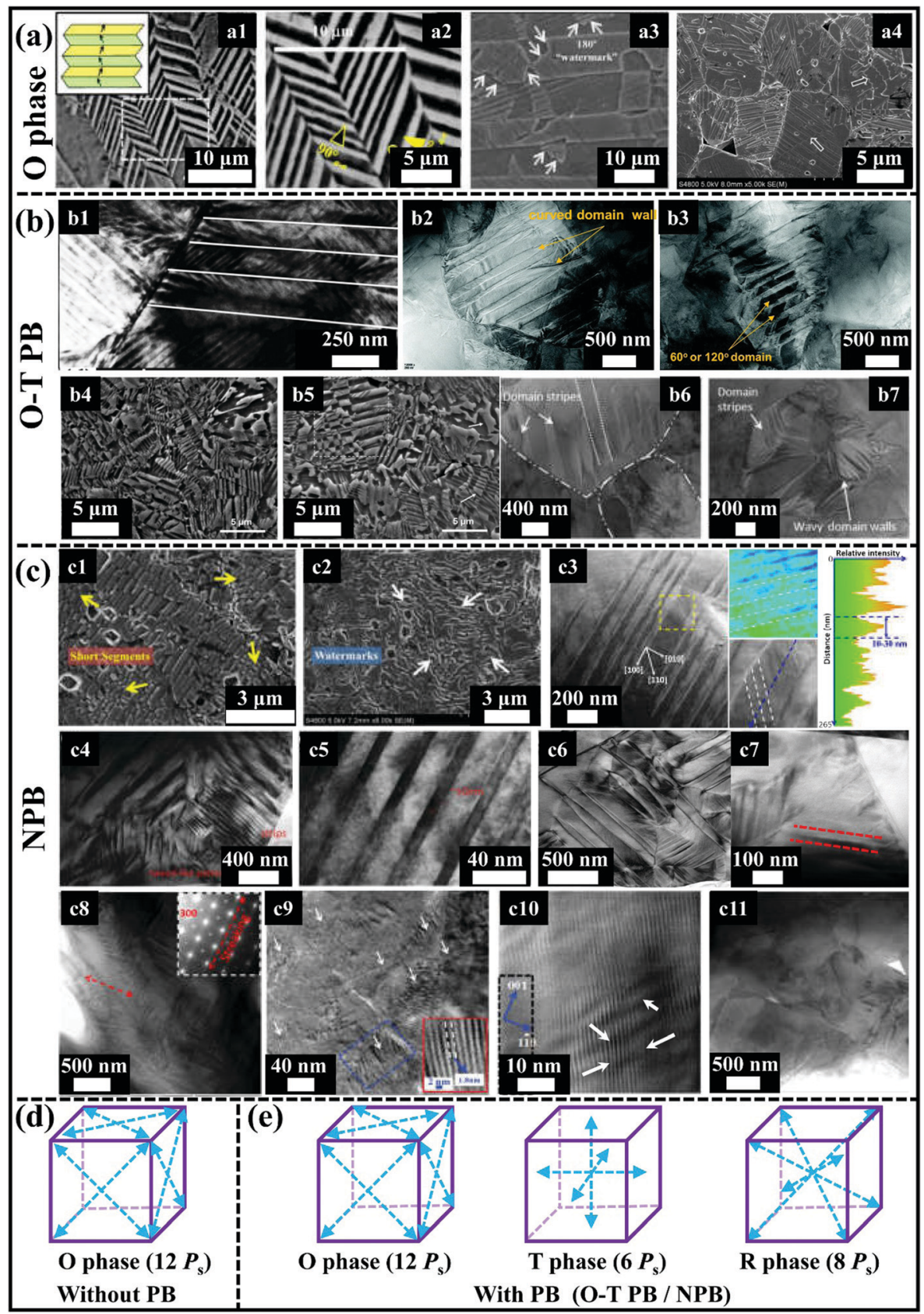

Fig. 10 Domain structure of KNN-based ceramics with different phase boundaries at room temperature. (a-c) Domain structures of KNN-based ceramics with different phase structures. (a1-a3) $\mathrm{K}_{0.5} \mathrm{Na}_{0.5} \mathrm{NbO}_{3}$ ceramics, (a4) $\mathrm{K}_{0.5} \mathrm{Na}_{0.5} \mathrm{NbO}_{3}$ ceramics, (b1) KNNSLT ceramics, (b2 and b3) KNNL0.065BZ-0.01BNT ceramics, (b4 and b5) KNNS-0.04BNZ ceramics, (b6 and b7) KNNSL-BNZ-BZ-MnO 2 ceramics, (c1 and c2) KNNS-0.0375BNKZ ceramics, (c3) KNNS-0.035BNKH ceramics, (c4 and c5) KNNS-0.05BZ-0.02BNH-MnO 2 ceramics, (c6 and c7) KNNS-SZ-xBAZ ( $x=0.035)$ ceramics, (c8) KNNS-0.006BF-0.03BNZ ceramics, (c9 and c10) KNNS-0.02BZ-0.03BKH ceramics, (c11) KNNS-BNKZ-xAS-Fe $(x=1.6 \%)$ ceramics. Schematic diagram of spontaneous polarization in KNN-based ceramics (a) without phase boundary and (e) with phase boundaries. (a1-a3) Reproduced from ref. 99 with permission from Elsevier, Copyright 2011; (a4) reproduced from ref. 98 with permission from the American Institute of Physics, Copyright 2013; (b1) reproduced from ref. 102 with permission from the American Institute of Physics, Copyright 2011; (b2 and b3) reproduced from ref. 103 with permission from the Royal Society of Chemistry, Copyright 2018; (b4 and b5) reproduced from ref. 68 with permission from the American Institute of Physics, Copyright 2018; (b6 and b7) reproduced from ref. 62 with permission from the Royal Society of Chemistry, Copyright 2018; (c1 and c2) reproduced from ref. 104 with permission from the American Chemical Society, Copyright 2016; (c3) reproduced from ref. 25 with permission from the Royal Society of Chemistry, Copyright 2017; (c4 and c5) reproduced from ref. 29 with permission from the Royal Society of Chemistry, Copyright 2018; (c6 and c7) reproduced from ref. 32 with permission from the Royal Society of Chemistry, Copyright 2019; (c8) reproduced from ref. 26 with permission from the American Chemical Society, Copyright 2016; (c9 and c10) reproduced from ref. 23 with permission from Wiley-VCH, Copyright 2016; (c11) reproduced from ref. 24 with permission from the American Chemical Society, Copyright 2019. 

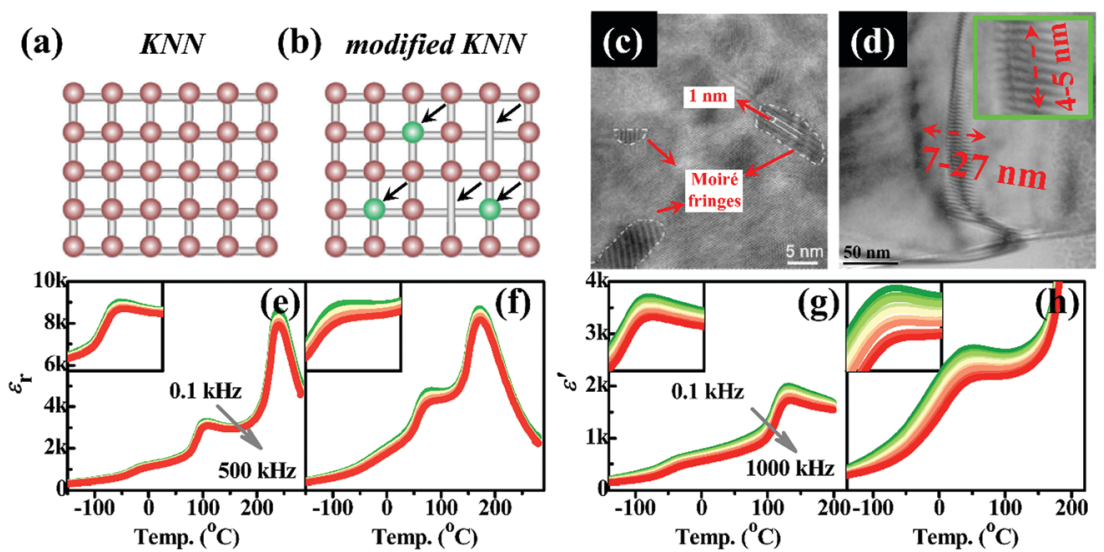

Fig. 11 TEM images of local heterogeneity of KNN-based ceramics with NPB. Simulated diagram of lattice distribution of (a) pure KNN ceramics and (b) KNN-based ceramics modified with additives. The black arrows indicate the location of local heterogeneity. TEM images of local heterogeneity in (c) $\mathrm{KNNSb}_{x}-\mathrm{BZ}-\mathrm{BNH}-\mathrm{MnO}_{2}(x=0.025)$ ceramics and (d) KNNS-SZ- $x \mathrm{BAZ}(x=0.035)$ ceramics. $\varepsilon_{\mathrm{r}}-\mathrm{T}$ curves of $\mathrm{KNNSb}_{x}-\mathrm{BZ}-\mathrm{BNH}-\mathrm{MnO}_{2}$ ceramics with $x=0$ (e) and $x=0.025$ (f). Temperature-dependent real part of permittivity $\left(\varepsilon^{\prime}-T\right)$ of KNNS-SZ-xBAZ ceramics with $x=0$ (g) and $x=0.035(\mathrm{~h})$. The inset

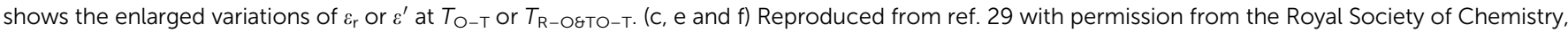
Copyright 2018; (d, g and h) reproduced from ref. 32 with permission from the Royal Society of Chemistry, Copyright 2019.

resulting in the occurrence of local heterogeneity, as simulated in Fig. 11(a) and (b). ${ }^{101} \mathrm{Li}$ et al. and our group observed the local heterogeneity in KNN-based ceramics with NPB by HR-TEM, ${ }^{29,32}$ as shown in Fig. 11(c) and (d). The local heterogeneity exhibited the scale of several nanometers. The local heterogeneity endows the KNN-based ceramics with improved degree of diffusion/relaxor, resulting in enhanced frequency dependence of permittivity when compared with those of the ones without being significantly modified by the additives. As shown in Fig. 11(e)-(h), $T_{\mathrm{O}-\mathrm{T}}$ of both $\mathrm{KNNS}_{x}-\mathrm{BZ}-\mathrm{BNH}-\mathrm{MnO}_{2}$ and KNNS-SZ- $x$ BAZ ceramics became diffused visibly after constructing the NPB. ${ }^{29,32}$ The local heterogeneity was believed to facilitate the domain switching and promote the piezoelectric properties. $^{81,91,111}$ To date, the investigations on the local heterogeneity of KNN-based ceramics with NPB are still rarely reported, which deserve further study in the future.

\subsection{Why it is called NPB}

Here, we emphatically explain the difference between NPB and previously reported phase boundaries (e.g., $\mathrm{R}-\mathrm{O}$ and $\mathrm{O}-\mathrm{T}$ phase boundary) and the reason why we call it "NPB". Firstly, the ways of constructing these phase boundaries are different. For the R-O or O-T phase boundary, we only need to shift $T_{\mathrm{R}-\mathrm{O}}$ or $T_{\mathrm{O}-\mathrm{T}}$ alone to or near room temperature, while the NPB requires the simultaneous shifting of $T_{\mathrm{R}-\mathrm{O}}$ and $T_{\mathrm{O}-\mathrm{T}}$ towards room temperature. The detailed explanation for the difference between NPB and O-T phase boundary can be found in Section 1.3. Secondly, the phase structure and domain configuration among these phase boundaries are significantly different. The $\mathrm{R}-\mathrm{O}$ or $\mathrm{O}-\mathrm{T}$ phase boundary possesses its corresponding multi-phase coexistence (e.g., $\mathrm{R}-\mathrm{O}$ or O-T phase coexistence), while the NPB can exhibit an R-T or an R-O-T multi-phase phase coexistence. Meanwhile, the NPB shows a more complicated domain structure and smaller domain size compared to the $\mathrm{R}-\mathrm{O}$ or $\mathrm{O}-\mathrm{T}$ phase boundary (see Fig. 10). Finally, the NPB exhibits significantly enhanced piezoelectric properties and improved temperature stability when compared with the $\mathrm{R}-\mathrm{O}$ or $\mathrm{O}-\mathrm{T}$ phase boundary (as will be discussed soon). Therefore, we call it "NPB", which presumptively represents $\mathrm{R}-\mathrm{T}$ and $\mathrm{R}-\mathrm{O}-\mathrm{T}$ phase boundaries. But however, one should keep in mind that the NPB still belongs to the PPB, which will inevitably exhibit temperature dependence of piezoelectric properties, as discussed in Section 1.3. Therefore, seeking a benign balance between piezoelectric properties and temperature stability has become one of the hottest topics in all lead-free piezoelectric materials.

\section{Performance and physical mechanisms}

\subsection{Piezoelectric properties}

Piezoelectric properties are the most important factors to evaluate the performance of a piezoceramic. .,2,5,6,9,10,19,22 $^{\text {Actually, }}$ the previous efforts were mainly put into the realization of high piezoelectric properties because of the relatively inferior piezoelectric properties in pristine KNN ceramics. In this part, we first summarize the piezoelectric properties at different phase boundaries, focusing on showing the effects of the NPB on the piezoelectric properties of KNN-based ceramics. Then, other approaches to further improve the piezoelectric properties at the NPB are introduced, such as slush nano-polar state, two-step sintering, controlling the phase ratio, and controlling the poling conditions. ${ }^{24,112-115}$ Finally, physical mechanisms underlying the piezoelectricity enhancement at the NPB are emphasized with the consideration of both intrinsic and extrinsic contributions.

3.1.1 Current piezoelectric properties at the NPB. Fig. 12(a) shows the piezoelectric properties of KNN-based ceramics with different phase boundaries. ${ }^{10,14} \mathrm{KNN}$-based ceramics with NPB generally exhibited much higher $d_{33}$ values compared to the ones with $\mathrm{R}-\mathrm{O}$ and $\mathrm{O}-\mathrm{T}$ phase boundaries, indicating that the NPB can further promote the piezoelectric properties of 

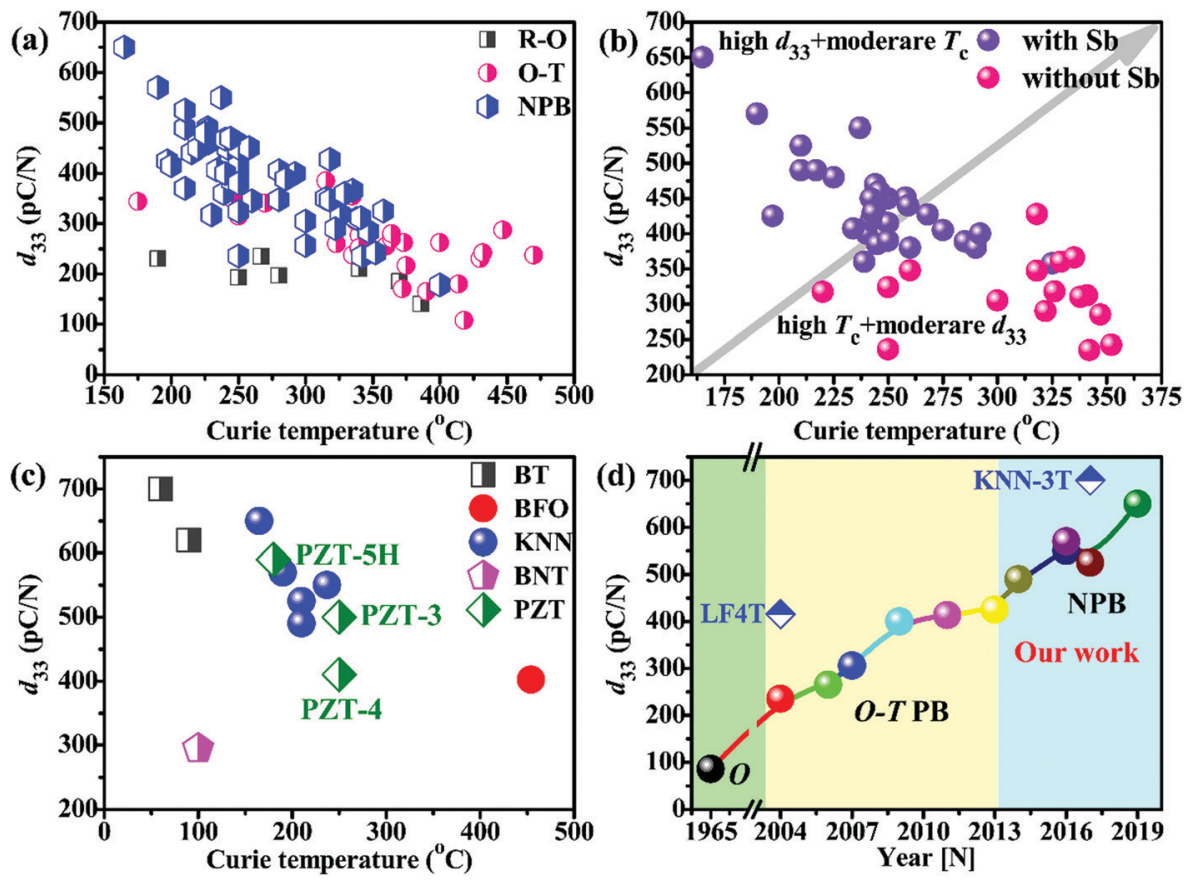

Fig. 12 Piezoelectric properties of non-textured KNN-based ceramics. (a) $d_{33}$ and $T_{c}$ of KNN-based ceramics with different phase boundaries. (b) $d_{33}$ and $T_{\mathrm{c}}$ of the NPB constructed with and without Sb. (c) State-of-the-art piezoelectric properties reported for BT-, KNN-, BFO-, and BNT-based lead-free piezoceramics and PZT-based ceramics. (d) Evolution of $d_{33}$ in KNN-based ceramics as a function of year. (d) Reproduced from ref. 23 with permission from Wiley-VCH, Copyright 2016.

KNN-based ceramics to a higher level. This is also one of the intrinsic differences between NPB and $\mathrm{R}-\mathrm{O} / \mathrm{O}-\mathrm{T}$ phase boundaries. Particularly, our group have successfully obtained ultra-high $d_{33}$ values of $490-650 \mathrm{pC} \mathrm{N}^{-1}$ in $\mathrm{KNN}$-based ceramics by constructing the NPB and by optimizing the composition design, which are the highest $d_{33}$ values reported so far in non-textured KNNbased ceramics. ${ }^{23-26,44}$

By further analyzing the piezoelectric properties at the NPB, we found that the composition design also significantly affected the piezoelectric properties of $\mathrm{KNN}$-based ceramics, particularly the addition of antimony (Sb) or not (see Fig. 12(b)). With the addition of $\mathrm{Sb}, \mathrm{KNN}-$ based ceramics with NPB can exhibit a combination of "high $d_{33}$ and moderate $T_{\mathrm{c}}$ ", 23-26,28,29,31,41-45,55,57,59,60,63-66,97,103,116-145 while the counterparts without Sb can show a combination of "high $T_{\mathrm{c}}$ and moderate $d_{33}$ ". ${ }^{27,62,146-165}$ Therefore, in addition to the construction of the phase boundary, the composition design should also be fully considered when pursuing high piezoelectric properties. In other words, the NPB can provide a high platform, while the composition design determines the maximum level of piezoelectric properties of KNN-based ceramics. The detailed physical mechanisms will be discussed later.

Here, we also summarize state-of-the-art piezoelectric properties of typical non-textured lead-free piezoelectric ceramics, such as BT-, KNN-, BNT-, and BFO-based ceramics (see Fig. 12(c)). Obviously, each one occupied a unique area of Fig. 12(c). BT-based ceramics showed a high $d_{33}$ of $620-700 \mathrm{pC} \mathrm{N}^{-1}$, but a $T_{\mathrm{c}}$ lower than $120{ }^{\circ} \mathrm{C}^{19,82,166}$ thus the location of BT-based ceramics was at the top left corner of Fig. 12(c). When improving the $d_{33}$ of BNT-based ceramics to a relatively high value (e.g., $200-300 \mathrm{pC} \mathrm{N}^{-1}$ ), a relatively low depolarization temperature $\left(T_{\mathrm{d}}\right)$ less than $200{ }^{\circ} \mathrm{C}$ was always observed, over which the net piezoelectric properties disappeared. ${ }^{167-169}$ Therefore, BNT-based ceramics mainly occupied the bottom left corner of Fig. 12(c). Quenched BFObased ceramics displayed both a large $d_{33}$ of $400 \mathrm{pC} \mathrm{N}^{-1}$ and a high $T_{\mathrm{c}}$ of $450 \mathrm{pC} \mathrm{N}^{-1}, 39$ occupying the right area of Fig. 12(c). However, the quenching process cannot guarantee good reproducibility, which needs to be further improved in the future. KNN-based ceramics with NPB located at the center of Fig. 12(c), showing a high $d_{33}$ and a moderate $T_{\mathrm{c}} \cdot{ }^{23-26,44}$ Thus, the performance of KNN-based ceramics with NPB was comparable to that of partly commercial PZT ceramics (e.g., PZT-3, PZT-4, and PZT-5H ceramics) (see Fig. 12(c)). Therefore, from the viewpoint of piezoelectric properties, KNN-based ceramics with NPB are the most promising candidates to replace the lead-based ones.

Finally, we conclude with the evolution of non-textured $\mathrm{KNN}$ based ceramics' $d_{33}$ values as a function of year (see Fig. 12(d)). An extremely slow increase of $d_{33}$ was observed before 2004 due to the absence of phase boundary engineering. In 2004, Satio et al. firstly achieved a large $d_{33}$ of $416 \mathrm{pC} \mathrm{N}^{-1}$ in textured LF4T ceramics with $\mathrm{O}-\mathrm{T}$ phase boundary. ${ }^{22}$ On the other hand, the corresponding non-textured $\mathrm{KNN}$-based ceramics exhibited a relatively low $d_{33}$ of less than $300 \mathrm{pC} \mathrm{N}^{-1} \cdot{ }^{22}$ After that, the major research concentrated on the construction of the $\mathrm{O}-\mathrm{T}$ phase boundary, resulting in the continuous increase of $d_{33}$. It was not until 2009 that Zuo et al. reported a $d_{33}$ of $400 \mathrm{pC} \mathrm{N}^{-1}$ in nontextured KNNSLT ceramics, ${ }^{40}$ which was merely comparable to that of textured LF4T ceramics. In 2013, our group firstly reported a high $d_{33}$ of $425 \mathrm{pC} \mathrm{N}^{-1}$ in KNNLS-BZ ceramics with 
an R-T phase coexistence. ${ }^{42}$ It was the first time that such a high $d_{33}$ value in non-textured KNN-based ceramics was reported at that time, which was superior to that of textured LF4T ceramics. Then, our group obtained a series of breakthroughs in $d_{33}$ values of KNN-based ceramics by constructing the NPB and optimizing the composition design, obtaining the $d_{33}$ values as high as 400-650 $\mathrm{pC} \mathrm{N}^{-1} \cdot{ }^{23-26,44}$ These $d_{33}$ values were much higher than those of KNN-based ceramics with an R-O or O-T phase boundary and even close to that of highly textured $\mathrm{KNN}-3 \mathrm{~T}$ ceramics. ${ }^{86}$ Therefore, a further increase in $d_{33}$ is highly expected by further optimizing the composition design of KNN-based ceramics with NPB.

3.1.2 Way to further improve the piezoelectric properties at the NPB

Slush nano-polar state. As discussed in Fig. 10 and 11, the miniaturized domains are prone to switching under an external stimulus (e.g., electric field and stress), and the PNRs could further promote the domain switching. Although the coexistence of long-range ordered ferroelectric domains and PNRs could realize high piezoelectric properties, the relatively high energy barrier between them still exists. ${ }^{24,32,81,91,170}$ Therefore, if one can construct a NPB full of ultra-fine domains (e.g., the PNRs occupy the whole sample) without changing the multi-phase coexistence, better piezoelectric properties are highly expected due to the lower energy barrier. Based on this hypothesis, our group designed $(0.96-x) \mathrm{K}_{0.48} \mathrm{Na}_{0.52} \mathrm{Nb}_{0.95} \mathrm{Sb}_{0.05} \mathrm{O}_{3}-0.04 \mathrm{Bi}_{0.5}\left(\mathrm{Na}_{0.82} \mathrm{~K}_{0.18}\right)_{0.5}-$ $\mathrm{ZrO}_{3}-x \mathrm{AgSbO}_{3}-0.4 \% \mathrm{Fe}_{2} \mathrm{O}_{3}$ (KNNS-BNKZ- $x$ AS-Fe) ceramics. ${ }^{24}$ The KNNS-BNKZ matrix could guarantee the NPB (i.e., multi-phase coexistence), ${ }^{44}$ and the addition of $\mathrm{AgSbO}_{3}$ and $\mathrm{Fe}_{2} \mathrm{O}_{3}$ could break the large ferroelectric domains into ultra-fine domains. The TEM image showed that the whole sample was occupied by ultra-fine domains, whose size was too small to be measured (see Fig. 10(c11)). The STEM HAADF image proved the coexistence of $\mathrm{R}, \mathrm{O}$, and $\mathrm{T}$ phases within the scale of several nano-sized regions, forming a static slush nano-polar state (see Fig. 9 and 13(a)). These slush nano-polar states endowed KNNS-BNKZ$x \mathrm{AS}-\mathrm{Fe}(x=1.6 \%)$ ceramics with distinct relaxor behavior, which was different from the one in the traditional relaxor ferroelectric (see Fig. 13(b) and (c)). Finally, we obtained an ultra-high $d_{33}$ value of $650 \pm 20 \mathrm{pC} \mathrm{N}^{-1}$ at $x=1.6 \%$ and high $d_{33}$ values $\left(>600 \mathrm{pC} \mathrm{N}^{-1}\right.$ ) over a relatively wide range of $x=1.5-$ $2.5 \%$, which are the highest values so far reported in nontextured KNN-based ceramics (see Fig. 13(d) and (e)). Therefore, the relaxor-featured NPB induced by the slush nano-polar state is an effective way to further promote the piezoelectricity of $\mathrm{KNN}$-based ceramics in the future.

Two-step sintering. Two-step sintering involves first sintering the samples at a high temperature for a short time and then keeping the samples at a relatively low temperature for a long time, as simulated in Fig. 14(a). The first sintering temperature $\left(T_{1}\right)$ could guarantee the formation of the required crystalline structure, while the second one $\left(T_{2}\right)$ could control the density and grain size. ${ }^{68,69,85,112}$ Two-step sintering was reported to effectively improve the piezoelectric properties and control the density and grain size of piezoceramics. ${ }^{68,69,85,112}$ Zheng et al. systematically studied the effects of two-step sintering on KNNS-0.04BKZS ceramics with NPB (see Fig. 14(b)-(e)). ${ }^{112}$ The results showed that enhanced $d_{33}$ values of $350-400 \mathrm{pC} \mathrm{N}^{-1}$ could be guaranteed over a wide $T_{2}$ range of $400-1050{ }^{\circ} \mathrm{C}$. Such a gap of $650{ }^{\circ} \mathrm{C}$ in $T_{2}$ was much wider than that of previous results by two-step sintering, ${ }^{171,172}$ which could significantly reduce the cost for practical applications. In addition, the validity of this method was also demonstrated by other groups. For example, Zhang et al. increased $d_{33}$ values of KNNLST ceramics from $310 \mathrm{pC} \mathrm{N}^{-1}$ to $455 \mathrm{pC} \mathrm{N}^{-1}$ by carefully optimizing the sintering temperature and time of the two-step sintering method. ${ }^{85}$ Therefore, two-step sintering could not only guarantee the piezoelectric properties at the NPB but also promote the step towards industrial applications of the NPB by reducing the cost.

Controlling the phase ratio. In the two-step fabrication firstly some components are pre-synthesized and then they are mixed with other raw materials. This method may alter the activation energy during the sintering process, resulting in the change of the crystalline structure. ${ }^{113}$ Using the two-step fabrication, (a)

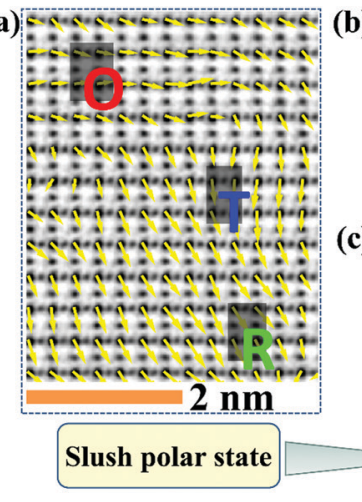

(b)

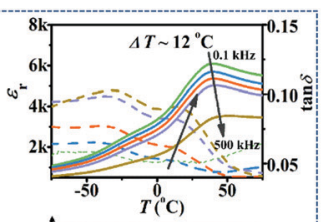

(c)

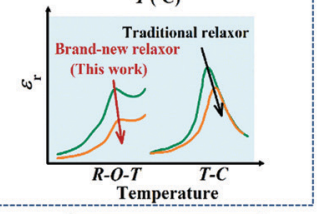

New relaxor (d)

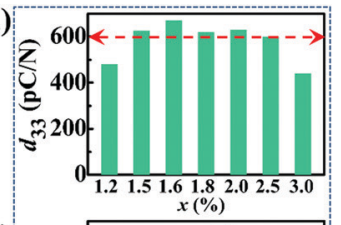

(e)

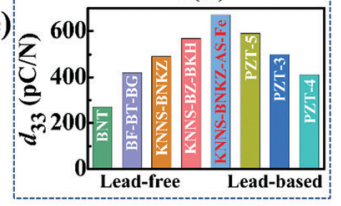

Ultra-high $d_{33}$

Fig. 13 (a) Enlarged STEM HAADF image and (b) $\varepsilon_{r}-T$ curves of KNNS-BNKZ-xAS-Fe $(x=1.6 \%)$ ceramics. (c) Comparison of the relaxor behavior of KNNS-BNKZ-xAS-Fe $(x=1.6 \%)$ ceramics and the traditional relaxor ferroelectric. (d) $d_{33}$ values as a function of $x$. (e) Comparison of $d_{33}$ values among representative lead-free and lead-based piezoceramics. (a-e) Reproduced from ref. 24 with permission from the American Chemical Society, Copyright 2019. 

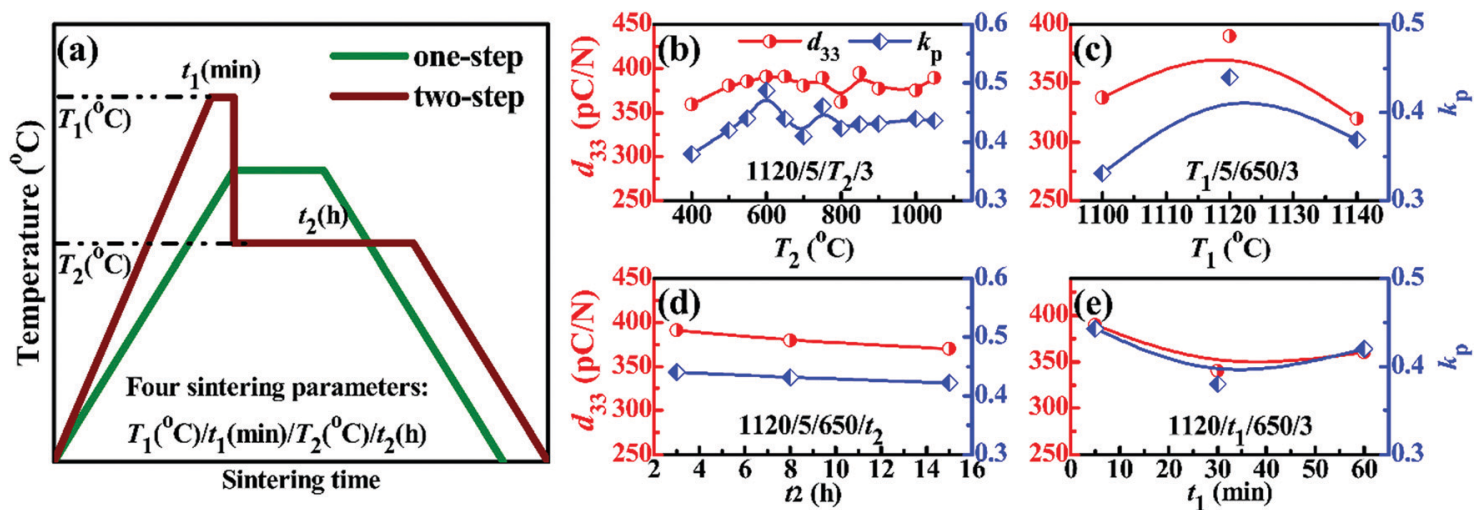

Fig. 14 (a) Schematic diagram of one-step sintering and two-step sintering methods. $d_{33}$ and $k_{\mathrm{p}}$ values of KNNS-0.04BKZS ceramics varying with (b) $T_{2}$, (c) $T_{1}$, (d) $t_{2}$, and (e) $t_{1}$. (a-e) Reproduced from ref. 112 with permission from the Royal Society of Chemistry, Copyright 2015.

we successfully fabricated NPB-phased $0.96 \mathrm{~K}_{0.5} \mathrm{Na}_{0.5} \mathrm{Nb}_{0.96} \mathrm{Sb}_{0.04} \mathrm{O}_{3}-$ $0.01 \mathrm{CaZrO}_{3}-0.03 \mathrm{Bi}_{0.5} \mathrm{Na}_{0.5} \mathrm{HfO}_{3}$ (KNNS-CZ-BNH) ceramics with different phase ratios. ${ }^{113}$ The $\mathrm{CaZrO}_{3}$ powder was firstly calcined at different temperatures for 2 hours, which was then mixed with other raw materials (see Fig. 15(a)). Subsequently, KNNS-CZ-BNZ ceramics containing different $\mathrm{CZ}$ powder (abbreviation: KNNSCZ-BNZ-calcination temperature) were prepared by using the conventional solid-state sintering method. The results showed that KNNS-CZ-BNZ- $900{ }^{\circ} \mathrm{C}$ ceramics possessed the highest piezoelectric properties (e.g., $d_{33}=445-455 \mathrm{pC} \mathrm{N}^{-1}$ ) among these different KNNS-CZ-BNZ ceramics (see Fig. 15(b)). The analysis suggested that both the phase ratio and the tetragonality $(c / a)$ were responsible for the difference in $d_{33}$ values. The higher the content of the $\mathrm{T}$ phase and the larger the $c / a$ ratio, the higher the $d_{33}$ values (see Fig. 15(c) and (d)). ${ }^{113}$ Therefore, controlling the phase ratio is another promising way to further improve the piezoelectric properties of KNN-based ceramics with NPB.
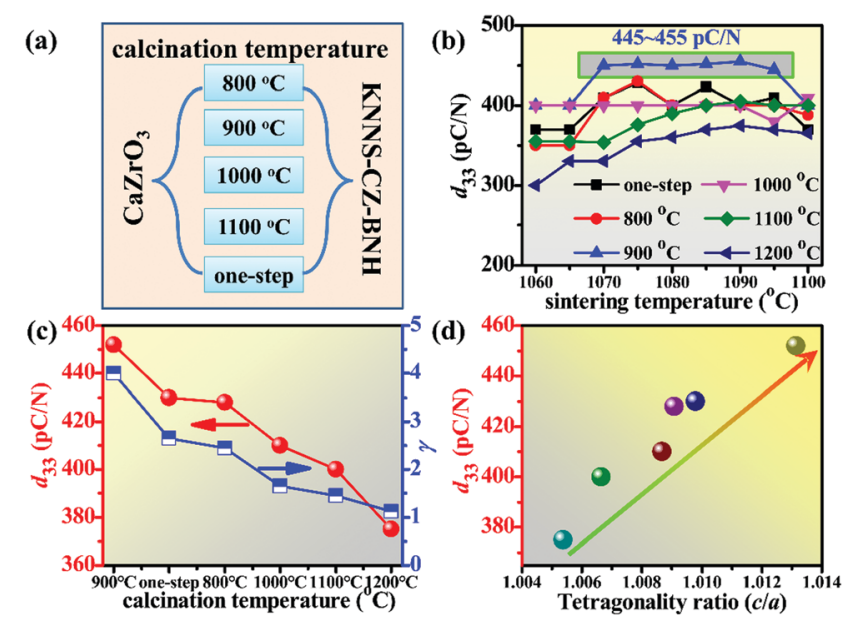

Fig. 15 (a) Schematic diagram showing the calcination temperature of $\mathrm{CaZrO}_{3}$ powder and related KNNS-CZ-BNH ceramics. (b) $d_{33}$ values of KNNS-CZ-BNH ceramics with different sintering temperatures. (c) $d_{33}$ values and the ratio of the $T$ phase to $R$ phase $\left(\gamma=T_{\text {content }} / R_{\text {content }}\right)$ of KNNS-CZ-BNH ceramics. (d) Relationship of $d_{33}$ and $\gamma$ of KNNS-CZ-BNH ceramics. (b-d) Reproduced from ref. 113 with permission from Elsevier, Copyright 2017.
Poling conditions. The poling conditions, including electric field, poling temperature $\left(T_{\mathrm{p}}\right)$, and type of electric field (direct current DC or alternative current AC), were also reported to greatly affect the piezoelectric properties of piezoelectric materials. ${ }^{114,115,173-179}$ The effects of electric field and $T_{\mathrm{p}}$ on the piezoelectric properties have been extensively investigated. ${ }^{173-177,179}$ The results showed that the optimized electric field and $T_{\mathrm{p}}$ could increase the piezoelectric properties to some degree. ${ }^{173-177,179}$ Recently, AC poling was reported to significantly improve the $d_{33}$ values of $\langle 001\rangle$-oriented rhombohedral relaxor-PT single crystals from 1096-1467 pC $\mathrm{N}^{-1}$ to 1371-1816 pC $\mathrm{N}^{-1}$, with the relative variations of $22.5-25.1 \% .{ }^{178}$ Inspired by their results, our group studied the effects of AC poling on the piezoelectric properties of KNN-based ceramics with NPB, as shown in Fig. 16(a)-(e). ${ }^{114,115}$ For both DC and AC poling, a threshold electric field was observed in KNNS-0.035BHNH ceramics, over which the saturated $d_{33}$ values could be obtained (see Fig. 16(c)). It should be noted that the threshold electric field of DC poling $\left(E_{\mathrm{DC}}\right)$ was much lower than that of AC poling $\left(E_{\mathrm{AC}}\right)$. The higher $E_{\mathrm{AC}}$ may be due to the short poling time and the difficulty in achieving the full domain reversal. ${ }^{114,115}$ More importantly, AC poling at a high electric field can obtain a higher $d_{33}$ than that of DC poling (see Fig. 16(c)). In addition, KNNS-0.035BNHN ceramics also exhibited frequency-independent AC poling behavior and comparable $T_{\mathrm{p}}$ dependence in both DC and AC poling (see Fig. 16(d) and (e)). And then, the relationship between DC/AC poling and phase boundaries was further investigated in KNN-based ceramics with different phase boundaries (see Fig. 16(f)-(j)). Once again, a higher $E_{\mathrm{AC}}$ was observed for all phase boundaries. $E_{\mathrm{DC}}$ values of all phase boundaries were close to their $E_{\mathrm{c}}$ values, as marked by the green area (see Fig. 16(f)-(j)). Though the higher threshold electric field (i.e., higher $E_{\mathrm{AC}}$ ), the AC poling still could achieve comparable or even higher $d_{33}$ values with respect to those of DC poling for all phase boundaries (see Fig. 16(f)-(j)). Therefore, AC poling may be a feasible way to further promote the piezoelectric properties of KNN-based ceramics with NPB.

Grain size. Controlling the grain size is also an effective method to improve the electrical properties of piezoceramics, 


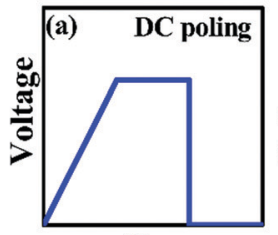

Time

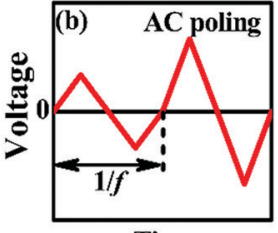

Time
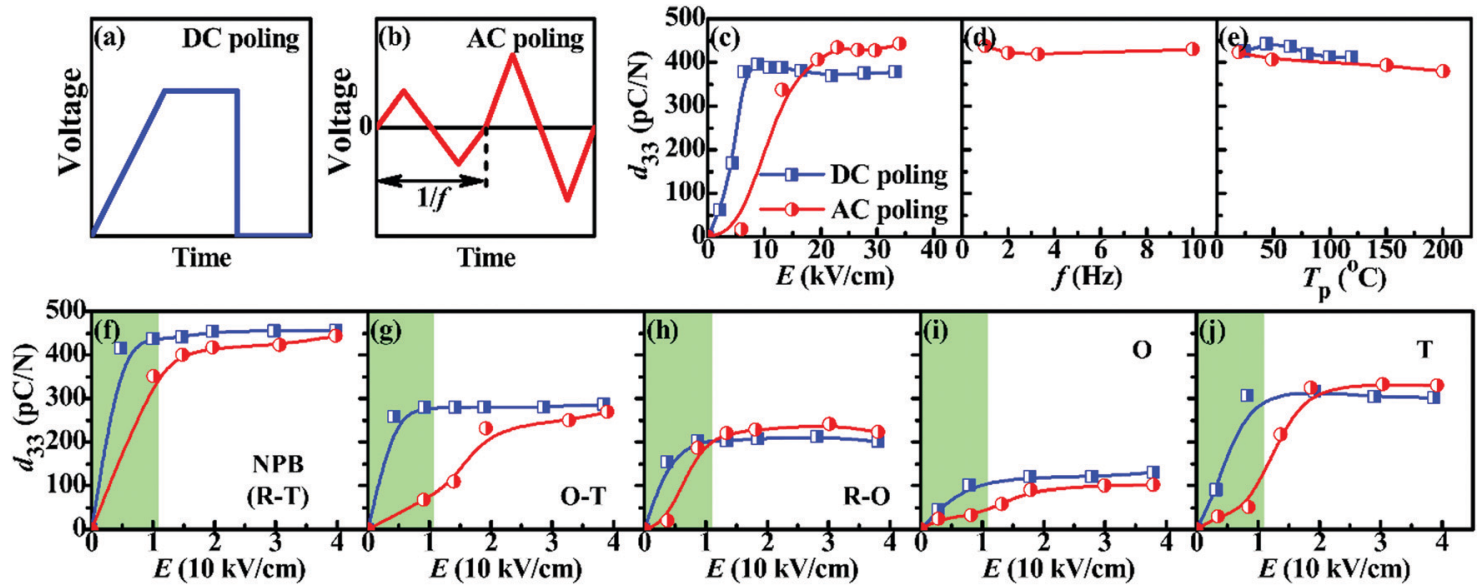

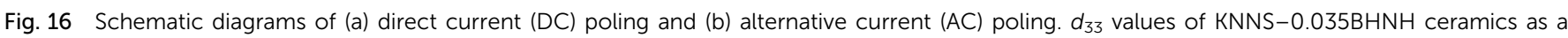

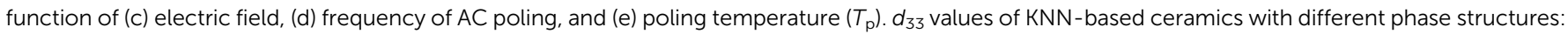

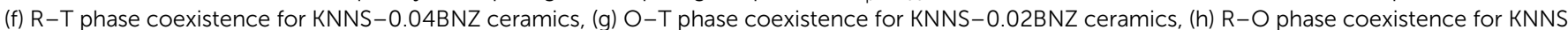

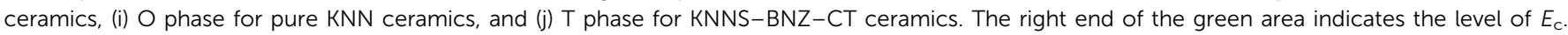

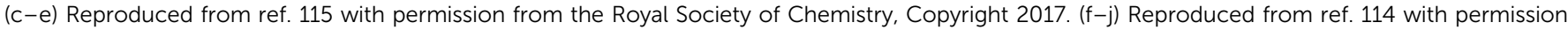
from the American Chemical Society, Copyright 2016.

which was widely reported in BT-based ceramics but rarely studied in KNN-based ceramics because of the difficulty in controlling their grain sizes. ${ }^{180-182}$ Recently, different sintering methods, including hot-press sintering (HPS), microwave sintering (MS), conventional sintering (CS), and two-step sintering (TSS), were used to prepare $0.5 \mathrm{wt} \% \mathrm{MnO}_{2}$-doped $0.95\left(\mathrm{~K}_{0.5} \mathrm{Na}_{0.5}\right)\left(\mathrm{Nb}_{0.965} \mathrm{Sb}_{0.035}\right)$ $\mathrm{O}_{3}-0.01 \mathrm{CaZrO}_{3}-0.04\left(\mathrm{Bi}_{0.5} \mathrm{~K}_{0.5}\right)\left(\mathrm{Hf}_{0.98} \mathrm{Ti}_{0.02}\right) \mathrm{O}_{3}$ (95KNNS-1CZ-4BKHT) lead-free ceramics with an R-T phase boundary, obtaining 95KNNS$1 \mathrm{CZ}-4 \mathrm{BKHT}$ with grain size varying from $0.31 \mu \mathrm{m}$ to $3.31 \mu \mathrm{m} .{ }^{183}$ The results show that $d_{33}$ rapidly increased from $\sim 50 \mathrm{pC} \mathrm{N}^{-1}$ to $\sim 300 \mathrm{pC} \mathrm{N}^{-1}$ as the grain size increased from $0.31 \mu \mathrm{m}$ to $1.04 \mu \mathrm{m}$, then increased up to $\sim 450 \mathrm{pC} \mathrm{N}^{-1}$ as the grain size increased up to $3.04 \mathrm{pC} \mathrm{N}^{-1}$, and finally almost remained unchanged as the grain size further increased to $3.31 \mu \mathrm{m}$ (see Fig. 17(a)). The analysis manifested a similar tendency between $d_{33}$ and $\varepsilon_{\mathrm{r}} P_{\mathrm{r}}$ (see Fig. 17(a)). ${ }^{183}$ Then, the Rayleigh law indicated a higher extrinsic contribution in the samples with larger grain size (see Fig. 17(b)), suggesting more domain wall motion and domain switching. It was inferred that the observed piezoelectricity enhancement was mainly ascribed to the improved dielectric permittivity, ferroelectricity as well as the improved extrinsic contribution. Therefore, controlling the grain size is also another important way to promote the piezoelectric properties of KNN-based ceramics with NPB.

3.1.3 Physical mechanisms. When explaining the physical mechanisms of piezoelectric properties, both intrinsic and extrinsic contributions are considered. ${ }^{71,184-187}$ Herein, we will emphasize the physical mechanisms of piezoelectricity enhancement with regard to these two factors. Generally, the piezoelectric coefficient of perovskite ferroelectrics can be calculated from the equation $d_{33}=2 Q \varepsilon_{33} P_{\mathrm{s}}$, where $Q$ is the electrostrictive coefficient, $\varepsilon_{33}$ is the dielectric permittivity, and $P_{\mathrm{s}}$ is the spontaneous polarization. ${ }^{81,89,91} Q$ was reported to be closely related to the degree of order in cation arrangement. $Q$ will reduce with the increasing degree of disorder in cation arrangement. ${ }^{188,189}$ As discussed above, it is necessary to dope some indispensable
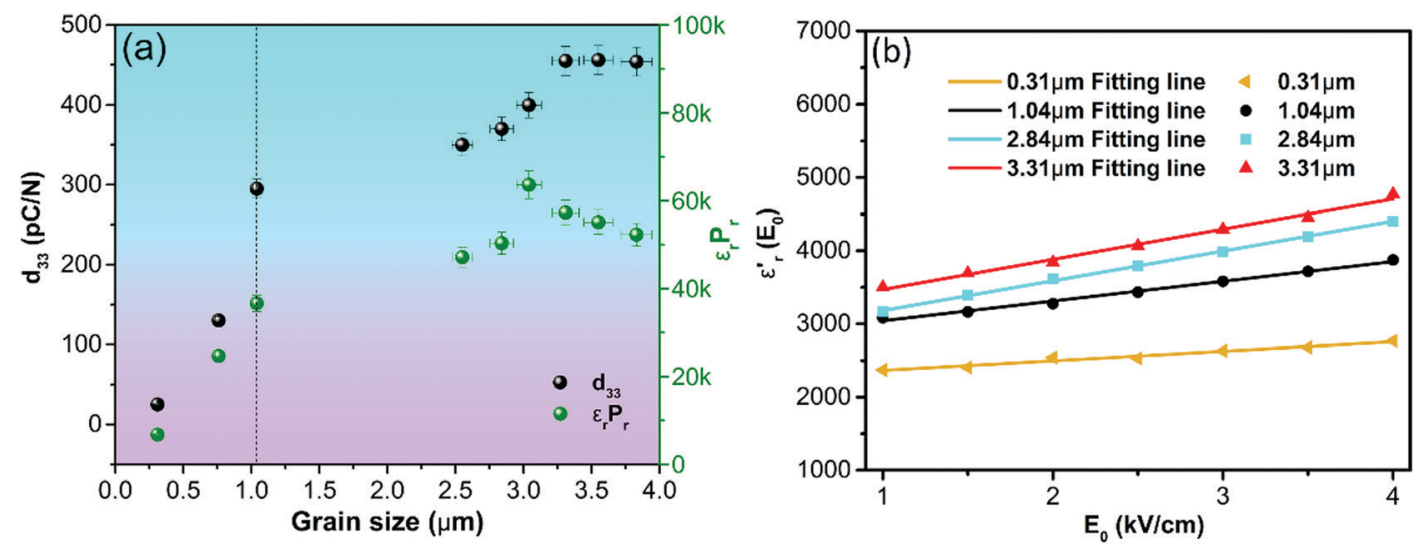

Fig. 17 (a) $d_{33}$ Vs. $\varepsilon_{\mathrm{r}} P_{\mathrm{r}}$ and (b) $\varepsilon_{\mathrm{r}} v s$. $E_{0}$ of $95 \mathrm{KNNS}-1 \mathrm{CZ}-4 \mathrm{BKHT}$ ceramics with different grain sizes. Reproduced from ref. 183 with permission from WileyVCH, Copyright 2019. 
additives into KNN ceramics for constructing the NPB. ${ }^{10}$ But the addition of these additives will inevitably destroy the degree of order of cations, resulting in an increase in the degree of disorder of cations and then a decrease in the $Q$ value. ${ }^{188,189}$ Particularly, the reported publications so far show that the substitution of B-site $\mathrm{Nb}^{5+}$ is compulsory for constructing a NPB, such as by using $\mathrm{Zr}^{4+}, \mathrm{Hf}^{4+}, \mathrm{Sb}^{5+}, \mathrm{Ta}^{5+}$, and so on. ${ }^{10}$ Meanwhile, the destruction of the long-range order caused by the additives will also deteriorate the ferroelectric properties, resulting in reduced $P_{\mathrm{s}} \cdot{ }^{188,189}$ That is to say, the increased $d_{33}$ values mainly originated from the increase of $\varepsilon_{33}$. Indeed, $\varepsilon_{\mathrm{r}}$ of KNN-based ceramics with NPB was even 6.7 times as large as that of the ones with no phase boundaries at room temperature (see Fig. 18(a)). The increase of $\varepsilon_{33}$ was due to shifting of the phase transition temperatures (e.g., $T_{\mathrm{R}-\mathrm{O}}$ and $\left.T_{\mathrm{O}-\mathrm{T}}\right)$ to room temperature and the flattened energy landscape connecting two or more ferroelectric phases at the NPB (see Fig. 18(a)-(c)). ${ }^{32,81,91,111,170}$ Meanwhile, the phase-field simulation further proved a low energy barrier $\left(\sim 10 \mathrm{~J} \mathrm{~cm}^{-3}\right)$ among the two or three phases at the multi-phase coexistence zone (see Fig. 18(b)), indicating a greatly decreased polarization anisotropy (see Fig. 18(c)). ${ }^{24,32}$ Therefore, the construction of the NPB was the main origin of the enhanced piezoelectric properties. On the one hand, the multi-phase coexistence at the NPB possesses more possible polarization variants that provide more possible polarization rotation routes; ${ }^{100,190}$ on the other hand, the flattened energy landscape of multi-phase coexistence guarantees an easy polarization rotation and a high $\varepsilon_{33}$, consequently leading to a high $d_{33}{ }^{24,32,81,91,111,170}$

The low energy barrier mentioned above provides the possibility of achieving high piezoelectric properties. The macro piezoelectricity can be induced by applying an external electric field on KNN-based ceramics. Therefore, the analysis of the phase structure before and after poling is crucial for understanding the physical mechanisms. Fig. 19(a) shows the $\varepsilon^{\prime}-T$ and $\varepsilon^{\prime \prime}-T$ curves of KNNS-SZ- $x$ BAZ $(x=0.035)$ ceramics that exhibited a typical NPB. Before poling, $T_{\mathrm{R}-\mathrm{O}}$ and $T_{\mathrm{O}-\mathrm{T}}$ converged near room temperature and formed a diffused $T_{\mathrm{R}-\mathrm{O} \& \mathrm{O}-\mathrm{T}}$, which was a typical phenomenon when constructing a NPB. ${ }^{24-27,32}$ After poling, $\varepsilon^{\prime}-T$ curves still exhibited the converged $T_{\mathrm{R}-\mathrm{O} \& \mathrm{O}-\mathrm{T}}$, while $\varepsilon^{\prime \prime}-T$ curves displayed the separated $T_{\mathrm{R}-\mathrm{O} \& \mathrm{O}-\mathrm{T}} \cdot T_{\mathrm{O}-\mathrm{T}}$ was about $5{ }^{\circ} \mathrm{C}$, while $T_{\mathrm{R}-\mathrm{O}}$ was too diffused to obtain the exact temperature point. Therefore, it was inferred that the $\mathrm{R}$ phase did exist in both unpoled and poled samples in the form of a diffused phase (i.e., R phase-related PNRs), while the long-range ordered ferroelectric domains possessed an $\mathrm{O}-\mathrm{T}$ coexistence phase. The status of the $\mathrm{R}$ phase was re-arranged and strengthened by an external electric field, resulting in the separation of $T_{\mathrm{R}-\mathrm{O}}$ and $T_{\mathrm{O}-\mathrm{T}}$. A similar tendency was also reported in 50BCT-50BZT ceramics. ${ }^{191}$ Brajesh et al. reported that both unpoled and poled 50BCT-50BZT ceramics possessed the diffused $\mathrm{R}-\mathrm{O}-\mathrm{T}$ phase coexistence, and the content of the $\mathrm{R}$ phase was increased after poling. ${ }^{191}$ The corresponding phase-field simulations also supported the inference. Before poling, the R, $\mathrm{O}$, and $\mathrm{T}$ phases exhibited a similar energy barrier like the situation of KNNS-BNKZ- $x$ AS-Fe $(x=1.6 \%)$ ceramics (see Fig. 18(b)), and the $\mathrm{O}$ phase possessed the lowest energy due to $T_{\mathrm{R}-\mathrm{O} \& \mathrm{O}-\mathrm{T}}$ close to room temperature and the high content of the O phase (see Fig. 19(b)). After poling, the energy gap among $\mathrm{R}, \mathrm{O}$, and $\mathrm{T}$ phases was significantly increased (see Fig. 19(c)). The $\mathrm{O}$ phase still possessed the lowest energy while the $\mathrm{T}$ phase exhibited the highest energy, which was attributed to $T_{\mathrm{O}-\mathrm{T}}$ $\left(5{ }^{\circ} \mathrm{C}\right)$ slightly lower than room temperature. On the other hand, the $\mathrm{R}$ phase showed in-between energy, further proving the existence of the $\mathrm{R}$ phase after poling. Therefore, the phasefield simulations were highly consistent with the experiments. In addition, the poled samples exhibited enhanced $\varepsilon^{\prime}$ (about $350)$ with respect to the unpoled ones. The increased $\varepsilon^{\prime}$ was ascribed to the re-oriented domains that were abruptly disordered when heating to $T_{\mathrm{R}-\mathrm{O} \& \mathrm{O}-\mathrm{T}}$, resulting in the enhanced dielectric response. $^{32}$ Therefore, the effect of an electric field on the phase structure is also important for the enhanced piezoelectric properties at the NPB, which is rarely reported yet.

As discussed above, the mesoscopic structure of KNN-based ceramics with NPB featured micron striped domains and nanoscale domains. ${ }^{23,25,26,29,32,104}$ These miniaturized domains were prone to responding to external stimulations (e.g., electric field and stress). ${ }^{23,25,26,29,32,93-96,104,110}$ The detailed domain configurations of KNN-based ceramics with different phase boundaries are discussed in Fig. 10. Herein, we collected the relationship of domain size and $d_{33}$ values, ${ }^{23}$ as listed in Fig. 20(a). It is distinctly found that the smaller the nanodomains, the higher
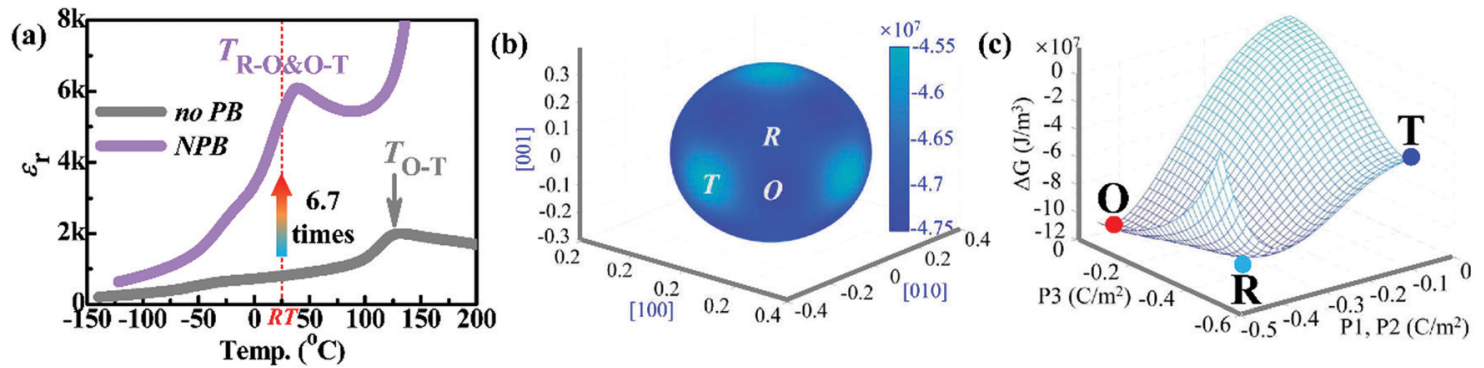

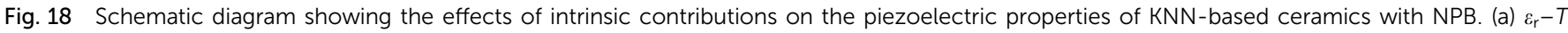

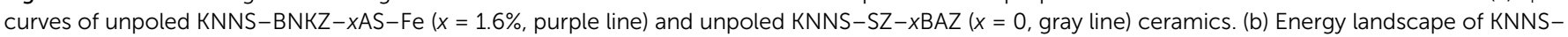

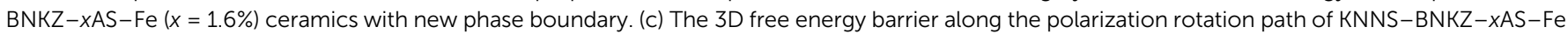

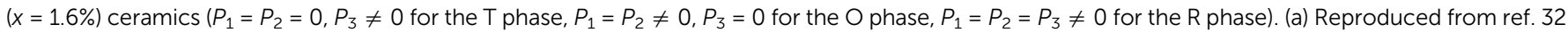

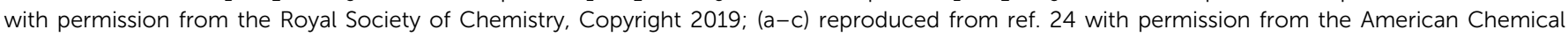
Society, Copyright 2019. 

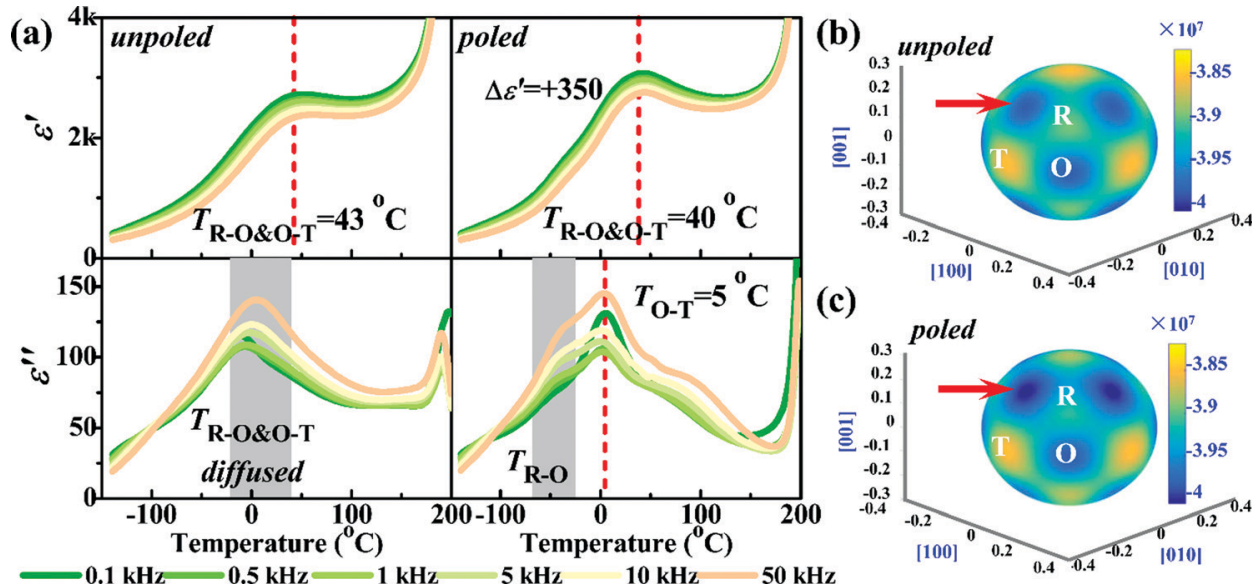

(c)

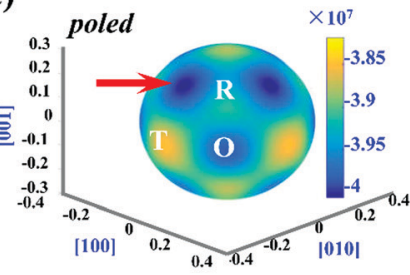

Fig. 19 (a) Temperature-dependent real part $\left(\varepsilon^{\prime}\right)$ and imaginary part $\left(\varepsilon^{\prime \prime}\right)$ of the dielectric permittivity of unpoled and poled KNNS-SZ-xBAZ $(x=0.035)$ ceramics with NPB. Energy landscape of (b) unpoled and (c) poled KNNS-SZ $-x B A Z(x=0.035)$ ceramics. (a-c) Reproduced from ref. 32 with permission from the Royal Society of Chemistry, Copyright 2019.
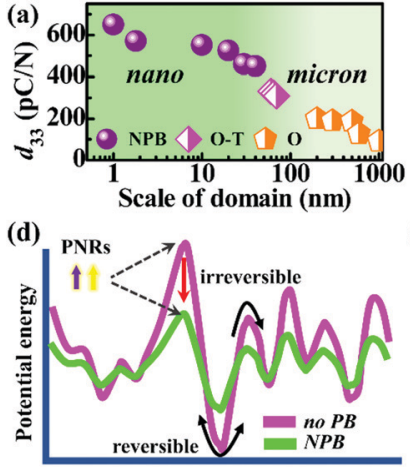

Domain wall position
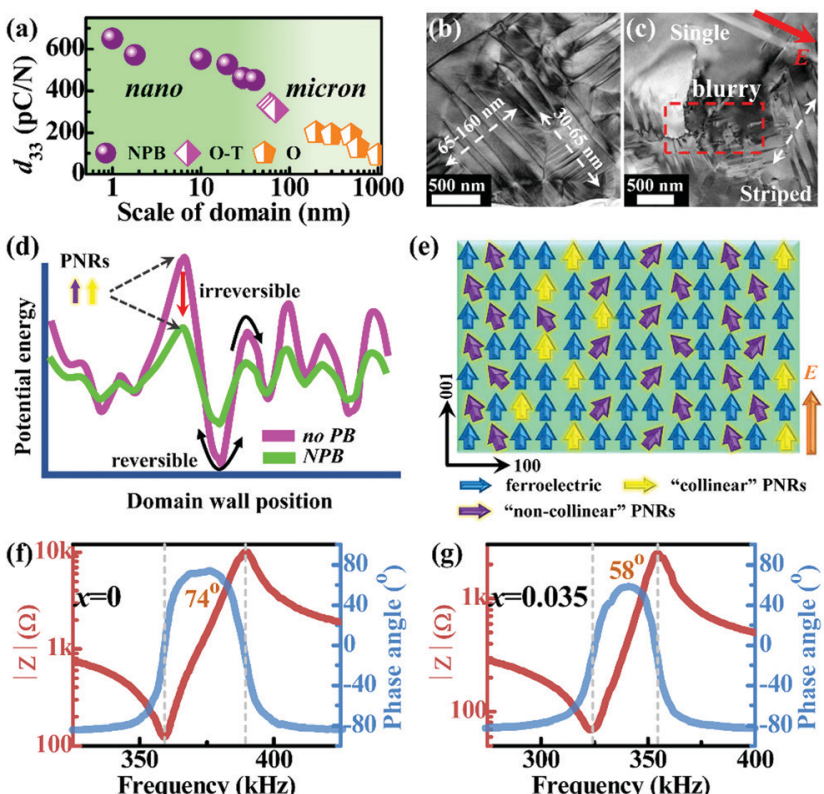

(e)
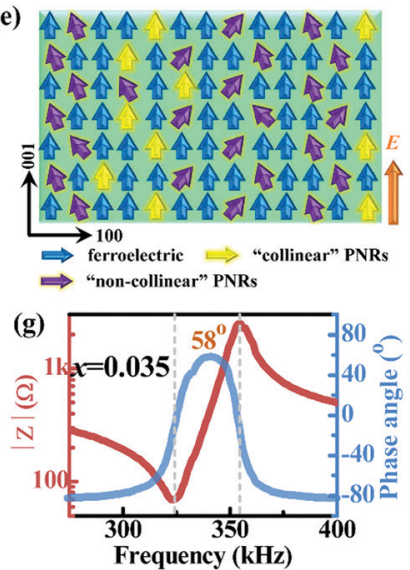

Fig. 20 Schematic diagram showing the effects of intrinsic contributions on the piezoelectric properties of KNN-based ceramics with NPB. (a) Statistics of the relationship between domain size and $d_{33}$ values in KNN-based ceramics. Domain feature of (b) unpoled and (c) poled KNNS-SZ-xBAZ $(x=0.035)$ ceramics with NPB. (d) The energy barrier for domain switching and domain wall motion of $\mathrm{KNN}-\mathrm{SZ}-x \mathrm{BAZ}$ ceramics without phase boundary $(x=0$, magenta line) and with new phase boundary $(x=0.035$, green line). (e) The simulated microstructure of KNN-based ceramics with new phase boundary after poling along the $\langle 001\rangle_{p c}$ direction. Impedance and phase angle of poled KNNS-SZ-xBAZ ceramics with ( $f$ ) $x=0$ and (g) $x=0.035$. (a) Reproduced from ref. 23 with permission from Wiley-VCH, Copyright 2016; (b-g) reproduced from ref. 32 with permission from the Royal Society of Chemistry, Copyright 2019

the $d_{33}$ values. Thus, the ceramics with NPB exhibited high $d_{33}$ values (400-570 $\mathrm{pC} \mathrm{N}^{-1}$ ) due to the relatively small size of nanodomains. ${ }^{23,26,28}$ More surprisingly, KNNS-BNKZ- $x$ AS-Fe $(x=1.6 \%)$ ceramics even possessed an ultra-high $d_{33}$ value of $650 \pm 20 \mathrm{pC} \mathrm{N}^{-1}$ due to the ultra-thin nanodomains. ${ }^{24}$ As discussed in Section 2.2, smaller domains originate from lower domain wall energy. ${ }^{87}$ The flattened energy landscape connecting different phases within the NPB allows a low domain wall energy, resulting in the miniaturized domain size. ${ }^{87}$ But however, the previously reported domains of $\mathrm{KNN}$-based ceramics were mainly obtained from the unpoled samples. ${ }^{23,25,26,29,104}$ However, the net piezoelectricity can be observed after applying an external electric field that will inevitably induce domain switching and domain wall motion. ${ }^{192}$ Although chemical etching is utilized to observe the domains of poled KNN-based ceramics, this method could irreversibly destroy the subtle domains (e.g., nano-domains). ${ }^{68,98,104,193}$ Fortunately, the combined use of TEM and focused ion beam (FIB) could precisely obtain the domain structures of both unpoled and poled KNN-based ceramics without significantly destroying the feature. ${ }^{32}$ The unpoled KNNS-SZ- $x$ BAZ ceramics $(x=0.035)$ exhibited the typically striped domains with a scale of 30-160 nm (see Fig. 20(b)), ${ }^{32}$ which were widely observed in other KNN-based ceramics with NPB. After poling, three types of domains were observed, that is, striped domains, blurry domains, and single domains (see Fig. 20(c)). ${ }^{32}$ Not surprisingly, the striped domains were re-arranged along the direction of the poling electric field (see Fig. 20(c)). ${ }^{32}$ It should be pointed out that such a re-arrangement was not absolute due to the interaction among domains and grains, which was different from the situation of domain engineered piezoelectric single crystals. ${ }^{3,17}$ Of particular interest is that the single domain zone was observed in poled samples (see Fig. 20(c)). ${ }^{32}$ Such a unique single domain structure was also observed in high-performance 0.50BZT-0.50BCT ceramics by means of in situ TEM and was believed to be responsible for the piezoelectricity enhancement. ${ }^{192}$ It was mentioned that such a single domain structure was only observed in a very particular electric field. ${ }^{192}$ On the other hand, the complicated domain structures in bulk ceramics may provide such an environment under an electric field, resulting in the occurrence of a single domain structure. More importantly, the blurry domains were observed at the junction of striped domains and single domain zone, which was 
believed to be related to the local structural heterogeneity (e.g., PNRs) caused by the addition of additives (see Fig. 20(c)). ${ }^{32}$ Recently, TEM was used to successfully visualize the PNRs that exhibited the scale of 5-20 nm. ${ }^{29,192}$ PNRs acted as a "seed" or an "accelerator" during the domain switching and lowered the energy barrier for domain switching, promoting the process of domain switching and domain wall motion. ${ }^{29}$ Here, we'd like to emphasize that the occurrence of PNRs was not completely good for the piezoelectric properties of KNN-based ceramics. Although PNRs could promote the domain switching, it is very difficult to achieve a saturated poling due to the large energy difference of PNRs and the ferroelectric matrix and the high content of "non-collinear" PNRs. ${ }^{32,81,192}$ That is the reason why the phase angle of KNN-based ceramics with NPB was reported to be lower than that of the counterparts without NPB (see Fig. 20(f) and (g)). ${ }^{32}$ In other words, a high $d_{33}$ value is highly expected if one can achieve a highly saturated poling at the NPB, which could be obtained by elaborately tailoring the composition design. Therefore, it can be concluded that the piezoelectricity enhancement at the NPB mainly originated from the enhanced dielectric response, the easy polarization rotation and the facilitated domain switching.

Based on the aforementioned results and discussions, we summarized the physical mechanisms of the enhanced piezoelectric properties of KNN-based ceramics with NPB, as listed in Fig. 21. The intrinsic contribution from multi-phase coexistence plays a dominant role in the piezoelectric properties due to the large dielectric response and the low polarization anisotropy. The intermediate phase induced by the external stimulus (e.g., electric field) could further promote the polarization rotation. Furthermore, the miniaturized domains are prone to switching under an electric field, benefiting the net piezoelectric properties. The role of local heterogeneity (e.g., PNRs) is two-edged. An appropriate amount of PNRs will promote the domain switching, while a higher content of PNRs will hinder the full poling due to the large energy difference of the ferroelectric matrix and PNRs. Appropriately increasing the grain size will enhance the permittivity and promote the domain wall motion, which also benefits the macro piezoelectric properties. Actually, Fig. 21 can also guide the future work on acquiring higher piezoelectric properties in $\mathrm{KNN}$ based ceramics. First of all, the construction of the NPB is indispensable due to the dominant role of multi-phase coexistence. Then, other methods (including composition design, fabrication technology, and sintering aids) are needed to satisfy the other factors as many as possible.

\subsection{Strain properties}

3.2.1 Current strain properties at the NPB. The strain is the displacement or distortion of a piezoceramic with respect to an external stimulus (e.g., electric field or stress), which is applied in displacement control (e.g., piezoelectric actuators). ${ }^{12,38,188,194}$ The previous reviews have systematically summarized the strain properties of lead-free piezoceramics (e.g., BNT-, BT-, and KNNbased ceramics). ${ }^{38}$ For KNN-based ceramics, they mainly discussed the effects of chemical modification, texturing, and domain engineering on the strain properties of KNN-based ceramics, without systematically summarizing the effects of the NPB on the strain properties of KNN-based ceramics and its related physical mechanisms. ${ }^{38}$ Particularly, the previous reviews did not analyze the strain properties of KNN-based ceramics from the fundamental basis, such as the measurement process. ${ }^{38,194}$ Such neglect would greatly hinder the understanding of strain properties of KNN-based ceramics. Herein, we mainly focus on the effects of the NPB on the strain properties of KNN-based ceramics and the related physical mechanisms. Fig. 22(a)-(f) show the typical unipolar and bipolar strain curves of poled KNNS-SZ- $x$ BNZ ceramics, in which the different phase boundaries (e.g., $x=0$ for O phase, $x=0.02$ for O-T phase boundary, and $x=$ 0.03 for NPB) could be obtained by varying the $x$ value. $^{30,66}$ As the phase structure changed from a single $O$ phase to a multi-phase coexistence (i.e., O-T and $\mathrm{R}-\mathrm{O}-\mathrm{T}$ coexistence phase), the unipolar strain $\left(S_{\text {uni }}\right)$, positive strain $\left(S_{\text {pos }}\right)$ and negative strain $\left(S_{\text {neg }}\right)$ increased. Particularly, the poled KNNS-SZ- $x$ BNZ $(x=0.03)$ ceramics possessed the largest strain response. Since the strain level is highly related to the amplitude of the electric field, the researchers used the equation $d_{33^{*}}=S_{\max } / E_{\max }$ (where $S_{\max }$ is the maximum

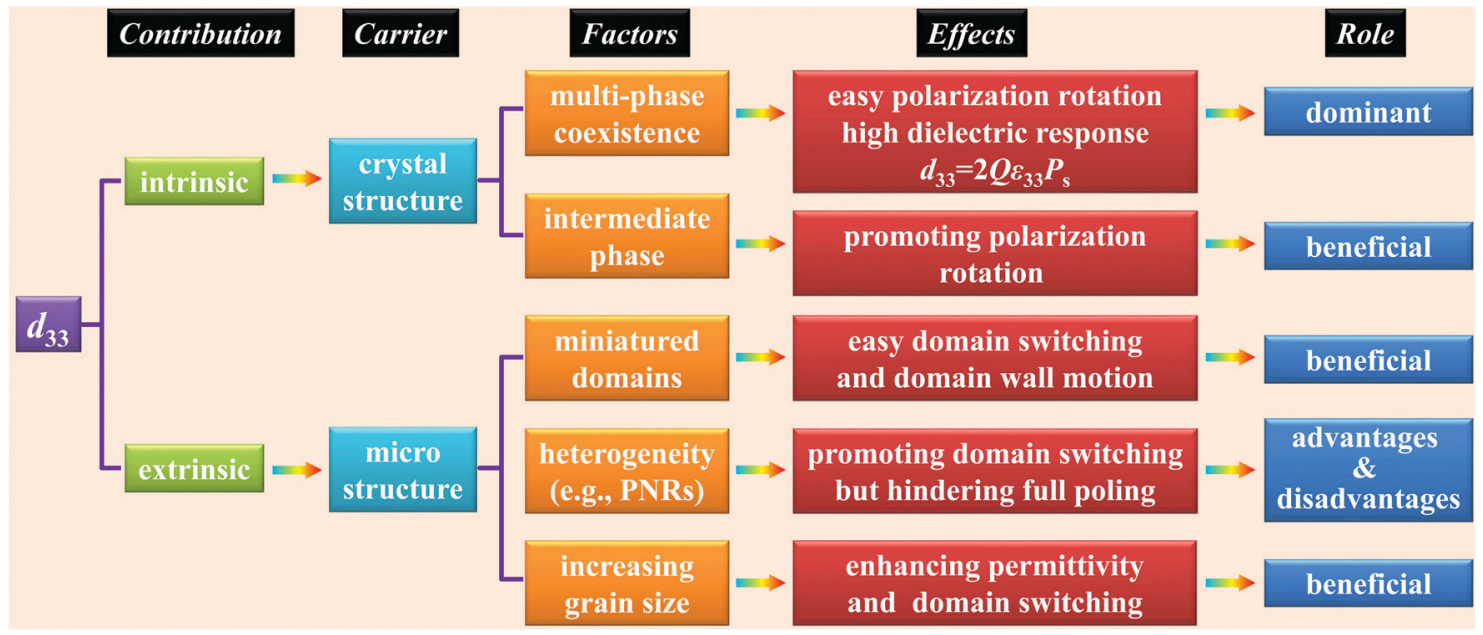

Fig. 21 A schematic diagram describing the physical mechanisms of the enhanced piezoelectric properties of KNN-based ceramics with NPB. 

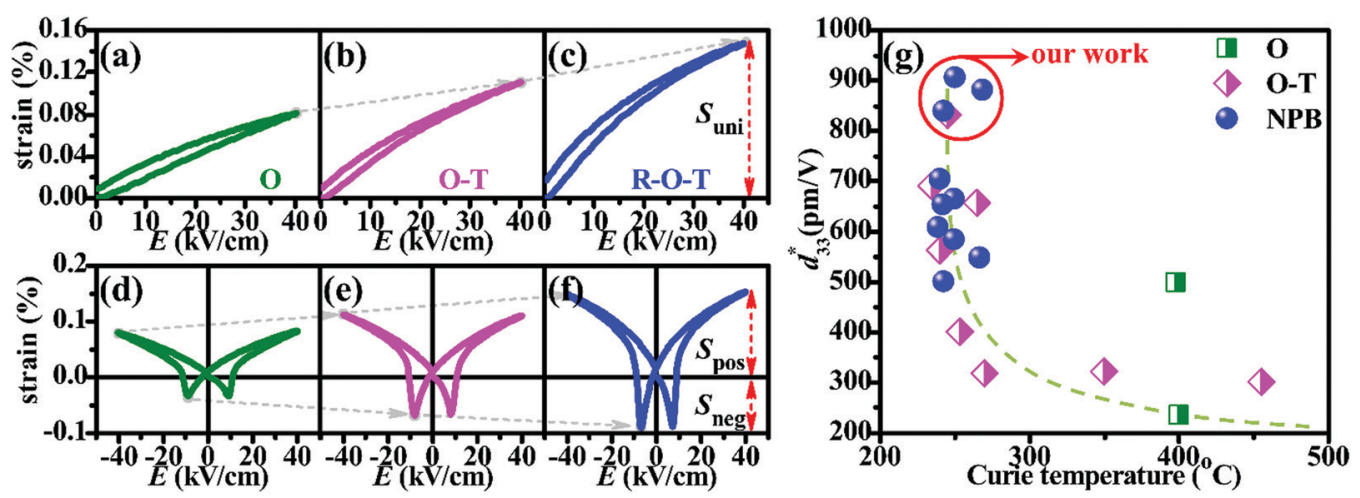

Fig. $22(\mathrm{a}-\mathrm{c})$ Unipolar and $(\mathrm{d}-\mathrm{f})$ bipolar strain curves of KNNS-SZ-xBNZ ceramics with $x=0$ ( $\mathrm{a}$ and d), $x=0.02$ (b and e), and $x=0.03$ (c and f). (g) $d_{33}{ }^{*}$ vs. $T_{c}$ of KNN-based ceramics with different phase boundaries. (a-c) Reproduced from ref. 30 with permission from Wiley-VCH, Copyright 2019; (d-f) reproduced from ref. 66 with permission from the Royal Society of Chemistry, Copyright 2018; (g) reproduced from ref. 38 with permission from Elsevier, Copyright 2019

strain at the maximum electric field $\left(E_{\max }\right)$ and $d_{33^{*}}$ is called the converse piezoelectric coefficient) to evaluate the strain response of ferroelectrics. ${ }^{91,193}$ Theoretically, $d_{33}{ }^{*}$ should be equal to $d_{33}$ at a low electric field. ${ }^{80,111,170}$ However, the reported $d_{33}{ }^{*}$ values were generally higher than $d_{33}$ due to the domain switching and domain wall motion at a high electric field. ${ }^{80,111,170}$ Thus, $d_{33^{*}}$ is actually the normalized strain to the electric field. Hao et al. collected the $d_{33^{*}} v s$. $T_{\mathrm{c}}$ distribution in KNN-based ceramics with different phase boundaries, ${ }^{38}$ as shown in Fig. $22(\mathrm{~g})$. Apparently, KNN-based ceramics with multi-phase coexistence occupied the upper left area while the ones with a single $O$ phase occupied the lower right area. Furthermore, KNN-based ceramics with NPB exhibited higher $d_{33}{ }^{*}$ values than those of the ones with $\mathrm{O}-\mathrm{T}$ coexistence phase, while showing the same or similar $T_{\mathrm{c}}$ values. In addition, our group obtained enhanced $d_{33}{ }^{*}$ values in KNN-based ceramics by constructing the NPB and by optimizing the composition design (see Fig. $22(\mathrm{~g})$ ). Therefore, the NPB could further promote the strain properties of KNNbased ceramics when compared with the $\mathrm{O}-\mathrm{T}$ phase boundary.

Here, the unipolar strain curves of representative lead-based and lead-free piezoceramics were collected, ${ }^{20,30,39,66,195-197}$ as shown in Fig. 23. BNT-based ceramics were reported to exhibit high strain arising from the electric field-induced ferroelectricrelaxor phase transition; however the high strain hysteresis, the large electric field necessary to induce the high strain and the strong temperature dependence of strain strictly restricted their practical applications. ${ }^{20} \mathrm{KNN}$-based ceramics with NPB exhibited a comparable strain to those of BT- and BF-based ceramics, accompanied with relatively moderate strain and low strain hysteresis. Considering the relatively high $T_{\mathrm{c}}\left(\geq 200{ }^{\circ} \mathrm{C}\right)$, KNNbased ceramics with NPB have promising potential for piezoelectric actuator applications. However, it should also be pointed out that there is still an obvious gap between KNN-based ceramics and lead-based counterparts with respect to strain. Thus, further investigations on the strain of KNN-based ceramics are still urgently needed.

3.2.2 Physical mechanisms. Generally, the strain generated in ferroelectrics under an electric field originates from (i) the converse piezoelectric effect, (ii) non- $180^{\circ}$ domain switching

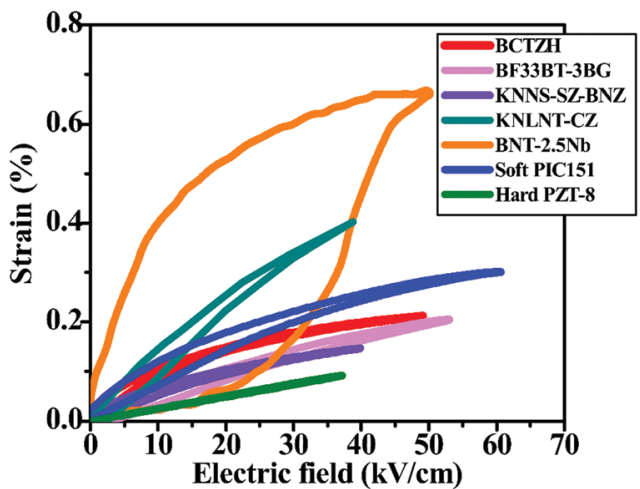

Fig. 23 Comparison of strain properties of both lead-based and lead-free piezoceramics. Unipolar strain curves of representative lead-free and leadbased piezoceramics. Reproduced from ref. 20 with permission from Wiley-VCH, Copyright 2016; reproduced from ref. 30 with permission from the Royal Society of Chemistry, Copyright 2019; reproduced from ref. 39 with permission from Wiley-VCH, Copyright 2015; reproduced from ref. 66 with permission from Wiley-VCH, Copyright 2018; reproduced from ref. 195 with permission from the Springer Nature Publishing Group, Copyright 2012; reproduced from ref. 196 with permission from the American Chemical Society, Copyright 2012; reproduced from ref. 197 with permission from Elsevier, Copyright 2018.

and domain wall motion, (iii) electrostriction, and (iv) possible electric field-induced phase transition. ${ }^{12,30,66,143,198,199}$ Before discussing the physical mechanisms behind NPB affecting the strain properties of KNN-based ceramics, we'd like to briefly describe the general process for strain measurement. The pure electrostriction materials are widely used for the precise displacement control due to the absence of strain hysteresis, no need for poling and the independence of other conditions (such as grain size, temperature, operating frequency and so on). ${ }^{188,189,200,201}$ But however, the ferroelectric ceramics need a full poling before being used for the precise displacement control due to the existence of non- $180^{\circ}$ ferroelastic domains that will cause a distinct strain hysteresis during the domain switching and domain wall motion. ${ }^{9,12,195}$ Considering the limited breakdown field strength, it is better to not directly apply a high electric field on the ferroelectric ceramics. Thus, the most common 
step for measuring unipolar strain is to gradually increase the electric field to protect the samples from breakdown, as shown in Fig. 24(a). Such a step will gradually re-orientate the domains along the direction of the electric field (see the inset of Fig. 24(a)). The finally measured unipolar strain comes from the samples that were poled at a high electric field, suggesting the absent contribution from irreversible non $-180^{\circ}$ domain switching. This conclusion was well proved in commercial soft PIC151 ceramics and KNN-based ceramics. ${ }^{198}$ As shown in Fig. 24(b), the unpoled PIC151 ceramics exhibited an apparent and large remanent strain $\left(S_{\text {rem }}\right)$ that was induced by the irreversible non- $180^{\circ}$
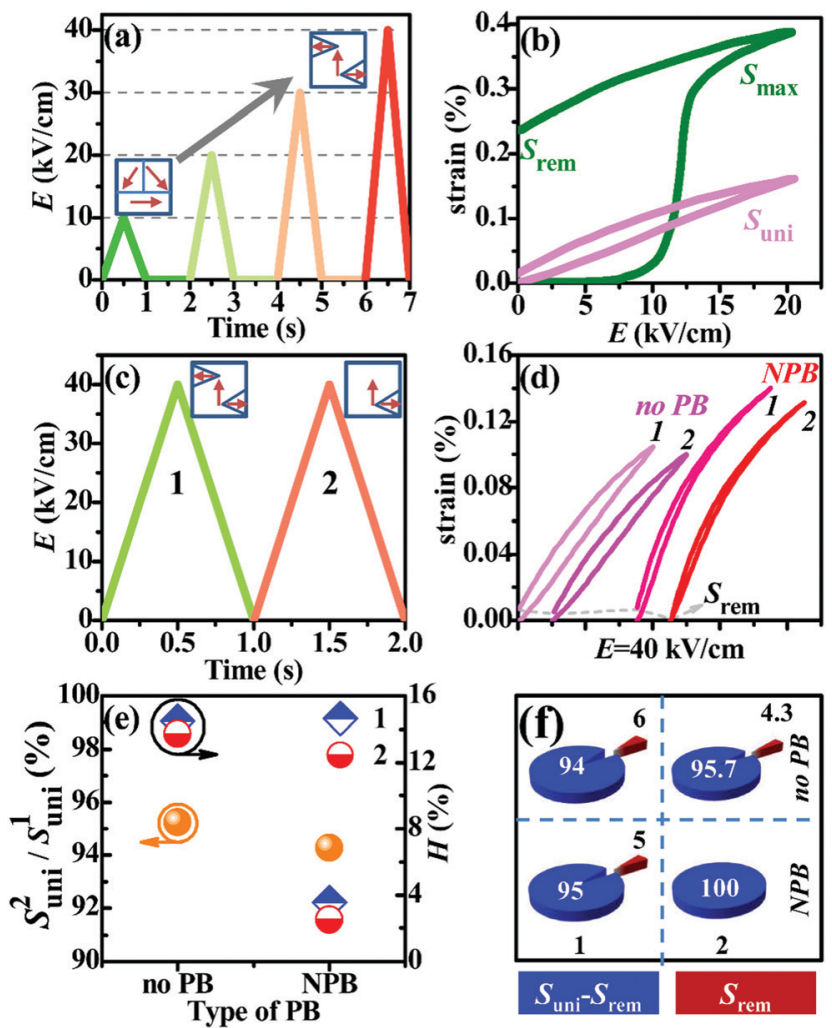

Fig. 24 The physical model describing the origin of enhanced strain properties in KNN-based ceramics with NPB. (a) Usual step of the applied electric field for measuring unipolar strain. (b) Unipolar strain curves of the first (green line) and second (pink line) electric field of commercial PIC151 ceramics. (c) Electric field for measuring the unipolar strain curves of poled KNN-based ceramics without phase boundary and with NPB. " 1 " and " 2 " mean the first and second electric cycle. (d) Unipolar strain curves of poled KNN-based ceramics without phase boundary and with NPB under the electric field in (c). (e) $S_{\text {uni }}^{2} / S_{\text {uni }}^{1}$ and strain hysteresis ( $H=\frac{\Delta S_{E_{\max } / 2}}{S_{\max }} \times 100 \%$, $S_{\max }$ is the unipolar strain and $\Delta S_{E_{\max } / 2}$ is the strain difference at half of the maximum electric field). (f) Percentage of $S_{\text {uni }}-S_{\text {rem }}$ and $S_{\text {rem }}\left(S_{\text {uni }}\right.$ is the unipolar strain and $S_{\text {rem }}$ is the remanent strain) measured on the first and second electric field of poled KNN-based ceramics without phase boundary and with NPB. The insets of ( $a$ and $c$ ) show the simulations of ferroelectric domains. When increasing the electric field from low to high level, the domains will gradually re-orientate along the direction of the electric field. For the inset of (c), a further increase of the electric field can result in a higher degree of re-orientation of domains. (b) Reproduced from ref. 198 with permission from Elsevier, Copyright 2015; (d-f) reproduced from ref. 143 with permission from the Royal Society of Chemistry, Copyright 2018. domain switching, while the poled ones showed a negligible $S_{\text {rem }}{ }^{198}$ More importantly, the difference value between $S_{\max }$ and $S_{\text {rem }}$ (of the unpoled PIC151 ceramics) is identical to $S_{\text {uni }}$ (of the poled PIC151 ceramics). ${ }^{198}$ Our group further proved the origin of $S_{\text {uni }}$ (as widely reported in the literature) by applying a successive and high electric field to poled KNN-based ceramics without phase boundary and with NPB, as simulated in Fig. 24(c). ${ }^{30,143}$ The results showed that the poled KNN-based ceramics without phase boundary exhibited a visible $S_{\text {rem }}$ on both electric cycles, while the ones with NPB showed a $S_{\text {rem }}$ only on the first electric cycle and no $S_{\text {rem }}$ on the second one (see Fig. 24(d)). ${ }^{30,143}$ Furthermore, the calculated $S_{\text {uni }}^{2} / S_{\text {uni }}^{1}$ values showed that both samples exhibited a high retention of $\geq 94 \%$, indicating the full poling before measurement and the negligible irreversible $180^{\circ}$ domain switching (see Fig. 24(e)). ${ }^{30,143}$ More importantly, the poled KNN-based ceramics with NPB exhibited a much lower $H$ value $(\leq 4 \%)$ than that of the ones without phase boundary, suggesting the negligible non- $180^{\circ}$ domain switching (see Fig. 24(e) ). ${ }^{30,143}$ The relatively high $H$ value in the poled KNNbased ceramics without phase boundary was likely related to the reversible $180^{\circ}$ domain switching. ${ }^{12,30,66,143,198,199,202}$ Finally, the percentage of $\left(S_{\text {uni }}-S_{\text {rem }}\right)$ and $S_{\text {rem }}$ is listed in Fig. 24(f). The poled KNN-based ceramics with NPB exhibited a very low $S_{\text {rem }}$ (even no $S_{\text {rem }}$ on the second electric cycle) and $H$, indicating the potential in precise displacement control. Therefore, the reported $S_{\text {uni }}$ of KNN-based ceramics mainly originated from the intrinsic contribution (e.g., the converse piezoelectric response). ${ }^{30,143}$

To clearly show how the NPB affects the strain properties, we depicted a mode, as shown in Fig. 25. Obviously, the four aforementioned factors can benefit strain properties. In principle, both the converse piezoelectric response and the non- $180^{\circ}$ domain switching play an important role in the strain of unpoled $\mathrm{KNN}$ based ceramics. ${ }^{12,30,66,143,198,199}$ But however, the most reported $S_{\text {uni }}$ of KNN-based ceramics was obtained from the fully poled samples, in which the irreversible non- $180^{\circ}$ domain switching is completed when applying a gradually increasing electric field. Therefore, the observed enhancement of $S_{\text {uni }}$ in KNN-based ceramics with NPB mainly originated from the converse piezoelectric response due to the multi-phase coexistence that was prone to responding to the electric field. ${ }^{12,30,66,143,198,199}$ In addition, the reversible $180^{\circ}$ domain switching and the electrostriction effect also played a partial role in the enhanced strain properties of KNN-based ceramics with NPB. The question that is left is whether the electric field-induced phase transition (EIPT) occurs or not. PZT based ceramics with MPB were reported to possess an electric field-induced phase transition among R, monoclinic (M), and $\mathrm{T}$ phases due to the low energy landscape among different ferroelectric phases. ${ }^{92,203,204}$ The multi-phase coexistence in KNN-based ceramics with NPB also provided the possible conditions for EIPT. Therefore, it is highly expected to find the evidence of EIPT in KNN-based ceramics with NPB, which has not been reported so far and is one of the future research works. Actually, Fig. 25 not only provides a way to distinguish the factors affecting the strain properties of KNN-based ceramics but also provides the way to obtain better strain properties in both KNN-based ceramics and other 


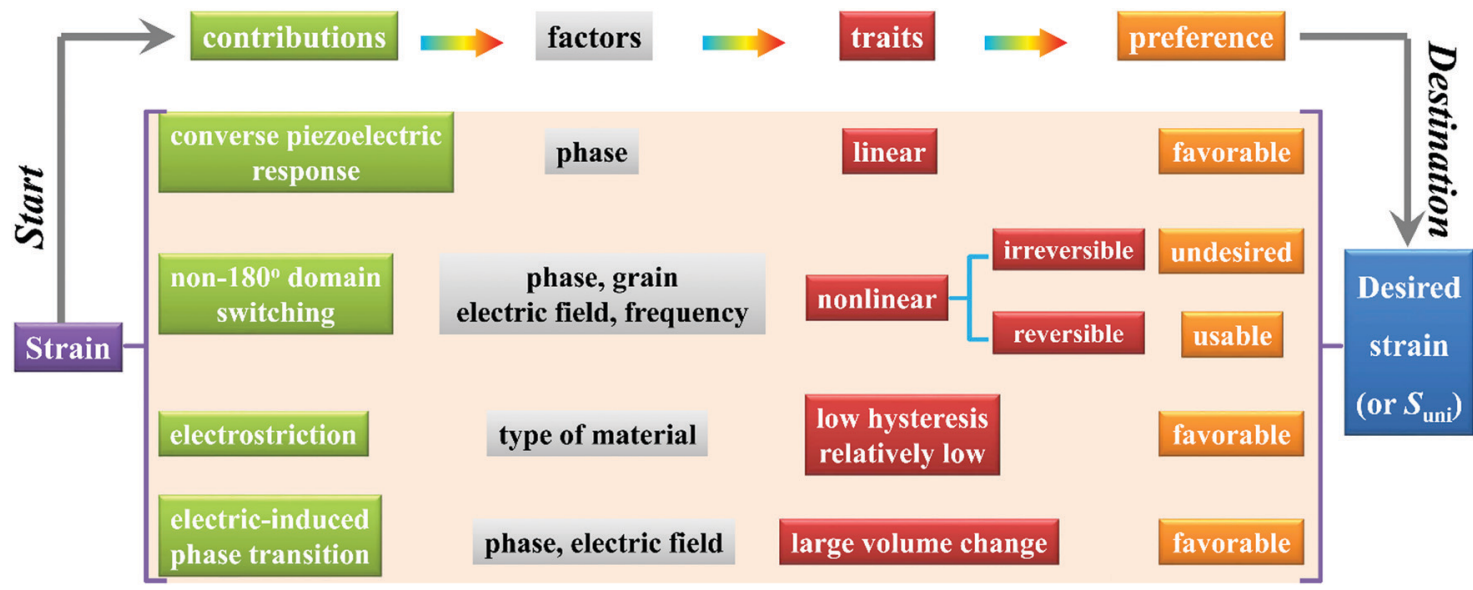

Fig. 25 Way to obtain the desired strain in non-textured KNN-based ceramics. The converse piezoelectric response is dependent on the crystal lattice that is reflected by the phase structure. Non- $180^{\circ}$ domain switching can be classified into two types, that is, irreversible and reversible. The irreversible non- $180^{\circ}$ domain switching contributed to the strain only on the first electric cycle if applying a high electric field, while the reversible one occurred on every electric cycle. The non- $180^{\circ}$ domain switching is strongly dependent on the phase structure, grain size, and measurement conditions (e.g., electric field, frequency, and so on). The electrostriction effect will always exist when applying an electric field. The amplitude of electrostriction is only determined by the piezoceramics themselves and the electric field, and can be calculated from the equation $S=Q P^{2}$, where $Q$ is the electrostriction coefficient and $P$ is the polarization. $Q$ is related to the parent phase of piezoceramics. The strain from electrostriction is relatively much lower than the ones from the converse piezoelectric response and non- $180^{\circ}$ domain switching for a typical ferroelectric material, but shows a hysteresis-free behavior. The reported publications showed that the electric field-induced phase transition also enhanced the strain properties, particularly for the piezoceramics with multi-phase coexistence. ${ }^{92,198,203,204}$ Unfortunately, few publications reported the electric field-induced phase transition in KNN-based ceramics with NPB. Therefore, no clear evidence showed the relationship between electric field-induced phase transition and strain properties in KNN-based ceramics with NPB.

piezoelectric ceramics. For example, Ranjan et al. obtained a giant electrostrain of $1.3 \%$ in $\mathrm{BiFeO}_{3}-\mathrm{PbTiO}_{3}-\mathrm{LaFeO}_{3}$ ceramics by fully considering the contributions from lattice strain, reversible non- $180^{\circ}$ domain switching, and EIPT. ${ }^{205}$ Therefore, the future work on tailoring the strain properties of piezoceramics including both lead-based and lead-free ones can refer to Fig. 25.

\subsection{Temperature stability}

Stability is also another important factor to evaluate piezoelectric materials for practical applications. ${ }^{5,6,9,10,15,38}$ PZT-based ceramics are famous for their exceptional temperature stability, which is the shortcoming of lead-free piezoceramics with PРB. ${ }^{5,6,9,10,15,38}$ Although many publications have reported the enhanced temperature stability of KNN-based ceramics with NPB, a systematic and in-depth understanding is still absent. Herein, we systematically analyze the temperature stability of KNN-based ceramics with NPB in this section, with regard to the measurement methods and physical mechanisms. Up to now, three methods have been used to examine the temperature stability of KNN-based ceramics. We will introduce the methods and discuss their validity, particularly focusing on the temperature stability of KNN-based ceramics at the NPB.

\subsubsection{Measurement methods for temperature stability}

In situ temperature-dependent small signal $d_{33}$. This method is for measuring in situ small signal $d_{33}$ values of poled samples with increasing temperature, as simulated in Fig. 26(a). ${ }^{206}$ Therefore, this method can truly reflect the temperature stability of piezoelectric properties at different surrounding temperature. Using this method, it was found that the commercial soft PIC151 ceramics exhibited a stable $d_{33}$ value at room temperature to near (a)
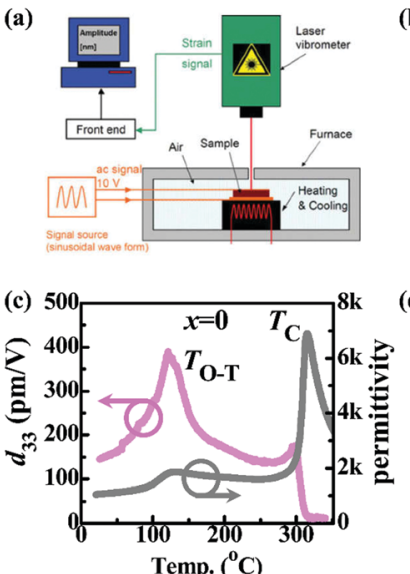

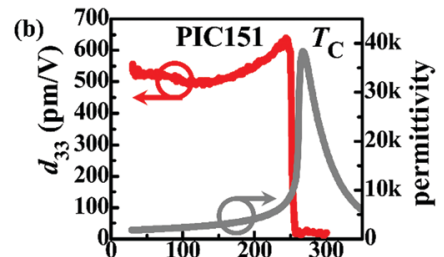

Temp. $\left({ }^{0} \mathrm{C}\right)$

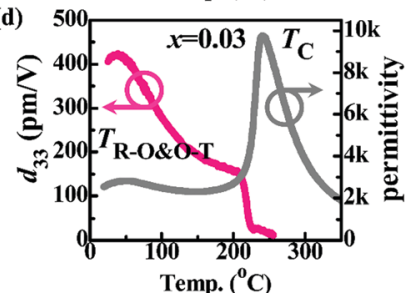

Fig. 26 Temperature stability of PIC151 and KNN-based ceramics. (a) Schematic diagram of the equipment for measuring in situ temperaturedependent small signal $d_{33}$ using a laser. During the heating process, a small ac voltage of $\pm 10 \mathrm{~V}$ was applied on the poled samples, and the computer collected the vibration of the laser and then calculated $d_{33}$ values. The use of such a low ac voltage was to avoid the domain switching and domain wall motion, and reflect the intrinsic piezoelectric properties of piezoceramics. In situ $d_{33}$ values of poled PIC151 ceramics (b), KNNS-SZ-xBNZ ceramics $(x=0)(c)$, and KNNS-SZ-xBNZ ceramics $(x=0.03)$ (d). (a) Reproduced from ref. 206 with permission from Wiley-VCH, Copyright 2012.

$T_{\mathrm{c}}$ as expected due to the trait of MPB (see Fig. 26(b)). In contrast, the poled KNNS-SZ- $x \mathrm{BNZ}$ (e.g., $x=0$ for $\mathrm{O}$ phase and $x=0.03$ for NPB) ceramics displayed an obvious temperature dependence of $d_{33}$ values. The abrupt increase of $d_{33}$ was observed near the ferroelectric-ferroelectric phase transition, while the sharp 
decrease of $d_{33}$ occurred near $T_{\mathrm{c}}$ (see Fig. 26(c) and (d)). Therefore, $d_{33}$ values of the poled KNNS-SZ- $x$ BNZ $(x=0.03)$ ceramics reduced monotonously at $T>50{ }^{\circ} \mathrm{C}$ because of its $T_{\mathrm{O}-\mathrm{T}}$ lower than $50{ }^{\circ} \mathrm{C}$. A similar situation was also reported in other $\mathrm{KNN}$ based ceramics with $\mathrm{O}-\mathrm{T}$ phase boundary and NPB, such as KNNLT-CZ and KNN-BLT-BZ ceramics. ${ }^{70,160}$ The obvious temperature dependence of KNN-based ceramics' $d_{33}$ values mainly originates from the intrinsic trait of the PPB that is closely related to the ambient temperature. ${ }^{61}$ Interestingly, both KNNS-SZ- $x$ BNZ $(x=0$ and $x=0.03$ ) ceramics exhibited a similar $d_{33}$ value of $400-420 \mathrm{pC} \mathrm{N}^{-1}$ at $T_{\mathrm{O}-\mathrm{T}}$, indicating that the enhanced piezoelectric properties of KNN-based ceramics $(x=0.03)$ with NPB mainly originated from its $T_{\mathrm{O}-\mathrm{T}}$ close to room temperature. Therefore, this method further proved that it was NPB that played an important role in the enhancement of KNN-based ceramics' piezoelectric properties.

Here, we collected the in situ small signal $d_{33}$ values of several representative KNN-based ceramics, as shown in Fig. 27. ${ }^{70,160}$ $d_{33}$ values of the samples with $\mathrm{O}-\mathrm{T}$ phase boundary or NPB gradually reduced when the measurement temperature increased from $T_{\mathrm{O}-\mathrm{T}}$ to $T_{\mathrm{c}}$, and rapidly dropped to zero when approaching their $T_{\mathrm{c}}$ values. As mentioned above, it is inevitable for KNN-based ceramics to exhibit the temperature dependence of piezoelectric properties due to the intrinsic trait of the PPB. But however, it should also be pointed out that the NPB not only improved the room-temperature $d_{33}$ values but also guaranteed a relatively high $d_{33}$ value (200$400 \mathrm{pC} \mathrm{N}^{-1}$ ) in the temperature range of room temperature to $150{ }^{\circ} \mathrm{C}$. Moreover, when heating over $150{ }^{\circ} \mathrm{C}, d_{33}\left(\sim 200 \mathrm{pC} \mathrm{N}^{-1}\right)$ values at the NPB can be preserved, but the ones at the O-T phase boundary sharply reduced to zero (see Fig. 27). Therefore, the NPB could provide better temperature stability of in situ small $d_{33}$ values than the $\mathrm{O}-\mathrm{T}$ phase boundary. It should also be pointed out that the exact temperature stability of the NPB was strongly dependent on the composition design, which was the reason why KNNS-SZ- $x \mathrm{BNZ}(x=0.03), 5 \mathrm{BZ} / 3 \mathrm{BLT} / 92 \mathrm{KNN}$ and

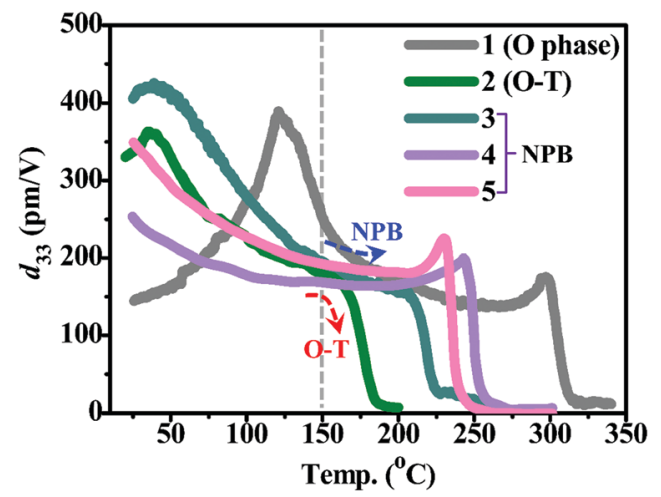

Fig. 27 In situ small signal $d_{33}$ values among different KNN-based ceramics. All data of each composition were measured by using the method described in Fig. 19(a). The gray and cyan lines represent KNNS-SZ-xBNZ ceramics with $x=0$ and $x=0.03$, respectively. The green line represents KNNLT-CZ-Mn (KNN-CZ5) ceramics. The light purple and light pink lines represent 5BZ/3BLT/92KNN and 6BZ/2BLT/92KNN ceramics, respectively. Reproduced from ref. 70 and 160 with permission from Wiley-VCH, Copyright 2013, 2015.
6BZ/2BLT/92KNN ceramics exhibited the different temperature dependencies of in situ small $d_{33}$ values. Currently, the investigations on in situ temperature-dependent $d_{33}$ values of $\mathrm{KNN}$-based ceramics are still relatively few, which should be given more effort.

In situ temperature-dependent strain. The converse piezoelectric effect generates the displacement under an electric field. ${ }^{12,38,188,194}$ Thus, the researchers measured the temperature-dependent strain curves to reflect the temperature stability of piezoceramics. Fig. 28(a) shows the schematic diagram for measuring the temperature-dependent strain curves. It should be mentioned that the samples have been poled actually before heating, as discussed in Fig. 24. During the bipolar strain curve, $d_{33}$ can also be recorded, as shown in Fig. 28(b). The $d_{33}$ value at $E=0 \mathrm{kV} \mathrm{cm}{ }^{-1}$ is the one we record. Therefore, this method will apply an electric field on samples before each measurement, which is totally different from the first method that only needs to be poled for once. For a given poled KNN-based ceramic, increasing temperature will inevitably destroy the ordered domain structure, resulting in the reduced $d_{33}$ without considering the phase transitions. Thus, the repeated application of an electric field during the heating process will compensate for the destruction of the ordered domain structure, resulting in a slow decrease of $d_{33}$. Moreover, the higher the applied electric field, the more the compensation. As expected, Li et al. observed the improved temperature stability of $d_{33}$ by increasing the measuring electric field, as shown in Fig. 28(c). ${ }^{207}$

As discussed in Fig. 24, the strain properties of poled $\mathrm{KNN}$ based ceramics mainly originated from the intrinsic contribution that is closely related to the phase structure. Therefore, temperaturedependent strain properties should be strongly close to the phase structure, as demonstrated in Fig. 28(d)-(i). KNNS-SZ$x$ BNZ ceramics with $x=0.03$ and 0.04 exhibited improved roomtemperature strain properties and temperature stability when compared with those of the ones with $x=0 .{ }^{66}$ We compared the variations of permittivity and $S_{\text {uni(T) }} / S_{\text {uni(RT) }}$ with temperature (see Fig. 28(g)-(i)). Clearly, $S_{\text {uni(T) }} / S_{\text {uni(RT) }}$ increased when the temperature approached $T_{\mathrm{O}-\mathrm{T}}$ (see Fig. $28(\mathrm{~g})$ and $(\mathrm{h})$ ), but sharply reduced as the temperature further increased close to $T_{\mathrm{c}}$ (see Fig. 28(i)), resulting in the similar variations of permittivity and $S_{\text {uni(T) }} / S_{\text {uni(RT) }}$ with temperature. Thus, for KNN-based ceramics, the obtained temperature-dependent strain properties by this method were closely related to the phase structure and the amplitude of the electric field. That is the reason why the most reported enhancement of temperature stability of KNNbased ceramics was achieved by applying a high electric field (e.g., $E \geq 30 \mathrm{kV} \mathrm{cm}^{-1}$ ). In addition, the previous explanations of temperature-dependent $S_{\text {uni }}$ of KNN-based ceramics using domain structure should be reconsidered carefully. ${ }^{62,103}$

Here, we compared the variations of in situ temperaturedependent $S_{\text {uni }}$ among different representative piezoelectric ceramics, as shown in Fig. 29. $S_{\text {uni }}$ of commercial soft PZT-5H and PZT-4 ceramics increased monotonously with increasing temperature. ${ }^{70}$ $S_{\text {uni }}$ of non-textured LF4 ceramics, ${ }^{22}$ BNT-BT-KNN ceramics, ${ }^{208}$ and BT-based ceramics ${ }^{197}$ displayed a strong temperature dependence, that is, $S_{\text {uni }}$ rapidly reduced with increasing temperature. It was 


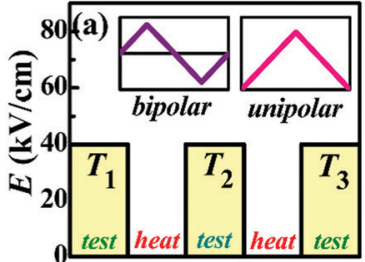

Temp. $\left({ }^{\circ} \mathrm{C}\right)$
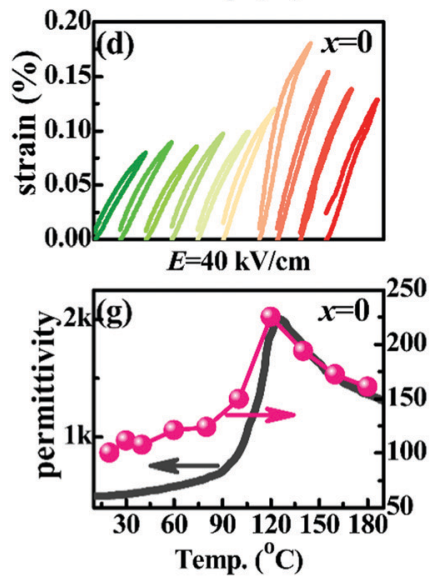
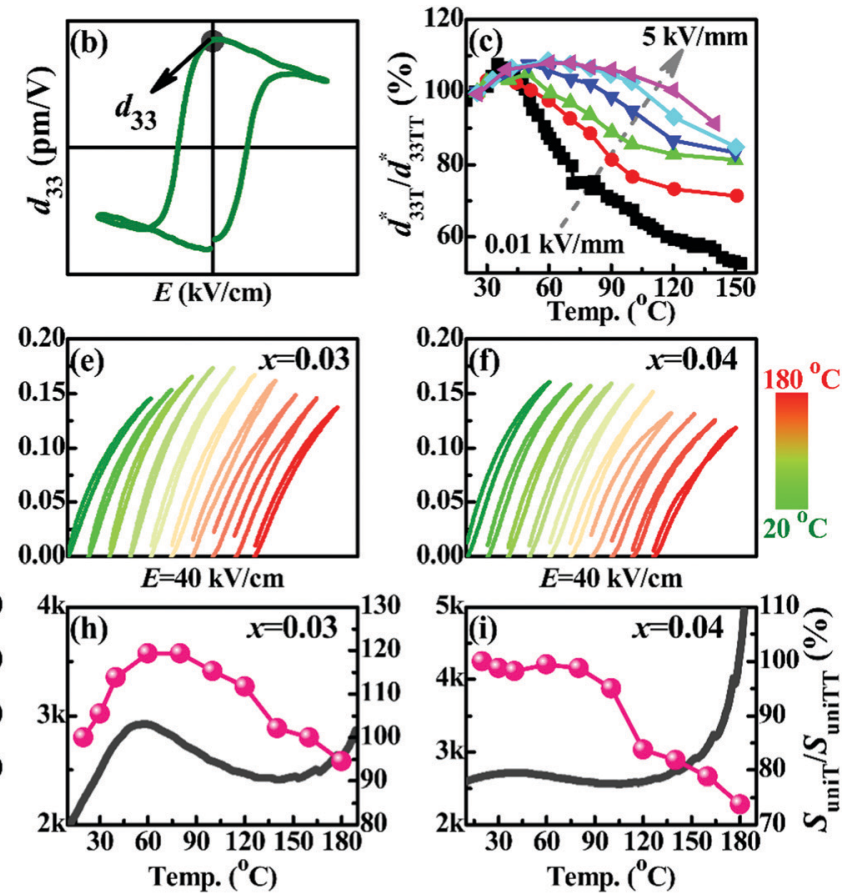

Fig. 28 Temperature-dependent strain properties of KNN-based ceramics. (a) The schematic diagram for measuring temperature-dependent bipolar and unipolar strain curves. (b) $d_{33}$ curve during the bipolar measurement. (c) Temperature-dependent $d_{33 T^{*}} / d_{33 R T}{ }^{*}$ value of KNNLT-CZ5 ceramics under different electric fields. Temperature-dependent unipolar strain curves, temperature-dependent permittivity and $S_{\text {uni(T) }} / S_{\text {uni(RT) }}$ value of KNNS-SZ-xBNZ ceramics with $x=0$ ( $\mathrm{d}$ and g), $x=0.03$ (e and h), and $x=0.04$ ( $f$ and i). (c) Reproduced from ref. 207 with permission from Wiley-VCH, Copyright 2016; (d-i) reproduced from ref. 66 with permission from Wiley-VCH, Copyright 2018.

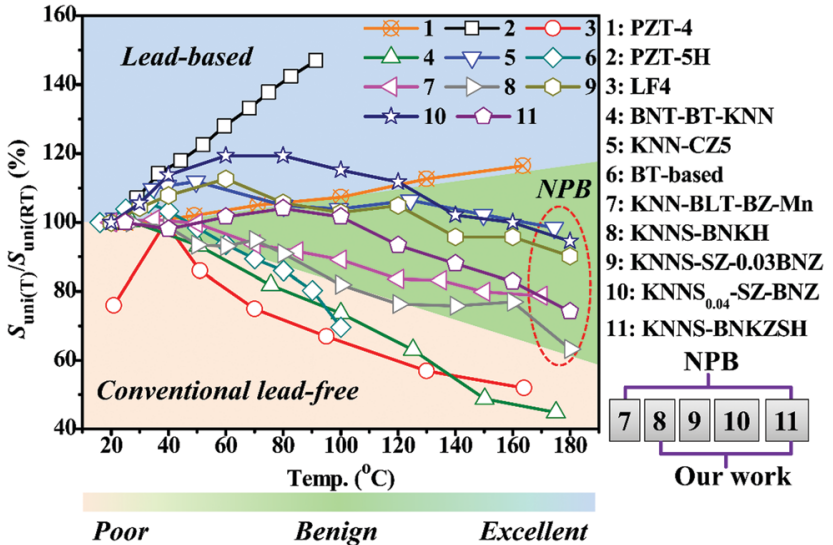

Fig. 29 Comparison of $S_{\text {uni(T) }} / S_{\text {uni(RT) }}$ among different representative piezoelectric ceramics.

worth noting that KNN-based ceramics with NPB exhibited a temperature stability between that of PZT-5H ceramics and aforementioned lead-free ceramics. ${ }^{25,27,31,66,70,143}$ It was also found that the temperature stability of KNN-based ceramics with NPB was strongly dependent on the composition design, indicating that a more elaborate composition design would obtain better temperature stability. Therefore, the temperature stability of piezoceramics' strain properties can be divided into three areas, as shown in Fig. 29. KNN-based ceramics without phase boundaries and BT-based ceramics occupied the bottom left area showing poor temperature stability. Lead-based ceramics represented by PZT-5H and PZT-4 ceramics shared an area with excellent temperature stability. On the other hand, KNN-based ceramics with NPB filled up the gap of temperature stability between the commercial lead-based ceramics and the conventional lead-free ceramics, taking up an area with benign temperature stability. Particularly, KNNS-SZ-BNZ ceramics even showed comparable temperature stability to that of PZT- 4 ceramics in the temperature range of $20-120{ }^{\circ} \mathrm{C}$, which was the general operating temperature for piezoelectric actuators. ${ }^{66,143}$ In conclusion, the construction of the NPB not only increased the room-temperature strain properties of KNN-based ceramics but also relieved its temperature dependence, which endowed the KNN-based ceramics with NPB with promising potential for industrial applications.

Annealing in a muffle furnace. This method is to heat the poled samples at a fixed temperature point for $0.5-1 \mathrm{~h}$ and then measure their $d_{33}$ values at room temperature, as simulated in Fig. 30(a). Therefore, this method is actually to measure the resistibility of poled KNN-based ceramics' piezoelectric properties against thermal impact (also called as thermal stability). Lead-based ceramics with MPB exhibited a stable $d_{33}$ value before $T_{\mathrm{c}}$ as expected (see Fig. 30(b)). ${ }^{209}$ On the other hand, $d_{33}$ values of KNNS- $x$ BNZH (e.g., $x=0$ for $\mathrm{O}$ phase and $x=0.0375$ for NPB) ceramics first decreased slightly at $T \ll T_{\mathrm{c}}$, and then sharply reduced at $T-T_{\mathrm{c}}$ (see Fig. 30(c) and (d)). ${ }^{136}$ From the viewpoint of this method, the NPB can increase the initial $d_{33}$ value without significantly sacrificing thermal stability. Unfortunately, this method cannot 

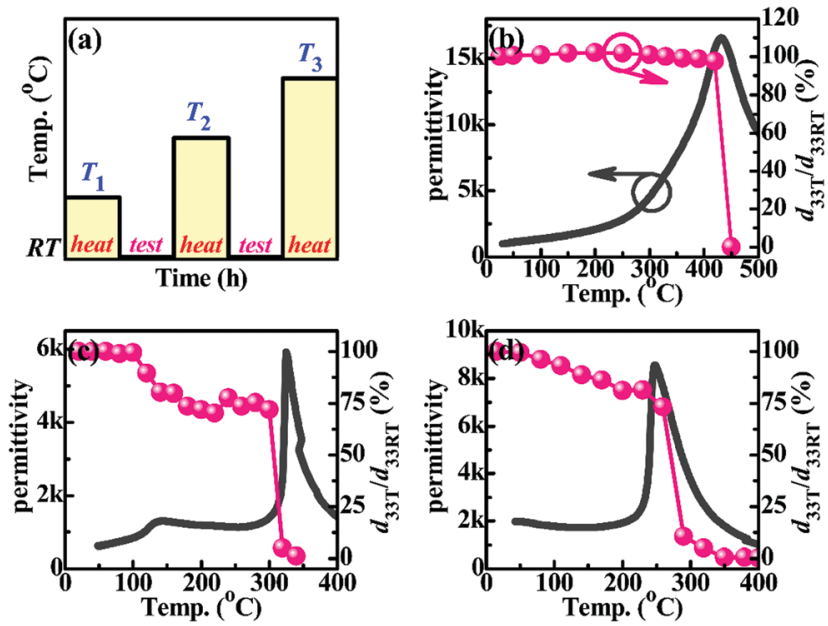

Fig. 30 Annealing behavior of KNN-based and lead-based ceramics (a) The schematic diagram for measuring the annealing behavior of poled piezoceramics. Temperature-dependent permittivity and $d_{33}$ of $0.99\left(0.36 \mathrm{BiScO}_{3}-0.64 \mathrm{PbTiO}_{3}\right)-0.01 \mathrm{Bi}\left(\mathrm{Zn}_{0.5} \mathrm{Ti}_{0.5}\right) \mathrm{O}_{3}$ lead-based (b) and KNNS $-x$ BNZH ceramics with $x=0$ (c) and $x=0.0375$ (d). (b) Reproduced from ref. 209 with permission from Elsevier, Copyright 2018; (c and d) reproduced from ref. 136 with permission from Elsevier, Copyright 2016.

truly reflect the $d_{33}$ values of KNN-based ceramics at an ambient temperature like that of the first method.

3.3.2 Way to improve temperature stability. As discussed above, different methods were used to examine the temperature stability of KNN-based ceramics. The obvious temperature dependence of piezoelectric properties fundamentally originates from the trait of PPB that is closely related to both ambient temperature and composition design. Although increasing the measurement electric field could result in improved temperature stability of strain properties, the industrial applications could not offer a high electric field. Considering the important role of temperature stability in practical applications, the researchers have tried out different approaches to improve the temperature stability without significantly sacrificing the piezoelectric properties, such as optimizing the preparation technology, ${ }^{210-212}$ constructing a broadened NPB, ${ }^{62}$ addition of metallic oxides (e.g., $\left.\mathrm{MnO}_{2}\right),{ }^{27,29}$ and fabricating composite ceramics using high-performance $\mathrm{KNN}$ based ceramic matrix and enhancer. ${ }^{63} \mathrm{Li}$ et al. proposed to construct a broadened phase boundary to simultaneously guarantee the piezoelectric properties and temperature stability, ${ }^{62}$ as shown in Fig. 31. They obtained a broadened NPB in a welldesigned composition of $0.925\left(\mathrm{Li}_{x} \mathrm{Na}_{0.53} \mathrm{~K}_{0.48-x}\right) \mathrm{NbO}_{3}-0.065 \mathrm{BaZrO}_{3}-$ $0.01\left(\mathrm{Bi}_{0.5} \mathrm{Na}_{0.5}\right) \mathrm{TiO}_{3}$, whose $d_{33}$ values varied within $-10 \%$ in the common application temperature range $\left(25-100{ }^{\circ} \mathrm{C}\right) .{ }^{62}$ In addition, they recently also obtained the high piezoelectric properties and improved temperature stability of $0.93\left(\mathrm{Li}_{x} \mathrm{Na}_{0.52} \mathrm{~K}_{0.48-x}\right)\left(\mathrm{Nb}_{1-y} \mathrm{Sb}_{y}\right)$ $\mathrm{O}_{3}-0.05 \mathrm{BaZrO}_{3}-0.02\left(\mathrm{Bi}_{0.5} \mathrm{Na}_{0.5}\right) \mathrm{HfO}_{3}-\mathrm{MnO}_{2}\left(\mathrm{~L}_{x} \mathrm{KNNS}_{y}-5 \mathrm{BZ}-2 \mathrm{BNH}-\right.$ $\mathrm{Mn}$ ) ceramics by simultaneously utilizing the structural flexibility and multi-phase coexistence. ${ }^{213}$ The structural flexibility was achieved by the B-site ion substitution, that is, replacing $\mathrm{Nb}^{5+}$ with $\mathrm{Sb}^{5+213}$ The structural flexibility featured multi-phase coexistence exhibited the broadened dielectric anomaly peak near room temperature, which thus can be deemed as a

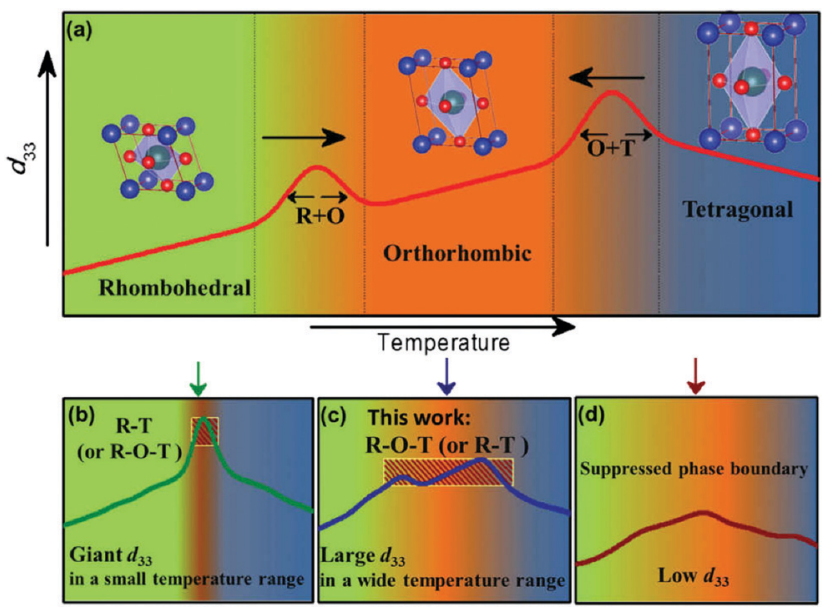

Fig. 31 Schematic diagram of property enhancement by phase boundary engineering. (a) Temperature-dependent permittivity curve of KNN ceramics. (b-d) Possible phase boundary constructed by phase boundary engineering, that is, the sharp phase boundary (b), broadened phase boundary (c), and suppressed phase boundary (d). Reproduced from ref. 62 with permission from the Royal Society of Chemistry, Copyright 2018.

broadened phase boundary to some extent. Therefore, constructing a broadened NPB could achieve a benign balance between piezoelectric properties and temperature stability, ${ }^{62}$ which is one of the future topics of KNN-based ceramics.

Inspired by the success of composites in enhancing the performance of materials and the improved temperature stability of BNT-6BT:ZnO composite ceramics, ${ }^{214}$ our group designed a composite ceramic by using high-performance $0.96\left(\mathrm{~K}_{0.44} \mathrm{Na}_{0.56}\right)$ $\left(\mathrm{Nb}_{0.95} \mathrm{Sb}_{0.05}\right) \mathrm{O}_{3}-0.04 \mathrm{Bi}_{0.5}\left(\mathrm{Na}_{0.18} \mathrm{~K}_{0.82}\right)_{0.5} \mathrm{ZrO}_{3}$ (KNNS-BNKZ) as the ferroelectric matrix and polar $\mathrm{ZnO}$ particles as the enhancer, i.e., KNNS-BNKZ:ZnO composite ceramic. ${ }^{63}$ The CBED patterns demonstrated that the ferroelectric matrix still possessed an R-T phase coexistence, while the enhancer was the p-type Sb-modified ZnO particle (see Fig. 32(a)-(j)). ${ }^{63}$ The composite ceramic exhibited enhanced temperature stability, i.e., $S_{\text {uni }}$ varied within $-16 \%$ to $+20 \%$ in the temperature range of $20-200{ }^{\circ} \mathrm{C}$ (see Fig. $32(\mathrm{k})$ ). ${ }^{63}$ The obtained temperature stability was slightly superior to those of other representative KNN-based ceramics (see Fig. 32(k)). ${ }^{63}$ The analysis showed that the compensatory electric field generated by the Sb-doped $\mathrm{ZnO}$ sub-micron particles was responsible for the improved temperature stability. ${ }^{63}$ More importantly, the composite ceramic also possessed a high $d_{33}$ value of $480-$ 510 pC N ${ }^{-1}$ and an increased $T_{\mathrm{c}}$ value (see Fig. 32(l)). ${ }^{63}$ These high $d_{33}$ values and increased $T_{\mathrm{c}}$ values could fill the gap of the piezoelectric properties between $\mathrm{O}-\mathrm{T}$ phase boundary and NPB (see Fig. 32(1)), providing more options for practical applications. ${ }^{63}$ Therefore, the composite ceramic is another promising way to simultaneously improve the piezoelectric properties and the temperature stability of KNN-based ceramics with NPB. ${ }^{63}$

In addition to elaborately controlling the NPB, the metallic oxide, $\mathrm{MnO}_{2}$, was also reported to further improve the piezoelectric properties and temperature stability. ${ }^{27,29} \mathrm{Li}$ et al. prepared KNNSBLT-BZ- $x \mathrm{Mn}$ ceramics by doping $\mathrm{MnO}_{2}$ into KNN-BLT-BZ ceramics with NPB. ${ }^{27}$ The results showed that the appropriate 

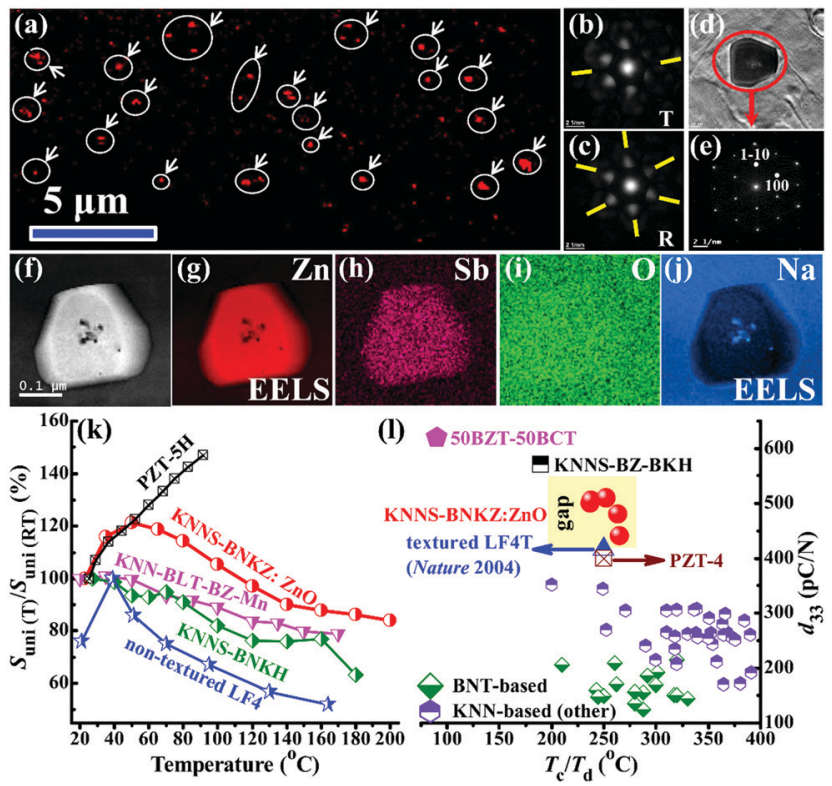

Fig. 32 Microstructure, temperature stability and piezoelectric properties of KNNS-BNKZ:ZnO composite ceramics. (a) The microstructure of KNNS-BNKZ:0.75 wt\% ZnO composite ceramics. The white circles and arrows indicate the location of $\mathrm{ZnO}$ particles. ( $b$ and c) CBED patterns obtained in different areas along the $\langle 111\rangle$ zone axis. (d) $\mathrm{ZnO}$ particle in the KNNS-BNKZ ceramic matrix. (e) Selected area electron diffraction (SAED) pattern along the $\langle 001\rangle$ zone axis of the $\mathrm{ZnO}$ particle shown in (d). (f-j) EDS mapping images of the $\mathrm{ZnO}$ particle. Because the $\mathrm{Zn}(\mathrm{K})$ and $\mathrm{Na}(\mathrm{K})$ peaks overlapped in EDS, the EELS measurement was chosen for distinguishing $Z n$ and Na elements. (k) Comparison of temperature-dependent $S_{\text {uni }}$ of the KNNS-BNKZ:0.75 wt\% ZnO composite ceramic and other representative piezoelectric ceramics, such as commercial soft PIC151 and highperformance KNN-based ceramics with NPB. (l) $d_{33} v s$. $T_{c}\left(T_{d}\right)$ of representative lead-based and lead-free piezoceramics. (a-l) Reproduced from ref. 63 with permission from the American Chemical Society, Copyright 2018.

addition of $\mathrm{MnO}_{2}$ (e.g., $\left.x=1.5\right)$ increased $d_{33}{ }^{*}$ values from $360 \mathrm{pm} \mathrm{V}^{-1}$ to $475 \mathrm{pm} \mathrm{V}^{-1}$ (see Fig. 33(b)). More importantly, KNN-BLT-BZ- $x \mathrm{Mn}(x=1.5)$ ceramics exhibited significantly enhanced temperature stability with respect to KNN-BLT-BZ ceramics (see Fig. 33(a) and (b)). Recently, they also reported the enhanced temperature stability of strain in $\mathrm{KNNS}_{x}-5 \mathrm{BZ}-2 \mathrm{BNH}-$ $1 \mathrm{Mn}$ ceramics. ${ }^{29} \mathrm{KNNS}_{x}-5 \mathrm{BZ}-2 \mathrm{BNH}-1 \mathrm{Mn}(x=0.02-0.025)$ ceramics exhibited the simultaneously enhanced strain properties and temperature stability at a low electric field of $1.5 \mathrm{kV} \mathrm{mm}^{-1}$ (see Fig. 33(c)), which were better than those of other representative leadfree piezoelectric ceramics and even comparable to those of partly commercial PZT-based materials (see Fig. 33(d)). 4,22,25,27,70,214-216 Therefore, the addition of metallic oxide is also another useful way to improve the temperature stability of $\mathrm{KNN}$-based ceramics with NPB.

Based on the above-discussed methods and other methods in the literature, we concluded a general way to obtain the desired piezoelectric properties and temperature stability of KNN-based ceramics, as simulated in Fig. 34. As discussed in Section 3.1.3, the piezoelectric properties of KNN-based ceramics were strongly dependent on the phase structure according to the equation $d_{33}=2 Q \varepsilon_{33} P_{\mathrm{s}} \cdot{ }^{81,91,170}$ First of all, it is thus indispensable
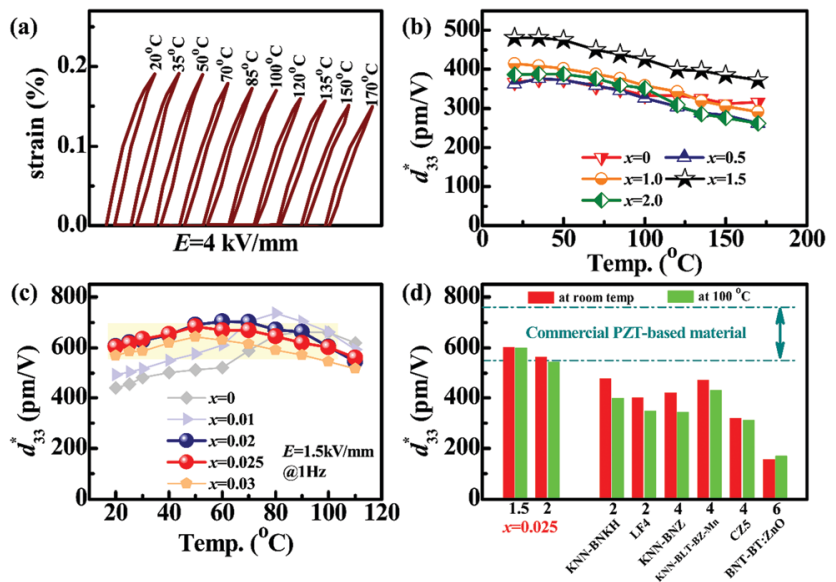

Fig. 33 (a) Temperature-dependent unipolar strain of the KNN-BLT-BZ$x M n(x=1.5)$ ceramic. (b) Temperature-dependent $d_{33^{*}}$ values of the KNN-BLT-BZ-xMn ceramic. (c) Temperature-dependent $d_{33^{*}}$ values of $\mathrm{KNNS}_{x}-5 \mathrm{BZ}-2 \mathrm{BNH}-1 \mathrm{Mn}$ ceramics. (d) Comparison of $d_{33^{*}}$ at room temperature and $100{ }^{\circ} \mathrm{C}$ under certain electric fields of several representative systems. The numbers under the $x$ axis of (d) show the amplitude of the electric field with the unit of $\mathrm{kV} \mathrm{mm} \mathrm{m}^{-1}$. ( $\mathrm{a}$ and $\mathrm{b}$ ) Reproduced from ref. 27 with permission from the American Chemical Society, Copyright 2017; (c and d) reproduced from ref. 29 with permission from the Royal Society of Chemistry, Copyright 2018.

for KNN-based ceramics to construct a phase boundary (particularly NPB) because of the significant piezoelectricity enhancement at the NPB. ${ }^{10,23,25-28,44}$ Subsequently, different methods can be adopted to further modify the KNN-based with NPB, such as optimizing preparation technology, ${ }^{210-212}$ elaborately controlling the phase boundary, ${ }^{31,62}$ addition of metallic oxides (e.g., $\left.\mathrm{MnO}_{2}\right),{ }^{27,29}$ and composite ceramic. ${ }^{63}$ And then, these methods will affect the KNN-based ceramics with NPB with regard to different factors (e.g., phase structure, microstructure, and external compensation) (see Fig. 34). Finally, the desired piezoelectric properties and temperature stability can be obtained after successfully satisfying these factors.

\section{Validity and scope of the NPB}

Although the NPB was initially proposed by our group, its validity has also been verified by other researchers. Here, we collected the historical evolutions of $d_{33}$ values at the NPB, which were reported by different groups (see Fig. 35). Three stages were clearly observed, marked as stage I, stage II, and stage III. In stage I (2013-2014), our group initially proposed the NPB, by which the large $d_{33}$ values of $425-490 \mathrm{pC} \mathrm{N}^{-1}$ were achieved. ${ }^{42,44,45}$ Therefore, stage I represented the initiation of the NPB. In stage II (20152016), our group obtained the higher $d_{33}$ values of $419-570 \mathrm{pC} \mathrm{N}^{-1}$ by further optimizing the composition design at the NPB. ${ }^{23,26,28,54}$ Meanwhile, other researchers started to construct the NPB with the purpose of achieving high $d_{33}$ values. For example, Fernando et al. reported an enhanced $d_{33}$ value of $400 \mathrm{pC} \mathrm{N}^{-1}$ in KNNLS-0.04BZ ceramics that possessed an R-T phase coexistence. ${ }^{217}$ Guo et al. and Lee et al. also constructed an R-T phase coexistence in KNNLS0.05CBNZ and KNN-BNKLZ-0.015BNT ceramics, respectively, 


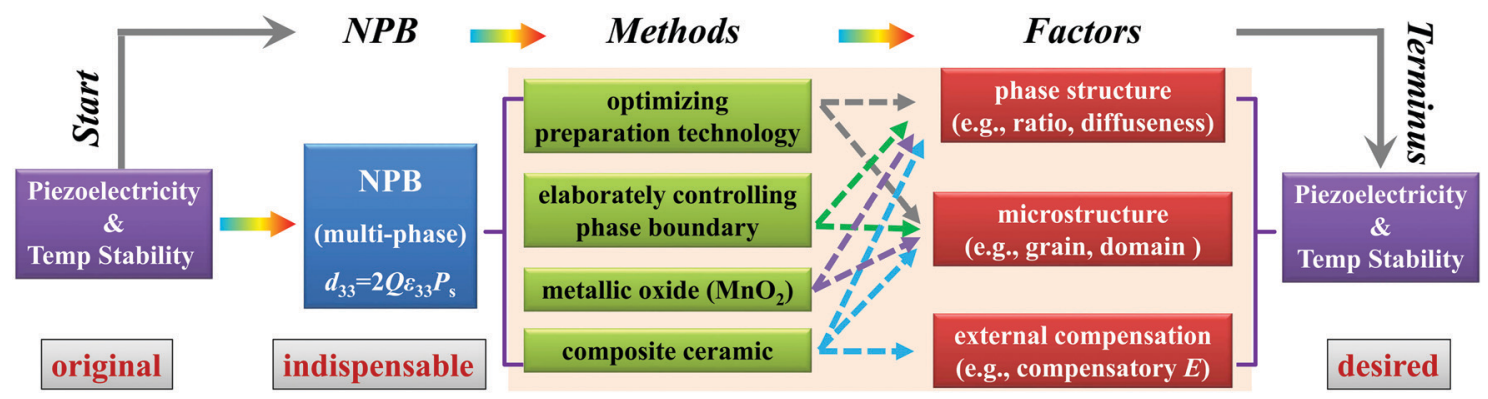

Fig. 34 Way to obtain the desired piezoelectricity and temperature stability for KNN-based ceramics.

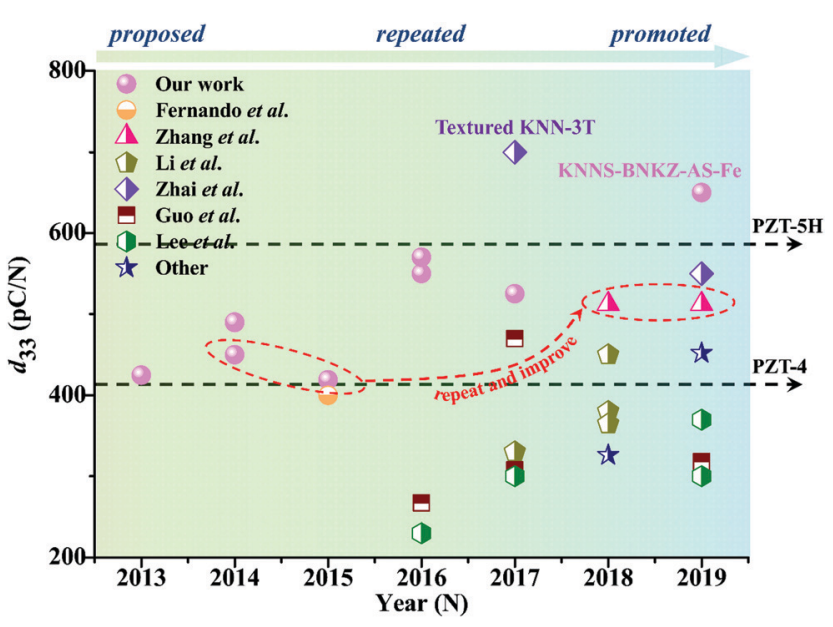

Fig. $35 d_{33}$ values at the NPB reported by different groups as a function of year. The data encircled by the red dashed line show the $d_{33}$ values of KNNS-BNZ and KNNS-BNH ceramics reported by our group $(2014,2015)$ and Zhang et al. (2018, 2019).

which exhibited moderate $d_{33}$ values of 230-267 $\mathrm{pC} \mathrm{N}^{-1} \cdot{ }^{218,219}$ Therefore, not only our group but also other researchers can repeat the construction of the NPB, regardless of the relatively moderate $d_{33}$ values reported by others. ${ }^{218-220}$ Stage II was the repetition of the construction or verification of the NPB.

In stage III (2017-now), our group reported better $d_{33}$ values as high as $525-650 \mathrm{pC} \mathrm{N}^{-1}$ by further controlling the NPB by approaches such as the slush nano-polar state method. ${ }^{24,25}$ More importantly, other groups not only constructed the NPB successfully, but also achieved enhanced performance (including $d_{33}$ values and strain temperature stability). ${ }^{73,86,212,221-228}$ For example, Zhang et al. successfully constructed the NPB and even obtained higher $d_{33}$ values (e.g., $512 \mathrm{pC} \mathrm{N}^{-1}$ ) than our previously reported ones (e.g., 419-450 $\mathrm{pC} \mathrm{N}^{-1}$ ), using the same additives (e.g., $\mathrm{Sb}^{5+},\left(\mathrm{Bi}_{0.5} \mathrm{Na}_{0.5}\right) \mathrm{ZrO}_{3}$ and $\left.\left(\mathrm{Bi}_{0.5} \mathrm{Na}_{0.5}\right) \mathrm{HfO}_{3}\right)$, as marked by the red dashed line. ${ }^{45,54,68,69,104}$ By means of constructing the NPB, Li et al. simultaneously achieved enhanced piezoelectric properties $\left(d_{33}=330-450 \mathrm{pC} \mathrm{N}^{-1}\right)$ and temperature stability. ${ }^{27,29,46,62,103}$ Guo et al. and Lee et al. also reported the enhanced $d_{33}$ values of 300$470 \mathrm{pC} \mathrm{N}^{-1}$ at the NPB. ${ }^{221-223,226,227}$ More interestingly, Zhai et al. achieved an ultra-high $d_{33}$ value of $700 \mathrm{pC} \mathrm{N}^{-1}$ in highly textured $\mathrm{KNN}-3 \mathrm{~T}$ ceramics. ${ }^{86}$ Although the textured $\mathrm{KNN}-3 \mathrm{~T}$ ceramics were reported to exhibit an $\mathrm{R}-\mathrm{O}$ phase coexistence, the exact formula of $0.96\left(\mathrm{~K}_{0.5} \mathrm{Na}_{0.5}\right)\left(\mathrm{Nb}_{0.965} \mathrm{Sb}_{0.035}\right)-0.01 \mathrm{CaZrO}_{3}-0.03\left(\mathrm{Bi}_{0.5} \mathrm{~K}_{0.5}\right) \mathrm{HfO}_{3}$ was highly similar to those possessing the NPB. ${ }^{86}$ The addition of $\mathrm{Sb}^{5+}, \mathrm{CaZrO}_{3}$, and $\left(\mathrm{Bi}_{0.5} \mathrm{~K}_{0.5}\right) \mathrm{HfO}_{3}$ would increase $T_{\mathrm{R}-\mathrm{O}}$ and reduce $T_{\mathrm{O}-\mathrm{T}}$, which was what they also observed in $\mathrm{KNN}-3 \mathrm{~T}$ ceramics. $^{86}$ Thus, they recently also observed an $\mathrm{R}-\mathrm{O}-\mathrm{T}$ phase coexistence in $\langle 00 l\rangle$-textured $0.96\left(\mathrm{~K}_{0.5} \mathrm{Na}_{0.5}\right)\left(\mathrm{Nb}_{0.965} \mathrm{Sb}_{0.035}\right) \mathrm{O}_{3}-$ $0.01 \mathrm{CaZrO}_{3}-0.03\left(\mathrm{Bi}_{0.5} \mathrm{~K}_{0.5}\right) \mathrm{HfO}_{3}$ ceramics that exhibited a high $d_{33}$ value of $550 \mathrm{pC} \mathrm{N} \mathrm{N}^{-1},{ }^{212}$ indicating that the validity of the NPB can be extended to the textured KNN-based ceramics. Therefore, stage III was the promotion of the NPB.

Here, it should also be pointed out that the reported results from different groups may be different. ${ }^{45,54,68,69}$ Moreover, the phase structure and performance were also different even in the same or similar KNN-based ceramics prepared by the same group. ${ }^{86,212}$ This phenomenon widely exists because of the close relationship of "composition design-preparation technologyperformance" in all materials. ${ }^{78,79}$ Even the raw materials with different purity may also affect the structure and performance of KNN-based ceramics. For example, Wang et al. prepared three kinds of pure $\mathrm{KNN}$ ceramics (i.e., $\mathrm{KNN}-\mathrm{O}, \mathrm{KNNS}^{-\mathrm{M}_{1}}$, and $\mathrm{KNN}-\mathrm{M}_{2}$ ) by using $\mathrm{Nb}_{2} \mathrm{O}_{5}$ precursors with different phases (i.e., O phase, $\mathrm{M}_{1}$ phase, and $\mathrm{M}_{2}$ phase). ${ }^{229}$ The results showed that though the $d_{33}$ values of these pure KNN ceramics were highly similar, a significant difference was observed in dielectric properties, ferroelectric properties and strain properties, which was ascribed to the grain growth kinetics of the heterogeneous calcined powder. $^{229}$ Thus, the exact electrical properties of KNN-based ceramics with NPB are also dependent on the composition design and the way to the ceramics are fabricated. Therefore, the higher $d_{33}$ values of $512 \mathrm{pC} \mathrm{N} \mathrm{N}^{-1}$ in KNNS-0.04BNZ and KNNS-0.0375BNH ceramics reported by Zhang et al. may originate from the two-step sintering method. ${ }^{68,69}$ As discussed in Section 3.1.2, the two-step sintering method could further improve the piezoelectric properties of KNN-based ceramics, which was also proved in their previous work. ${ }^{85}$ Unfortunately, few investigations were concentrated on the optimization of the preparation technology of KNN-based ceramics with NPB. Thus, better performance is highly expected if one further optimizes the preparation technology of KNN-based ceramics with NPB.

Encouragingly, the NPB, which was firstly proposed in $\mathrm{KNN}$ based bulk ceramics, is also suitable for KNN-based thin films. ${ }^{230,231}$ It is well-known that KNNS- $x$ BNKZ ceramics are the typical materials for constructing the NPB. ${ }^{44}$ Yao et al. fabricated the KNNS $-x$ BNKZ thin films by using the solution deposition method and measured their effective piezoelectric strain coefficient $\left(d_{33}^{\text {eff }}\right)$ 
(a)

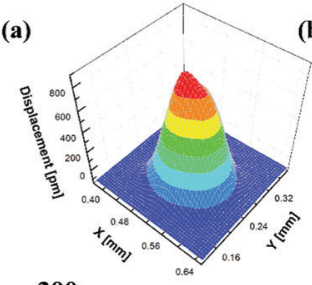

(b)

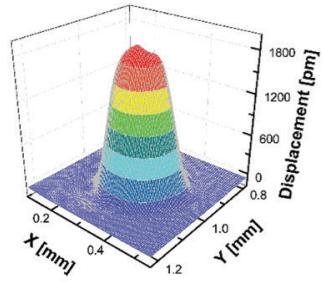

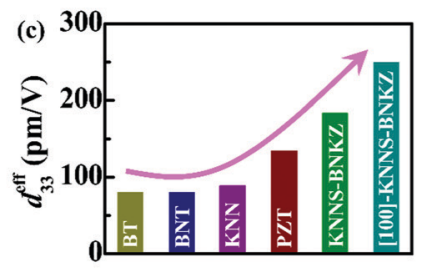
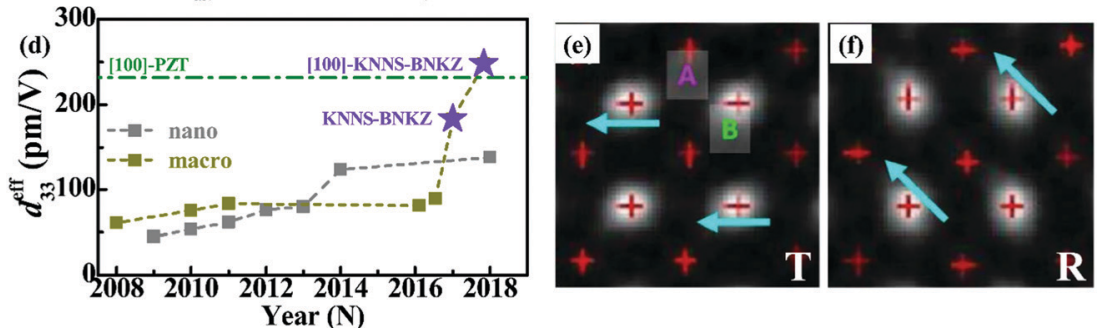

Year $(\mathbf{N})$

Fig. 36 Three-dimensional (3D) drawing of the piezoelectric dilatation for (a) KNSN-0.05BNKZ (with a thickness of $2 \mu \mathrm{m}$ ) and (b) $\langle 100\rangle$-oriented KNNS$0.05 \mathrm{BNKZ}$ (with a thickness of $2.7 \mu \mathrm{m}$ ) thin films, tested under $5 \mathrm{~V}$ at $1.5 \mathrm{kHz}$. (c) The effective piezoelectric strain coefficient ( $d_{33}^{\text {eff }}$ ) values of representative piezoelectric thin films. (d) Historical evolutions of ( $d_{33}^{\text {eff }}$ ) values of KNN-based thin films. (e and f) High-magnification STEM HAADF images of $\langle 100\rangle-$ oriented KNNS-BNKZ thin films. The letters " $A$ " and " $B$ " represent the A and B sites of an $\mathrm{ABO}_{3}$ perovskite unit. (a-f) Reproduced from ref. 230 and 231 with permission from Wiley-VCH, Copyright 2017, 2019

values using a laser scanning vibrometer. ${ }^{230} \mathrm{~A}$ high $d_{33}^{\text {eff }}$ value of $184 \mathrm{pm} \mathrm{V}^{-1}$ was obtained in the KNNS-0.05BNKZ film (see Fig. 36(a)), which was higher than those of other representative lead-free piezoelectric films (see Fig. 36(c)). Then, they further prepared the $\langle 100\rangle$-oriented KNNS- $x$ BNKZ thin films. ${ }^{231}$ The results showed that $\langle 100\rangle$-oriented KNNS-0.05BNKZ ceramics exhibited a higher $d_{33}^{\text {eff }}$ value of $250 \mathrm{pm} \mathrm{V}^{-1}$, which was the highest value in lead-free piezoelectric thin films and was even higher than that of the $\langle 100\rangle$-oriented PZT film (see Fig. 36(d)). ${ }^{230-235}$ In addition, KNNS-0.05BNKZ and $\langle 100\rangle$-oriented KNNS-0.05BNKZ films also further promoted the development of piezoelectric properties in KNN-based films (see Fig. 36(d)). Finally, the phase structure of the $\langle 100\rangle$-oriented KNNS-0.05BNKZ film was identified as an R-T phase coexistence by the STEM HAADF image (see Fig. 36(e) and (f)). Therefore, the enhanced piezoelectric properties were mainly attributed to the multi-phase coexistence that can lower the domain wall energy and facilitate the large piezoelectric response. ${ }^{230,231}$ Therefore, the NPB can also significantly improve the piezoelectric properties of KNN-based films. Considering the extensive applications of piezoelectric films in the micro devices, it is important to carry out further investigations on the NPB in KNN-based films in the future.

Although the NPB was proposed in KNN-based ceramics, it can also be used in other lead-free piezoceramics, which undergo a similar phase transition with temperature as KNN-based ceramics (such as BT-based ceramics). Previously, the researchers mainly concentrated on constructing a multi-phase coexistence point to improve the piezoelectric properties of BT-based ceramics, for example, high $d_{33}$ values of $620 \mathrm{pC} \mathrm{N}^{-1}$ and $697 \mathrm{pC} \mathrm{N}^{-1}$ for a tricritical triple point and a quasi-quadruple point, respectively. ${ }^{19,166}$ But however, the high $d_{33}$ values at the multi-phase coexistence point are metastable, which are sensitive to the variations or fluctuations of composition and temperature. ${ }^{19,166}$ To relieve this sensitivity, our group employed a novel phase-boundary engineering strategy (NPB) utilizing the multiphase convergence, which induced a broad structural flexibility in a wide phase-boundary zone with contiguous polymorphic phase transitions (see Fig. 37(a)). ${ }^{82}$ With the increasing content of BCT, the quasi-quadruple point was gradually opened, manifested by the decreasing $T_{\mathrm{R}-\mathrm{O}}$ and $T_{\mathrm{O}-\mathrm{T}}$ as well as the increasing $T_{\mathrm{c}}$. Obviously, a multi-phase coexistence zone was extended to a composition range of $x=0.05-0.22$ (see Fig. 37(a)). Then, we obtained an ultra-high $d_{33}$ of $700 \pm 30 \mathrm{pC} \mathrm{N}^{-1}$ and high $d_{33}$ values ( $>600 \mathrm{pC} \mathrm{N}^{-1}$ ) over a wide composition range in $\mathrm{BTS}_{0.11^{-}}$ $x$ BCT ceramics (see Fig. 37(b)), the highest value reported so far in non-textured lead-based ceramics (see Fig. 37(c)). The STEM HAADF image directly demonstrated the coexistence of $\mathrm{R}, \mathrm{O}$, and $\mathrm{T}$ phases, as well as the gradual transformation of their polarization vectors (see Fig. 37(d)-(f)). Phase-field simulations proved the low barrier among the three phases, suggesting an easy polarization rotation that was responsible for the enhanced piezoelectric properties (see Fig. 37(g)-(j)). Therefore, the NPB can be extended to BT-based ceramics, which even exhibit better performance (e.g., piezoelectric properties and composition insensitivity) than those of other multi-phase coexistence points. ${ }^{19,82,166}$

\section{Incipient devices}

Considering the high-performance of KNN-based ceramics with $\mathrm{NPB}$, the researchers have tried to apply them for practical applications, such as piezoelectric transducers and piezoelectric nanogenerators. ${ }^{33,36,236}$ For example, Zhu et al. fabricated a piezoelectric transducer by using high-performance $0.96\left(\mathrm{~K}_{0.48^{-}}\right.$ $\left.\mathrm{Na}_{0.52}\right)\left(\mathrm{Nb}_{0.95} \mathrm{Sb}_{0.05}\right) \mathrm{O}_{3}-0.04 \mathrm{Bi}_{0.5}\left(\mathrm{Na}_{0.82} \mathrm{~K}_{0.18}\right)_{0.5} \mathrm{ZrO}_{3}$ (0.96KNNS0.04BNKZ) ceramics with NPB, as shown in Fig. 38(a). ${ }^{236}$ The measurement results suggested that the KNNS-BNKZ transducer exhibited a resonance frequency $\left(f_{\mathrm{r}}\right)$ of $34.2 \mathrm{MHz}$, an antiresonance frequency $\left(f_{\mathrm{a}}\right)$ of $39.8 \mathrm{MHz}$, and a central frequency of $37 \mathrm{MHz}$ (see Fig. 38(b) and (c)). ${ }^{236}$ Then, the calculated results 

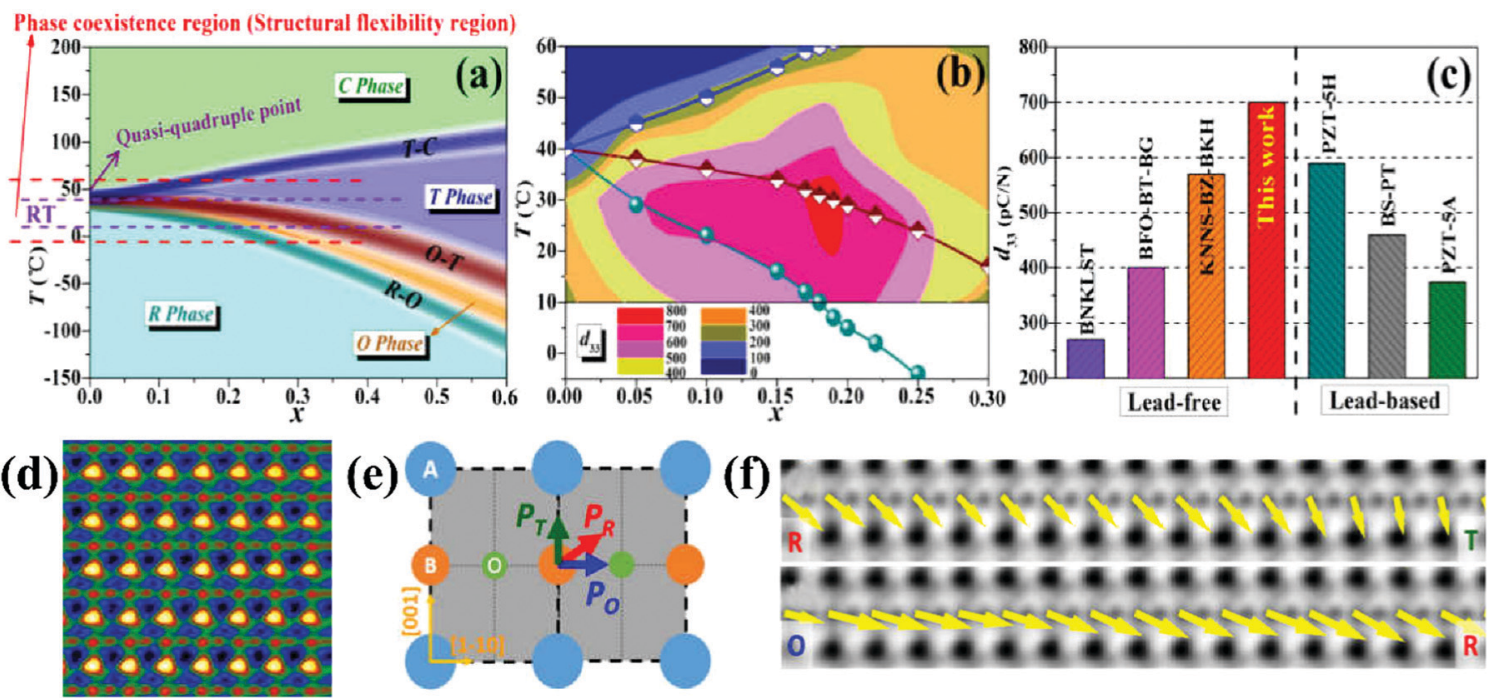

(g)

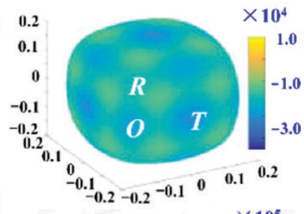

(h)

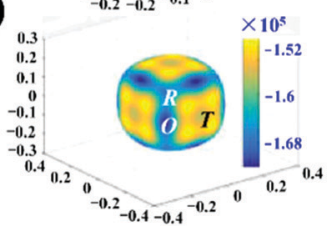

(i)

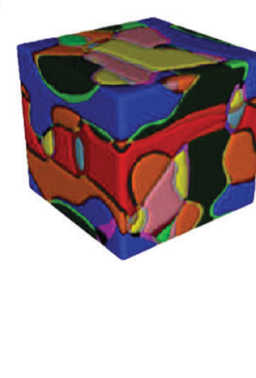

(j)

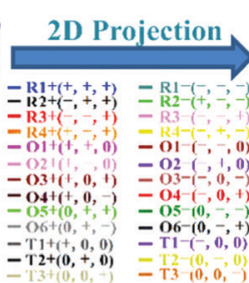

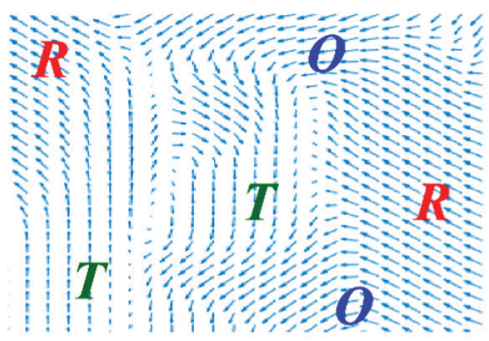

Fig. 37 (a) Phase diagram and (b) temperature-dependent $d_{33}$ values of $\mathrm{BTS}_{0.11}-x \mathrm{BCT}$ ceramics. (c) Comparison of $d_{33}$ values among representative lead-free and lead-based piezoceramics. (d) STEM HAADF image of $\mathrm{BTS}_{0.11}-x \mathrm{BCT}(x=0.18)$ ceramics along the $\langle 110\rangle$ zone axis. (e) Projection of the $\mathrm{ABO}_{3}$-type unit along the $\langle 110\rangle$ zone axis. The directions of the polarization vector of $\mathrm{R}, \mathrm{O}$, and $\mathrm{T}$ phases are $(111)_{\mathrm{pc}},(110)_{\mathrm{pc}}$, and $(001)_{\mathrm{pc}}$, respectively. (f) Enlarged view of the STEM HAADF image showing the transition of polarization vectors among three phases. Free energy profiles of $B T S_{0.11}-x B C T$ ceramics with (g) $x=0$ and (h) $x=0.18$. (i) Phase-field simulations of $\mathrm{T}-\mathrm{O}-\mathrm{R}$ three-phase coexistence in $\mathrm{BTS} \mathrm{S}_{0.11}-x \mathrm{BCT}$ ceramics with $x=0-0.2$ and (j) their projection of polarization on the (110) plane. (a-j) Reproduced from ref. 82 with permission from the American Chemical Society, Copyright 2018.
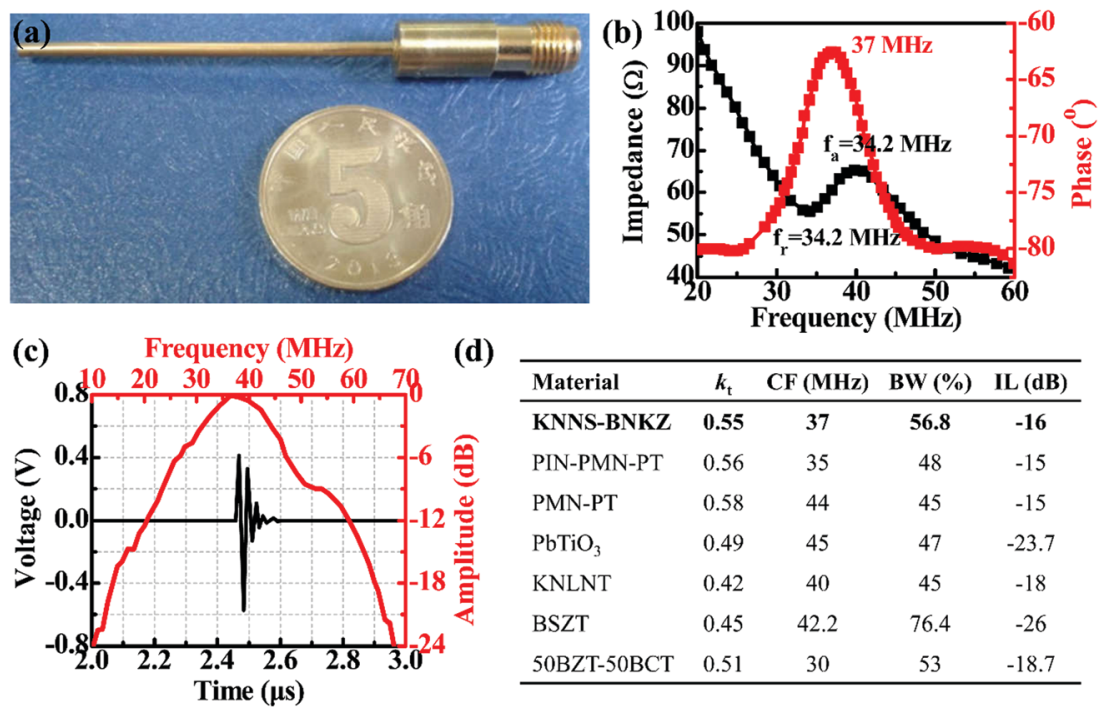

(d)

\begin{tabular}{lcccc}
\hline Material & $\boldsymbol{k}_{\mathbf{t}}$ & $\mathbf{C F}(\mathbf{M H z})$ & $\mathbf{B W}(\%)$ & $\mathrm{IL}(\mathbf{d B})$ \\
\hline KNNS-BNKZ & $\mathbf{0 . 5 5}$ & $\mathbf{3 7}$ & $\mathbf{5 6 . 8}$ & $\mathbf{- 1 6}$ \\
PIN-PMN-PT & 0.56 & 35 & 48 & -15 \\
$\mathrm{PMN}-\mathrm{PT}$ & 0.58 & 44 & 45 & -15 \\
$\mathrm{PbTiO}_{3}$ & 0.49 & 45 & 47 & -23.7 \\
$\mathrm{KNLNT}$ & 0.42 & 40 & 45 & -18 \\
$\mathrm{BSZT}$ & 0.45 & 42.2 & 76.4 & -26 \\
50BZT-50BCT & 0.51 & 30 & 53 & -18.7 \\
\hline
\end{tabular}

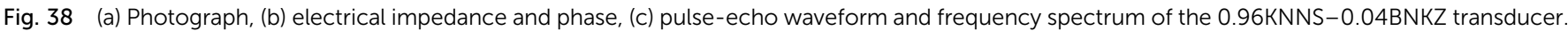

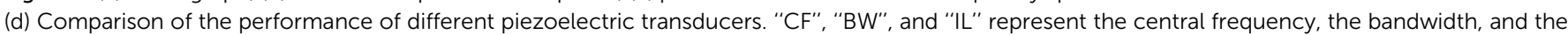
insertion loss, respectively. (a-d) Reproduced from ref. 236 with permission from the Springer Nature Publishing Group, Copyright 2015. 

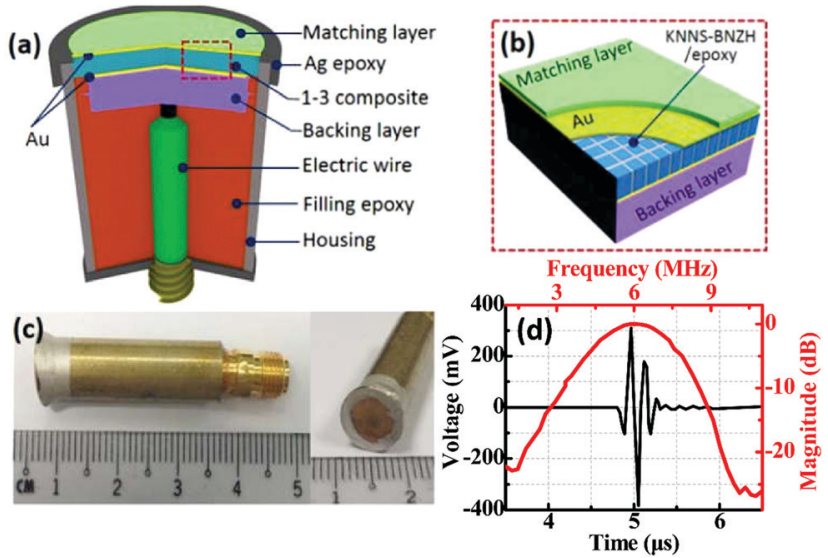

Fig. 39 (a) Schematic structure of a single-element ultrasonic transducer (b) The zoom-in view of the selected area in (a) showing the detailed structure of the transducer. (c) Photographs of an obtained ultrasonic transducer prototype. (d) Pulse-echo waveform and frequency spectrum of the ultrasonic transducer. (a-d) Reproduced from ref. 36 with permission from the Institute of Electrical and Electronics Engineers Inc., Copyright 2019.

showed that the KNNS-BNKZ transducer possessed a thickness mode electromechanical coupling coefficient $\left(k_{t}\right)$ of 0.55 , which was higher than that of other representative lead-free piezoceramics (e.g., 50BZT-50BCT), and even comparable to that of lead-based piezoelectric materials (e.g., PIN-PMN-PT single crystal and $\mathrm{PbTiO}_{3}$ ceramics) (see Fig. 38(d)). ${ }^{236-242}$ Therefore, KNN-based ceramics with NPB have promising potential in piezoelectric transducers.

By using the dice-and-fill method, we and our collaborators successfully fabricated the $0.9625\left(\mathrm{~K}_{0.45} \mathrm{Na}_{0.55}\right)\left(\mathrm{Nb}_{0.96} \mathrm{Sb}_{0.04}\right) \mathrm{O}_{3^{-}}$ $0.0375 \mathrm{Bi}_{0.5} \mathrm{Na}_{0.5} \mathrm{Zr}_{0.85} \mathrm{Hf}_{0.15} \mathrm{O}_{3}$ (KNNS-BNZH)/epoxy 1-3 composite, as shown in Fig. 39 (a)-(d). ${ }^{36}$ The measurement results indicated that the composite exhibited outstanding transducer performance comparable to that of PZT-based transducers, as listed in Table $4 .{ }^{36}$ First of all, the transducer performance was improved when changing the phase structure of KNN-based ceramics from a single $\mathrm{O}$ phase to multiphase coexistence (e.g., $\mathrm{O}-\mathrm{T}$ and $\mathrm{NPB}$ ). $d_{33}$ and $k_{\mathrm{t}}$ values were significantly increased

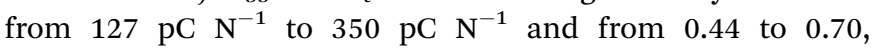
respectively. The KNN-based transducers with NPB exhibited better performance than those with $\mathrm{O}-\mathrm{T}$ phase boundary. Finally, the KNN-based transducers with NPB (e.g., KNNSBNZH/epoxy 1-3 composite) displayed comparable performance to that of PZT-based transducers (e.g., PZT-5H and
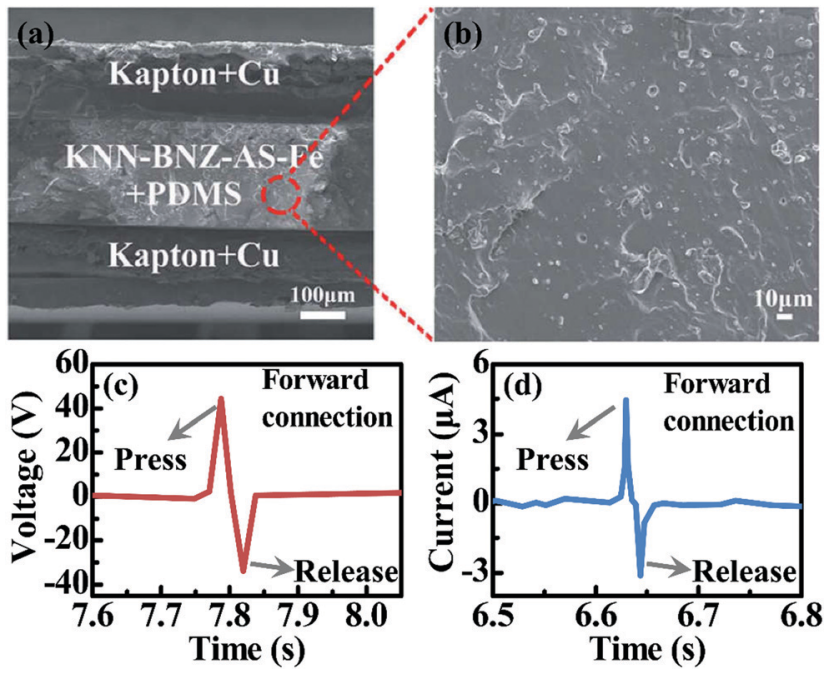

Fig. 40 Structure and performance of KNN-BNZ-AS-Fe piezoelectric nanogenerators (PENG). (a and b) Cross-sectional SEM images of the KNN-BNZ-AS-Fe flexible PENG device. Piezoelectric output (c) voltage and (d) current of the KNN-BNZ-AS-Fe flexible PENG device in the forward connection during one cycle. $(a-d)$ Reproduced from ref. 33 with permission from the Royal Society of Chemistry, Copyright 2018.

PZT/epoxy 1-3 composite). Therefore, the KNN-based ceramic with NPB can serve as a promising alternative to lead-based piezoelectric materials for ultrasonic transducer applications. ${ }^{36}$

Zheng et al. prepared a piezoelectric nanogenerator (PENG) by using $0.91 \mathrm{~K}_{0.48} \mathrm{Na}_{0.52} \mathrm{NbO}_{3}-0.04 \mathrm{Bi}_{0.5} \mathrm{Na}_{0.5} \mathrm{ZrO}_{3}-0.05 \mathrm{AgSbO}_{3}-$ $0.2 \% \mathrm{Fe}_{2} \mathrm{O}_{3}(\mathrm{KNN}-\mathrm{BNZ}-\mathrm{AS}-\mathrm{Fe})$ ceramic particles and a polydimethylsiloxane (PDMS) matrix, ${ }^{33}$ as shown in Fig. 40(a) and (b). KNN-BNZ-AS-Fe ceramics possessed the NPB as well as an ultra-high $d_{33}$ value of $500 \mathrm{pC} \mathrm{N}^{-1} .^{33}$ The measurement results showed that the PENG exhibited an optimized output voltage of $52 \mathrm{~V}$ and a current of $4.8 \mu \mathrm{A}$ under a vertical force of $25 \mathrm{~N}$ at $2 \mathrm{~Hz}$ (see Fig. 40(c) and (d)). ${ }^{33}$ In addition, the PENG also exhibited a benign fatigue resistance, accompanied by a slightly reduced output voltage after 14 days. Therefore, KNN-based ceramics with NPB are promising for piezoelectric nanogenerator applications. ${ }^{33}$ Up to now, KNN-based ceramics with NPB were rarely reported in the fabrication of incipient devices in spite of their excellent performance. Therefore, it will be more interesting and urgent to see the performance of $\mathrm{KNN}$-based ceramics with NPB in different devices. ${ }^{33}$

In addition to the traditional electronic devices, KNN-based ceramics with NPB also have promising potential in other

Table 4 Comparison of the transducer characteristics and performances obtained by different piezoelectric ceramics

\begin{tabular}{|c|c|c|c|c|c|c|c|c|c|}
\hline Materials & Phase boundary & $d_{33}\left(\mathrm{pC} \mathrm{N}^{-1}\right)$ & $\varepsilon_{33}$ & $Z$ (MRayls) & $Q_{\mathrm{m}}$ & $k_{\mathrm{t}}$ & $f_{\mathrm{c}}(\mathrm{MHz})$ & $V_{\mathrm{p}-\mathrm{p}}(\mathrm{V})$ & $\mathrm{BW}_{\mathrm{r}}(\%)$ \\
\hline $\mathrm{KNN}^{243}$ & $\mathrm{O}$ & 127 & 125 & 23 & - & 0.44 & 5.5 & 0.80 & 49 \\
\hline $\mathrm{KNN}-\mathrm{Sr}^{244}$ & $\mathrm{O}$ & - & 275 & 24.6 & - & 0.35 & 7 & 0.14 & 140 \\
\hline $\mathrm{KNN}-\mathrm{Ba}^{245}$ & $\mathrm{O}$ & 210 & 1173 & 26.7 & 72 & 0.37 & 5.5 & - & 50 \\
\hline $\mathrm{KNN}-\mathrm{Li}, \mathrm{Sb}^{246}$ & $\mathrm{O}-\mathrm{T}$ & 305 & 1600 & - & 40 & 0.51 & 3.2 & 0.80 & 55 \\
\hline KNN-Li,Ta/epoxy 1-3 composite com $^{247}$ & $\mathrm{O}-\mathrm{T}$ & 140 & 302 & 6.6 & 18 & 0.65 & 29 & 0.14 & 90 \\
\hline $\mathrm{PZT}-5 \mathrm{H}^{36}$ & MPB & 595 & 2000 & 33 & - & 0.49 & 5 & - & 58 \\
\hline PZT/epoxy 1-3 composite coms $^{248}$ & MPB & 374 & 3400 & 13.4 & - & 0.59 & 5 & - & 73 \\
\hline KNNS-BNZH/epoxy 1-3 composite com $^{36}$ & NPB & 350 & 1342 & 15.8 & 5 & 0.70 & 5 & 0.78 & 80 \\
\hline
\end{tabular}



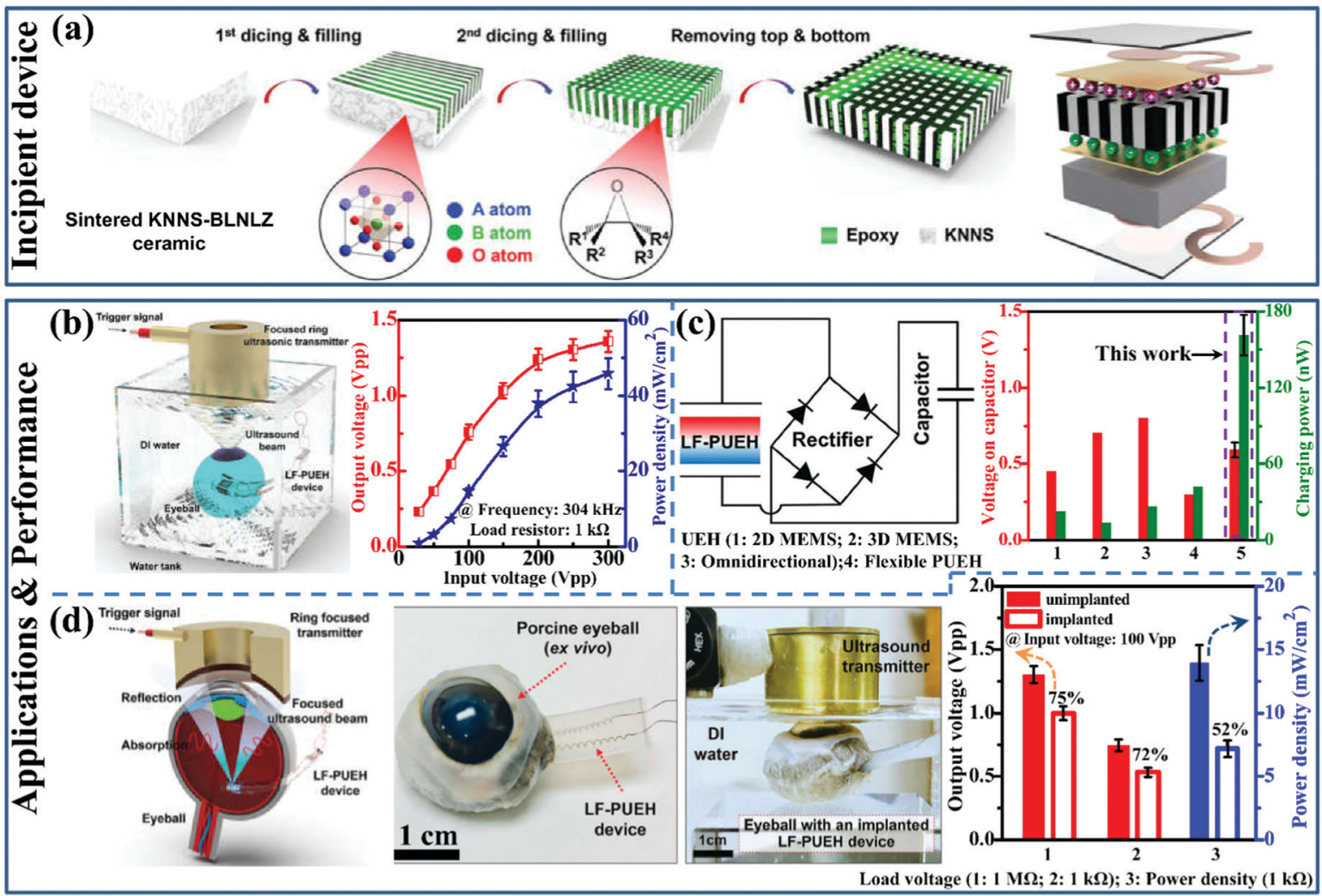

Fig. 41 (a) Fabrication process of the 1-3 piezocomposite component using a modified dice-and-fill technique. (b) Test setup and electrical characterization of the LF-PUEH device. (c) Applications of the LF-PUEH device on microelectronic devices and the comparison of the average charging power of different UEHs. (d) Evaluation of the LF-PUEH device in the ex vivo experiment of an implanted environment. (a-d) Reproduced from ref. 35 with permission from Wiley-VCH, Copyright 2019.

fields, such as medical instruments. It is feasible to restore vision in people with neurodegenerative diseases by using retinal electrical stimulation for direct excitation of neurons. ${ }^{35}$ In this context, Zhou et al. proposed a new electrical stimulation strategy by using ultrasound-driven wireless energy harvesting technology that can convert acoustic energy to electricity through the piezoelectric effect. ${ }^{35}$ Firstly, they fabricated the 1-3 piezocomposite component comprised of high-performance $0.96\left(\mathrm{~K}_{0.48^{-}}\right.$ $\left.\mathrm{Na}_{0.52}\right)\left(\mathrm{Nb}_{0.95} \mathrm{Sb}_{0.05}\right) \mathrm{O}_{3}-0.04\left(\mathrm{Bi}_{0.4} \mathrm{La}_{0.1}\right)\left(\mathrm{Na}_{0.4} \mathrm{Li}_{0.1}\right) \mathrm{ZrO}_{3}$ (KNNS-BLNLZ) ceramics with NPB and insulating epoxy by using a modified dice-and-fill method (see Fig. 41(a)). And then, the lead-free ultrasonic energy harvester (LF-UEH) was prepared using the 1-3 piezocomposite component (see Fig. 41(a)) and it exhibited benign mechanical flexibility. Subsequently, the electrical properties of LF-UEH were measured. The LF-UEH can be driven by ultrasound to produce adjustable electrical outputs, reaching a maximum output power of $45 \mathrm{~mW} \mathrm{~cm}{ }^{-2}$, at a frequency $(f)$ of $304 \mathrm{kHz}$ and a load resistor of $1 \mathrm{k} \Omega$ (see Fig. 41(b)). Potential applications of LF-UEH for charging energy storage devices were also demonstrated, which exhibited a better charging power compared to other UEHs (see Fig. 41(c)). ${ }^{249-251}$ Finally, they conducted the evaluation of the LF-PUEH device in the ex vivo experiment of an implanted environment (see Fig. 41(d)). The output voltage showed relatively high retention (72-75\%) in an implanted environment (see Fig. 41(d)), indicating the favorable electrical properties. Therefore, KNN-based ceramics with NPB showed great potential to be integrated on implanted biomedical devices for electrical stimulation applications.

\section{Summary, challenges, and outlook}

\subsection{Summary}

As one of the most promising lead-free piezoelectric candidates, KNN-based ceramics have attracted more and more attention. Very recently, the construction of the NPB greatly improved the piezoelectric properties of KNN-based ceramics and accelerated the step towards practical applications. Herein, this work systematically reviewed the development and advance of the NPB in KNN-based ceramics, particularly focusing on the construction of the NPB, the effects of the NPB on the piezoelectric properties, strain properties, and temperature stability of KNN-based ceramics as well as their related physical mechanisms. Particularly, each part of the performance was systematically discussed with regard to the recent advances and physical mechanisms. Subsequently, the validity of the NPB was also convincingly proved by reviewing other results. Finally, the incipient devices prepared by using KNN-based ceramics with NPB were also introduced, which showed promising performance for practical applications. Therefore, this review can promote the understanding of 
KNN-based ceramics with NPB and the design of further KNNbased ceramics.

\subsection{Challenges}

Although the recent work proved that KNN-based ceramics with NPB are highly promising for partially replacing the lead-based ones, there are still challenges for the development of KNNbased ceramics and their practical applications.

6.2.1 Physical mechanisms. Although the NPB could be constructed empirically, the related physical mechanisms of the enhanced performance are still not well understood. As discussed in Section 3.1.3, the investigations on in situ observations for phase structure and domain configuration are key to understand the physical mechanism at the NPB. But however, the related study is rarely reported so far. Therefore, the future work can concentrate on this by using some advanced tools, such as in situ XRD, in situ Raman spectra and in situ TEM. ${ }^{192,252}$ Furthermore, a similar NPB exhibited distinct differences in piezoelectric properties (e.g., $d_{33}$ values varied from $400 \mathrm{pC} \mathrm{N}^{-1}$ to $650 \mathrm{pC} \mathrm{N}^{-1}$ ). Thus, the exact ability of the NPB to improve performance is also strongly dependent on the choice of additives. These additives affected the phase and microstructure of KNN-based ceramics' NPB with regard to the electronegativity, ionic radius, chemical valence, and so on. ${ }^{16}$ Therefore, a deeper analysis at the atom/nanoscale is highly desired to clearly understand the physical mechanisms. Once the physical mechanisms are disclosed, a more effective instructional relationship of "composition design-phase/micro structure-macro performance" can be obtained, which could further guide the development of KNN-based ceramics' NPB.

6.2.2 Stability. Up to now, small signal $d_{33}$ values of KNNbased ceramics with NPB could reach as high as $400-650 \mathrm{pC} \mathrm{N}^{-1}$ comparable to those of commercial soft PZT-based ceramics. But however, the intrinsic trait of the PPB also inevitably endows the NPB with temperature dependence in both phase structure and performance, which greatly hinders the practical applications of KNN-based ceramics. Although the strain properties exhibited improved temperature stability under a high electric field, the practical applications for actuators require a low electric field. Therefore, it is important to improve the temperature stability of strain properties under low electric fields without significantly sacrificing their performance. Moreover, the current understanding of the temperature stability of KNN-based ceramics is still indistinct. Thus, an in-depth analysis of their physical mechanism is urgently needed. In addition to temperature stability, fatigue stability and mechanical stability are also important factors for practical applications. To resolve this challenge, one may concentrate on the composition design, fabrication technology and other methods (e.g., composite ceramic, metallic oxide). The composition design could guarantee benign electrical properties, while the fabrication technology and other methods could improve the stability of KNN-based ceramics.

6.2.3 Reproducibility. The most reported NPB was constructed by doping three or more other elements into KNN ceramics. Such doping will reduce the reproducibility of KNN-based ceramics because of the many factors, such as the volatilization of the alkali metal, the high sensitivity of sintering temperature and the chemical inhomogeneity. As mentioned above, even the raw materials also greatly affect the structure and performance of KNN-based ceramics. More importantly, the reproducibility should be further improved from the viewpoint of industrial applications because of too many elements involved. Industrial applications require mass production. When carrying out mass production, many other unexpected challenges may come out, such as cost and chemical inhomogeneity. To resolve this challenge, the precise record of raw materials and fabrication technology is highly required. In addition, the refinement of fabrication technology is still needed.

\subsection{Outlook}

Though challenges exist, KNN-based ceramics with NPB are still the most promising candidates due to the high performance. Considering the current development of KNN-based ceramics with NPB, future research should focus on the following investigations.

(1) Applying ordered structures with functional units (OSFU) initially proposed by Chen $e t$ al. in KNN-based ceramics with NPB. ${ }^{253}$ The OSFU concept emphasizes the effects of the functional units of materials on the performance, while the previous research mainly focused on the relationship of structure and performance. ${ }^{253}$ Actually, this concept has been implemented in relaxor- $\mathrm{PbTiO}_{3}$ piezoelectric material systems. ${ }^{81,91,111,170}$ For example, Li et al. firstly systematically revealed that the OSFU consisting of long-range ordered ferroelectric domains and PNRs played an important role in enhancing the piezoelectric properties of relaxor- $-\mathrm{PbTiO}_{3}$ single crystals. ${ }^{81,111}$ Then, they further elaborately controlled the OSFU by chemical modification, and then achieved higher piezoelectric properties in relaxor- $\mathrm{PbTiO}_{3}$ ceramics $\left(d_{33}=1510 \mathrm{pC} \mathrm{N}{ }^{-1}\right)$ and single crystals $\left(d_{33}=3400-4100 \mathrm{pC} \mathrm{N}^{-1}\right)$, the highest $d_{33}$ values in piezoelectric ceramics and single crystals reported so far. ${ }^{91,170}$ Therefore, the investigations and applications of OSFU in KNNbased ceramics with NPB will further improve the performance, which is currently absent but one of the most promising future research directions.

(2) Further enriching the database of effective additives for constructing the NPB. As mentioned above, the NPB provides the fundamental basis for achieving high piezoelectric properties, while the elaborate chemical modification will further determine the obtained performance. Thus, it is necessary and meaningful to further enrich the database of effective additives for pursing better piezoelectric properties.

(3) Deeply studying the relationship of "chemical modificationstructure-performance-physical mechanism". As addressed in challenges, the physical mechanisms of enhanced piezoelectric properties are still needed to be further studied. The future investigations should not only focus on the physical mechanisms but also systematically unveil the foregoing relationship. Once the relationship is clear, KNN-based ceramics would take an important step forward toward the industrial applications.

(4) Further investigating the effects of preparation technology on the performance at the NPB. As discussed in Sections 3.1.2 and 4 , other optimized methods, such as the two-step sintering method and the two-step fabrication method, could further improve the piezoelectric properties of KNN-based ceramics. 
That is to say, a better performance is highly expected if one further optimizes the preparation technology of KNN-based ceramics with NPB. Unfortunately, there are only a few publications about this, which is one of the future research directions of KNN-based ceramics with NPB.

(5) Further preparing the incipient devices. The present high-performance of KNN-based ceramics with NPB allows us to tentatively prepare $\mathrm{KNN}$-based ceramics with the purpose of replacing partly lead-based ones in electronic devices. More importantly, the good co-sintering of KNN-based ceramics with base metals (e.g., $\mathrm{Cu}$ and $\mathrm{Ni}$ ) further proves the promising potential for industrial applications. ${ }^{254,255}$ Therefore, we believe that the NPB will promote the development of $\mathrm{KNN}$-based ceramics in both scientific research and industrial applications in the future.

\section{Conflicts of interest}

There are no conflicts to declare.

\section{Acknowledgements}

We gratefully acknowledge the support from the National Natural Science Foundation of China (NSFC No. 51722208 and 51972215) and the Key Technologies Research and Development Program of Sichuan Province (No. 2018JY0007). We thank Prof. Jürgen Rödel (Technische Universität Darmstadt) for measuring in situ temperature-dependent small signal $d_{33}$ values.

\section{References}

1 B. Jaffe, Piezoelectric ceramics, Elsevier, 2012.

2 T. R. Shrout and S. J. Zhang, J. Electroceram., 2007, 19, 113-126.

3 S.-E. Park and T. R. Shrout, J. Appl. Phys., 1997, 82, 1804-1811.

4 C. A. Randall, A. Kelnberger, G. Yang, R. Eitel and T. R. Shrout, J. Electroceram., 2005, 14, 177-191.

5 J. Rödel, W. Jo, K. T. Seifert, E. M. Anton, T. Granzow and D. Damjanovic, J. Am. Ceram. Soc., 2009, 92, 1153-1177.

6 J. F. Li, K. Wang, F. Y. Zhu, L. Q. Cheng and F. Z. Yao, J. Am. Ceram. Soc., 2013, 96, 3677-3696.

7 Y. Xu, Ferroelectric materials and their applications, Elsevier, 2013.

8 P. Panda and B. Sahoo, Ferroelectrics, 2015, 474, 128-143.

9 J. Rödel, K. G. Webber, R. Dittmer, W. Jo, M. Kimura and D. Damjanovic, J. Eur. Ceram. Soc., 2015, 35, 1659-1681.

10 J. Wu, D. Xiao and J. Zhu, Chem. Rev., 2015, 115, 2559-2595.

11 C.-H. Hong, H.-P. Kim, B.-Y. Choi, H.-S. Han, J. S. Son, C. W. Ahn and W. Jo, J. Materiomics, 2016, 2, 1-24.

12 M. Acosta, N. Novak, V. Rojas, S. Patel, R. Vaish, J. Koruza, G. Rossetti Jr and J. Rödel, Appl. Phys. Rev., 2017, 4, 041305.

13 J. Koruza, A. J. Bell, T. Frömling, K. G. Webber, K. Wang and J. Rödel, J. Materiomics, 2018, 4, 13-26.

$14 \mathrm{~J}$. Wu, Advances in Lead-free Piezoelectric Materials, Springer, 2018.

15 T. Zheng, J. Wu, D. Xiao and J. Zhu, Prog. Mater. Sci., 2018, 98, 552-624.
16 Y. Zhang and J.-F. Li, J. Mater. Chem. C, 2019, 7, 4284-4303. 17 E. Sun and W. Cao, Prog. Mater. Sci., 2014, 65, 124-210.

18 J. Gao, D. Xue, W. Liu, C. Zhou and X. Ren, Actuators, 2017, 6, 24.

19 W. Liu and X. Ren, Phys. Rev. Lett., 2009, 103, 257602.

20 X. Liu and X. Tan, Adv. Mater., 2016, 28, 574-578.

21 L. Egerton and D. M. Dillon, J. Am. Ceram. Soc., 1959, 42, 438-442.

22 Y. Saito, H. Takao, T. Tani, T. Nonoyama, K. Takatori, T. Homma, T. Nagaya and M. Nakamura, Nature, 2004, 432, 84-87.

23 K. Xu, J. Li, X. Lv, J. Wu, X. Zhang, D. Xiao and J. Zhu, Adv. Mater., 2016, 28, 8519-8523.

24 H. Tao, H. Wu, Y. Liu, Y. Zhang, J. Wu, F. Li, X. Lyu, C. Zhao, D. Xiao and J. Zhu, J. Am. Chem. Soc., 2019, 141, 13987-13994.

25 T. Zheng, H. Wu, Y. Yuan, X. Lv, Q. Li, T. Men, C. Zhao, D. Xiao, J. Wu and K. Wang, Energy Environ. Sci., 2017, 10, 528-537.

26 B. Wu, H. Wu, J. Wu, D. Xiao, J. Zhu and S. J. Pennycook, J. Am. Chem. Soc., 2016, 138, 15459-15464.

27 M.-H. Zhang, K. Wang, Y.-J. Du, G. Dai, W. Sun, G. Li, D. Hu, H. C. Thong, C. Zhao and X.-Q. Xi, J. Am. Chem. Soc., 2017, 139, 3889-3895.

28 X. Lv, J. Wu, S. Yang, D. Xiao and J. Zhu, ACS Appl. Mater. Interfaces, 2016, 8, 18943-18953.

29 Q. Liu, Y. Zhang, J. Gao, Z. Zhou, H. Wang, K. Wang, X. Zhang, L. Li and J.-F. Li, Energy Environ. Sci., 2018, 11, 3531-3539.

30 X. Lv and J. Wu, J. Mater. Chem. C, 2019, 7, 2037-2048.

31 X. Lv, J. Wu, C. Zhao, D. Xiao, J. Zhu, Z. Zhang, C. Zhang and X.-X. Zhang, J. Eur. Ceram. Soc., 2019, 39, 305-315.

32 X. Sun, J. Zhang, X. Lv, Y. Liu, F. Li and J. Wu, J. Mater. Chem. A, 2019, 7, 16803-16811.

33 M. Wu, T. Zheng, H. Zheng, J. Li, W. Wang, M. Zhu, F. Li, G. Yue, Y. Gu and J. Wu, J. Mater. Chem. A, 2018, 6, 16439-16449.

34 L. Jiang, R. Chen, J. Xing, G. Lu, R. Li, Y. Jiang, K. Kirk Shung, J. Zhu and Q. Zhou, J. Appl. Phys., 2019, 125, 214501.

35 L. Jiang, Y. Yang, R. Chen, G. Lu, R. Li, J. Xing, K. K. Shung, M. S. Humayun, J. Zhu and Y. Chen, Adv. Funct. Mater., 2019, 1902522.

36 Q. Ke, W. H. Liew, H. Tao, J. Wu and K. Yao, IEEE Trans. Ultrason. Ferroelectr. Freq. Control, 2019, 66, 1395-1401.

37 J. Wu, Z. Fan, D. Xiao, J. Zhu and J. Wang, Prog. Mater. Sci., 2016, 84, 335-402.

38 J. Hao, W. Li, J. Zhai and H. Chen, Mater. Sci. Eng., R, 2019, 135, 1-57.

39 M. H. Lee, D. J. Kim, J. S. Park, S. W. Kim, T. K. Song, M. H. Kim, W. J. Kim, D. Do and I. K. Jeong, Adv. Mater., 2015, 27, 6976-6982.

40 R. Zuo, J. Fu and D. Lv, J. Am. Ceram. Soc., 2009, 92, 283-285. 41 X. Cheng, J. Wu, X. Wang, B. Zhang, J. Zhu, D. Xiao, X. Wang and X. Lou, Appl. Phys. Lett., 2013, 103, 052906.

42 B. Zhang, J. Wu, X. Cheng, X. Wang, D. Xiao, J. Zhu, X. Wang and X. Lou, ACS Appl. Mater. Interfaces, 2013, 5, 7718-7725. 
43 X. Cheng, J. Wu, X. Wang, B. Zhang, X. Lou, X. Wang, D. Xiao and J. Zhu, ACS Appl. Mater. Interfaces, 2013, 5, 10409-10417.

44 X. Wang, J. Wu, D. Xiao, J. Zhu, X. Cheng, T. Zheng, B. Zhang, X. Lou and X. Wang, J. Am. Chem. Soc., 2014, 136, 2905-2910.

45 T. Zheng, J. Wu, X. Cheng, X. Wang, B. Zhang, D. Xiao, J. Zhu, X. Wang and X. Lou, J. Mater. Chem. C, 2014, 2, 8796-8803.

46 M.-H. Zhang, K. Wang, J.-S. Zhou, J.-J. Zhou, X. Chu, X. Lv, J. Wu and J.-F. Li, Acta Mater., 2017, 122, 344-351.

47 R. Zuo, J. Fu, D. Lv and Y. Liu, J. Am. Ceram. Soc., 2010, 93, 2783-2787.

48 Y. Lv, C. Wang, J. Zhang, L. Wu, M. Zhao and J. P. Xu, Mater. Res. Bull., 2009, 44, 284-287.

49 R. Zuo, C. Ye and X. Fang, Jpn. J. Appl. Phys., 2007, 46, 6733.

50 B. Zhang, J. Wu, X. Wang, X. Cheng, J. Zhu and D. Xiao, Curr. Appl. Phys., 2013, 13, 1647-1650.

51 R. Wang, H. Bando, T. Katsumata, Y. Inaguma, H. Taniguchi and M. Itoh, Phys. Status Solidi RRL, 2009, 3, 142-144.

52 R. Wang, H. Bando, M. Kidate, Y. Nishihara and M. Itoh, Jpn. J. Appl. Phys., 2011, 50, 09ND10.

53 W. Liang, W. Wu, D. Xiao and J. Zhu, J. Am. Ceram. Soc., 2011, 94, 4317-4322.

54 H. Tao, J. Wu, T. Zheng, X. Wang and X. Lou, J. Appl. Phys., 2015, 118, 044102.

55 Y. Yuan, J. Wu, H. Tao, X. Lv, X. Wang and X. Lou, J. Appl. Phys., 2015, 117, 084103.

56 H. Du, W. Zhou, D. Zhu, L. Fa, S. Qu, Y. Li and Z. Pei, J. Am. Ceram. Soc., 2008, 91, 2903-2909.

57 C. Zhang, T. Zheng and J. Wu, Ceram. Int., 2016, 42, 16049-16054.

58 K. Wang and J. F. Li, Adv. Funct. Mater., 2010, 20, 1924-1929.

59 X. Lv, J. Wu, D. Xiao, J. Zhu and X. Zhang, Acta Mater., 2017, 140, 79-86.

60 X. Lv, J. Wu, D. Xiao, J. Zhu and X. Zhang, J. Am. Ceram. Soc., 2018, 101, 1191-1200.

61 Y. Dai, X. Zhang and G. Zhou, Appl. Phys. Lett., 2007, 90, 262903.

62 Q. Liu, J.-F. Li, L. Zhao, Y. Zhang, J. Gao, W. Sun, K. Wang and L. Li, J. Mater. Chem. C, 2018, 6, 1116-1125.

63 X. Lv, J. Li, T.-L. Men, J. Wu, X.-X. Zhang, K. Wang, J.-F. Li, D. Xiao and J. Zhu, ACS Appl. Mater. Interfaces, 2018, 10, 30566-30573.

64 X. Lv, J. Wu, D. Xiao, H. Tao, Y. Yuan, J. Zhu, X. Wang and X. Lou, Dalton Trans., 2015, 44, 4440-4448.

65 X. Lv, Z. Li, J. Wu, J. Xi, M. Gong, D. Xiao and J. Zhu, Mater. Des., 2016, 109, 609-614.

66 X. Lv, J. Wu, D. Xiao, J. Zhu, J. Zhang and X. x. Zhang, Adv. Electron. Mater., 2018, 4, 1800205.

67 Y. Zhang, L. Li, B. Shen and J. Zhai, Dalton Trans., 2015, 44, 7797-7802.

68 C. Zhou, J. Zhang, W. Yao, X. Wang, D. Liu and X. Sun, J. Appl. Phys., 2018, 124, 164101.

69 D. Liu, X. Zhang, W. Su, X. Wang, W. Yao, C. Zhou and J. Zhang, J. Alloys Compd., 2019, 779, 800-804.

70 K. Wang, F. Z. Yao, W. Jo, D. Gobeljic, V. V. Shvartsman, D. C. Lupascu, J. F. Li and J. Rödel, Adv. Funct. Mater., 2013, 23, 4079-4086.
71 F. Rubio-Marcos, J. F. Fernandez, D. A. Ochoa, J. E. García, R. E. Rojas-Hernandez, M. Castro and L. Ramajo, J. Eur. Ceram. Soc., 2017, 37, 3501-3509.

72 J. Zhang, X. Sun, W. Su, W. Yao and C. Zhou, Scr. Mater., 2020, 176, 108-111.

73 W. Yao, J. Zhang, C. Zhou, D. Liu and W. Su, J. Eur. Ceram. Soc., 2019, DOI: 10.1016/j.jeurceramsoc.2019.11.014.

74 H. Liu, P. Veber, A. Zintler, L. Molina-Luna, D. Rytz, M. Maglione and J. Koruza, IEEE Trans. Ultrason. Ferroelectr. Freq. Control, 2018, 65, 1508-1516.

75 J. Xing, Z. Tan, L. Jiang, Y. Wu, Y. Yue, Q. Chen, J. Wu, W. Zhang, D. Xiao and J. Zhu, Appl. Phys. Lett., 2017, 110, 022905.

76 J. Xing, Z. Tan, X. Chen, L. Jiang, W. Wang, X. Deng, B. Wu, J. Wu, D. Xiao and J. Zhu, Inorg. Chem., 2018, 58, 428-438.

77 J. Xing, Z. Tan, L. Xie, L. Jiang, J. Yuan, Q. Chen, J. Wu, W. Zhang, D. Xiao and J. Zhu, J. Am. Ceram. Soc., 2018, 101, 1632-1645.

78 W. D. Callister, Fundamentals of materials science and engineering, Wiley, London, 2000.

79 M. Barsoum and M. Barsoum, Fundamentals of ceramics, CRC press, 2002.

80 M. Acosta, Strain Mechanisms in Lead-Free Ferroelectrics for Actuators, Springer, 2016.

81 F. Li, S. Zhang, T. Yang, Z. Xu, N. Zhang, G. Liu, J. Wang, J. Wang, Z. Cheng and Z. G. Ye, Nat. Commun., 2016, 7, 13807.

82 C. Zhao, H. Wu, F. Li, Y. Cai, Y. Zhang, D. Song, J. Wu, X. Lyu, J. Yin and D. Xiao, J. Am. Chem. Soc., 2018, 140, 15252-15260.

83 H. Liu, P. Veber, J. Rödel, D. Rytz, P. B. Fabritchnyi, M. I. Afanasov, E. A. Patterson, T. Frömling, M. Maglione and J. Koruza, Acta Mater., 2018, 148, 499-507.

84 C. Zhou, J. Zhang, W. Yao, D. Liu and W. Su, Scr. Mater., 2019, 162, 86-89.

85 W. Yao, J. Zhang, X. Wang, C. Zhou, X. Sun and J. Zhan, J. Eur. Ceram. Soc., 2019, 39, 287-294.

86 P. Li, J. Zhai, B. Shen, S. Zhang, X. Li, F. Zhu and X. Zhang, Adv. Mater., 2018, 30, 1705171.

87 J. Gao, D. Xue, Y. Wang, D. Wang, L. Zhang, H. Wu, S. Guo, H. Bao, C. Zhou and W. Liu, Appl. Phys. Lett., 2011, 99, 092901.

88 J. P. Morniroli and J. W. Steeds, Ultramicroscopy, 1992, 45, 219-239.

89 J. Li, L. Fei and S. Zhang, J. Am. Ceram. Soc., 2014, 97, 1-27.

90 H. Fu and R. E. Cohen, Nature, 2000, 403, 281.

91 F. Li, D. Lin, Z. Chen, Z. Cheng, J. Wang, C. Li, Z. Xu, Q. Huang, X. Liao and L. Q. Chen, Nat. Mater., 2018, 17, 349.

92 L. Fan, J. Chen, Y. Ren, Z. Pan, L. Zhang and X. Xing, Phys. Rev. Lett., 2016, 116, 027601.

93 D. You, W. Jung, S. Choi and Y. Cho, Appl. Phys. Lett., 2004, 84, 3346-3348.

94 J. Li, R. Rogan, E. Üstündag and K. Bhattacharya, Nat. Mater., 2005, 4, 776.

95 K. A. Schönau, L. A. Schmitt, M. Knapp, H. Fuess, R.-A. Eichel, H. Kungl and M. J. Hoffmann, Phys. Rev. B: Condens. Matter Mater. Phys., 2007, 75, 184117.

96 R. Theissmann, L. A. Schmitt, J. Kling, R. Schierholz, K. A. Schönau, H. Fuess, M. Knapp, H. Kungl and M. J. Hoffmann, J. Appl. Phys., 2007, 102, 024111. 
97 C. Zhao, B. Wu, K. Wang, J.-F. Li, D. Xiao, J. Zhu and J. Wu, J. Mater. Chem. A, 2018, 6, 23736-23745.

98 Y. Qin, J. Zhang, Y. Gao, Y. Tan and C. Wang, J. Appl. Phys., 2013, 113, 84.

99 R. López-Juárez, O. Novelo-Peralta, F. González-García and F. Rubio-Marcos, J. Eur. Ceram. Soc., 2011, 31, 1861-1864.

100 P. Marton, I. Rychetsky and J. Hlinka, Phys. Rev. B: Condens. Matter Mater. Phys., 2010, 81, 144125.

101 Z.-H. Zhao, Y. Dai and F. Huang, Sustainable Mater. Technol., 2019, e00092.

102 J. Fu, R. Zuo and Z. Xu, Appl. Phys. Lett., 2011, 99, 062901.

103 Q. Liu, Y. Zhang, L. Zhao, J. Gao, Z. Zhou, K. Wang, X. Zhang, L. Li and J.-F. Li, J. Mater. Chem. C, 2018, 6, 10618-10627.

104 Y. Qin, J. Zhang, W. Yao, C. Lu and S. Zhang, ACS Appl. Mater. Interfaces, 2016, 8, 7257-7265.

105 H. Wang, J. Zhu, N. Lu, A. Bokov, Z.-G. Ye and X. Zhang, Appl. Phys. Lett., 2006, 89, 042908.

106 H. Wang, J. Zhu, X. W. Zhang, Y. X. Tang and H. S. Luo, J. Am. Ceram. Soc., 2008, 91, 2382-2384.

107 Y. Sato, T. Hirayama and Y. Ikuhara, Phys. Rev. Lett., 2011, 107, 187601.

108 J. Yao, W. Ge, L. Luo, J. Li, D. Viehland and H. Luo, Appl. Phys. Lett., 2010, 96, 222905.

109 H. Yu, H. Zeng, R. Chu, G. Li, H. Luo, Z. Xu and Q. Yin, J. Phys. D: Appl. Phys., 2004, 37, 2914.

110 J. Gao, Y. Hao, S. Ren, T. Kimoto, M. Fang, H. Li, Y. Wang, L. Zhong, S. Li and X. Ren, J. Appl. Phys., 2015, 117, 084106.

111 F. Li, S. Zhang, Z. Xu and L. Q. Chen, Adv. Funct. Mater., 2017, 27, 1700310.

112 T. Zheng and J. Wu, J. Mater. Chem. A, 2015, 3, 6772-6780.

113 X. Lv, J. Wu, J. Zhu, D. Xiao and X. Zhangb, J. Eur. Ceram. Soc., 2017, 38, 85-94.

114 T. Zheng and J. Wu, ACS Appl. Mater. Interfaces, 2016, 8, 9242-9246.

115 H. Tao and J. Wu, J. Mater. Chem. C, 2017, 5, 1601-1606.

116 X. Wang, J. Wu, X. Cheng, B. Zhang, D. Xiao, J. Zhu, X. Wang and X. Lou, J. Phys. D: Appl. Phys., 2013, 46, 495305.

117 C. Liu, D. Xiao, T. Huang, J. Wu, F. Li and J. Zhu, Ceram. Int., 2014, 40, 7589-7593.

118 H. Tao, J. Wu, D. Xiao, J. Zhu, X. Wang and X. Lou, ACS Appl. Mater. Interfaces, 2014, 6, 20358-20364.

119 X. Wang, J. Wu, D. Xiao, X. Cheng, T. Zheng, X. Lou, B. Zhang and J. Zhu, ACS Appl. Mater. Interfaces, 2014, 6, 6177-6180.

120 X. Wang, J. Wu, D. Xiao, X. Cheng, T. Zheng, B. Zhang, X. Lou and J. Zhu, J. Mater. Chem. A, 2014, 2, 4122-4126.

121 J. Wu, X. Wang, X. Cheng, T. Zheng, B. Zhang, D. Xiao, J. Zhu and X. Lou, J. Appl. Phys., 2014, 115, 114104.

122 J. Wu, Y. Yang, X. Wang, D. Xiao and J. Zhu, J. Mater. Sci.: Mater. Electron., 2014, 25, 4650-4656.

123 T. Zheng, J. Wu, X. Cheng, X. Wang, B. Zhang, D. Xiao, J. Zhu and X. Lou, Dalton Trans., 2014, 43, 9419-9426.

124 T. Zheng, J. Wu, X. Cheng, X. Wang, B. Zhang, D. Xiao, J. Zhu, X. Lou and X. Wang, Dalton Trans., 2014, 43, 11759-11766.

125 D. Mazhao, D. Xiao, J. Wu and J. Zhu, J. Mater. Sci.: Mater. Electron., 2015, 26, 7309-7315.
126 Z. Tan, J. Zhu, Y. Zhang, J. Xing, Q. Chen, B. Wu, L. Sun, J. Wu, L. Jiang and D. Xiao, Ceram. Int., 2015, 41, 14610-14614.

127 H. Tao, J. Wu, T. Zheng, X. Wang and X. Lou, J. Appl. Phys., 2015, 118, 044102.

128 J. Wu, B. Zhang and W. Wu, J. Alloys Compd., 2015, 651, 302-307.

129 T. Zheng, J. Wu, D. Xiao, J. Zhu, X. Wang and X. Lou, ACS Appl. Mater. Interfaces, 2015, 7, 20332-20341.

130 T. Zheng, J. Wu, D. Xiao, J. Zhu, X. Wang and X. Lou, J. Mater. Chem. A, 2015, 3, 1868-1874.

131 T. Zheng, J. Wu, D. Xiao, J. Zhu, X. Wang, L. Xin and X. Lou, ACS Appl. Mater. Interfaces, 2015, 7, 5927-5937.

132 T. Zheng, Y. Zu and J. Wu, J. Alloys Compd., 2015, 647, 927-934.

133 J.-S. Zhou, K. Wang, F.-Z. Yao, T. Zheng, J. Wu, D. Xiao, J. Zhu and J.-F. Li, J. Mater. Chem. C, 2015, 3, 8780-8787.

134 L. Jiang, J. Xing, Z. Tan, J. Wu, Q. Chen, D. Xiao and J. Zhu, J. Mater. Sci., 2016, 51, 4963-4972.

135 Z. Tan, J. Xing, L. Jiang, L. Sun, J. Wu, W. Zhang, D. Xiao and J. Zhu, RSC Adv., 2016, 6, 6983-6989.

136 H. Tao and J. Wu, J. Eur. Ceram. Soc., 2016, 36, 1605-1612.

137 H. Tao, W. Wu and J. Wu, J. Alloys Compd., 2016, 689, 759-766.

138 R. Xiang and J. Wu, J. Alloys Compd., 2016, 684, 397-402.

139 J. Xing, Z. Tan, L. Jiang, Q. Chen, J. Wu, W. Zhang, D. Xiao and J. Zhu, J. Appl. Phys., 2016, 119, 034101.

140 L. Jiang, Y. Li, J. Xing, J. Wu, Q. Chen, H. Liu, D. Xiao and J. Zhu, Ceram. Int., 2017, 43, 2100-2106.

141 F. Li, Q. Gou, J. Xing, Z. Tan, L. Jiang, L. Xie, J. Wu, W. Zhang, D. Xiao and J. Zhu, J. Mater. Sci.: Mater. Electron., 2017, 28, 18090-18098.

142 F. Li, Z. Tan, J. Xing, L. Jiang, B. Wu, J. Wu, D. Xiao and J. Zhu, J. Mater. Sci.: Mater. Electron., 2017, 28, 8803-8809.

143 X. Lv, J. Wu, J. Zhu and D. Xiao, Phys. Chem. Chem. Phys., 2018, 20, 20149-20159.

144 X. Lv, J. Wu, J. Zhu, D. Xiao and X. Zhang, J. Eur. Ceram. Soc., 2018, 38, 85-94.

145 X. Lv, J. Wu, J. Zhu, D. Xiao and X. x. Zhang, J. Am. Ceram. Soc., 2018, 101, 4084-4094.

146 W. Liang, Z. Wang, D. Xiao, J. Wu, W. Wu, T. Huang and J. Zhu, Integr. Ferroelectr., 2012, 139, 63-74.

147 X. Cheng, J. Wu, X. Wang, B. Zhang, J. Zhu, D. Xiao, X. Wang, X. Lou and W. Liang, J. Appl. Phys., 2013, 114, 124107.

148 X. Cheng, Q. Gou, J. Wu, X. Wang, B. Zhang, D. Xiao, J. Zhu, X. Wang and X. Lou, Ceram. Int., 2014, 40, 5771-5779.

149 X. Cheng, J. Wu, X. Lou, X. Wang, X. Wang, D. Xiao and J. Zhu, ACS Appl. Mater. Interfaces, 2014, 6, 750-756.

150 X. Cheng, J. Wu, X. Wang, B. Zhang, J. Zhu, D. Xiao, X. Wang and X. Lou, Dalton Trans., 2014, 43, 3434-3442.

151 X. Cheng, J. Wu, T. Zheng, X. Wang, B. Zhang, D. Xiao, J. Zhu, X. Wang and X. Lou, J. Alloys Compd., 2014, 610, 86-91.

152 T. Huang, D. Xiao, C. Liu, F. Li, B. Wu, J. Wu and J. Zhu, Ceram. Int., 2014, 40, 2731-2735.

153 C. Liu, D. Xiao, T. Huang, J. Wu, F. Li, B. Wu and J. Zhu, Mater. Lett., 2014, 120, 275-278. 
154 H. Wang, J. Wu, X. Cheng, D. Xiao and J. Zhu, J. Alloys Compd., 2014, 585, 748-752.

155 X. Wang, J. Wu, X. Lv, H. Tao, X. Cheng, T. Zheng, B. Zhang, D. Xiao and J. Zhu, J. Mater. Sci.: Mater. Electron., 2014, 25, 3219-3225.

156 Z. Wang, D. Xiao, J. Wu, M. Xiao, F. Li and J. Zhu, J. Am. Ceram. Soc., 2014, 97, 688-690.

157 S. Feng, D. Xiao, J. Wu, M. Xiao and J. Zhu, J. Alloys Compd., 2015, 619, 560-563.

158 Q. Gou, D.-Q. Xiao, B. Wu, M. Xiao, S.-S. Feng, D.-D. M. Zhao, J.-G. Wu and J.-G. Zhu, RSC Adv., 2015, 5, 30660-30666.

159 H. Wang and J. Wu, J. Alloys Compd., 2015, 628, 329-334.

160 R. Wang, K. Wang, F. Yao, J. F. Li, F. H. Schader, K. G. Webber, W. Jo and J. Rödel, J. Am. Ceram. Soc., 2015, 98, 2177-2182.

161 B. Wu, J. Wu, D. Xiao and J. Zhu, Dalton Trans., 2015, 44, 21141-21152.

162 J. Xing, Z. Tan, J. Yuan, L. Jiang, Q. Chen, J. Wu, W. Zhang, D. Xiao and J. Zhu, RSC Adv., 2016, 6, 57210-57216.

163 Z. Tan, J. Xing, B. Wu, J. Wu, J. Zhu and D. Xiao, J. Mater. Sci.: Mater. Electron., 2017, 28, 12851-12857.

164 M.-H. Zhang, K. Wang, J.-S. Zhou, J.-J. Zhou, X. Chu, X. Lv, J. Wu and J.-F. Li, Acta Mater., 2017, 122, 344-351.

165 Q. Gou, J. Zhu, J. Wu, F. Li, L. Jiang and D. Xiao, J. Alloys Compd., 2018, 730, 311-317.

166 Y. Yao, C. Zhou, D. Lv, D. Wang, H. Wu, Y. Yang and X. Ren, EPL, 2012, 98, 27008.

167 G. Fan, W. Lu, X. Wang and F. Liang, Appl. Phys. Lett., 2007, 91, 202908.

168 D. Lin, Q. Zheng, C. Xu and K. Kwok, Appl. Phys. A: Mater. Sci. Process., 2008, 93, 549.

169 D. Lin and K. W. Kwok, J. Am. Ceram. Soc., 2010, 93, 806-813.

170 F. Li, M. J. Cabral, B. Xu, Z. Cheng, E. C. Dickey, J. M. LeBeau, J. Wang, J. Luo, S. Taylor and W. Hackenberger, Science, 2019, 364, 264-268.

171 D. Wang, K. Zhu, H. Ji and J. Qiu, Ferroelectrics, 2009, 392, 120-126.

172 J. Fang, X. Wang, Z. Tian, C. Zhong, L. Li and R. Zuo, J. Am. Ceram. Soc., 2010, 93, 3552-3555.

173 H. Du, F. Tang, F. Luo, W. Zhou, S. Qu and Z. Pei, Mater. Sci. Eng., B, 2007, 137, 175-179.

$174 \mathrm{~J}$. Wu, Y. Wang and H. Wang, RSC Adv., 2014, 4, 64835-64842.

175 F.-Z. Yao, K. Wang, W. Jo, J.-S. Lee and J.-F. Li, J. Appl. Phys., 2014, 116, 114102.

176 J. P. Praveen, T. Karthik, A. James, E. Chandrakala, S. Asthana and D. Das, J. Eur. Ceram. Soc., 2015, 35, 1785-1798.

177 D. Song, M. S. Woo, J. H. Ahn and T. H. Sung, J. Korean Phys. Soc., 2015, 66, 1549-1553.

178 C. Qiu, J. Liu, F. Li and Z. Xu, J. Appl. Phys., 2019, 125, 014102.

179 F. Rubio-Marcos, J. J. Romero, D. A. Ochoa, J. E. García, R. Perez and J. F. Fernandez, J. Am. Ceram. Soc., 2010, 93, 318-321.

180 P. Zheng, J. Zhang, Y. Tan and C. Wang, Acta Mater., 2012, 60, 5022-5030.
181 D. Ghosh, A. Sakata, J. Carter, P. A. Thomas, H. Han, J. C. Nino and J. L. Jones, Adv. Funct. Mater., 2014, 24, 885-896.

182 V. Hornebecq, C. Huber, M. Maglione, M. Antonietti and C. Elissalde, Adv. Funct. Mater., 2004, 14, 899-904.

183 W. Yang, P. Li, S. Wu, F. Li, B. Shen and J. Zhai, Adv. Electron. Mater., 2019, 1900570.

184 D. Damjanovic, J. Am. Ceram. Soc., 2005, 88, 2663-2676.

185 M. Davis, D. Damjanovic and N. Setter, J. Appl. Phys., 2006, 100, 084103.

186 F. Li, S. Zhang, Z. Xu, X. Wei, J. Luo and T. R. Shrout, J. Appl. Phys., 2010, 108, 034106.

187 F. Li, S. Zhang, Z. Xu, X. Wei, J. Luo and T. R. Shrout, Appl. Phys. Lett., 2010, 96, 192903.

188 F. Li, L. Jin, Z. Xu and S. Zhang, Appl. Phys. Rev., 2014, 1, 011103.

189 K. Uchino, S. Nomura, L. E. Cross, R. E. Newnham and S. J. Jang, J. Mater. Sci., 1981, 16, 569-578.

190 D. Vanderbilt and M. H. Cohen, Phys. Rev. B: Condens. Matter Mater. Phys., 2001, 63, 094108.

191 K. Brajesh, K. Tanwar, M. Abebe and R. Ranjan, Phys. Rev. B: Condens. Matter Mater. Phys., 2015, 92, 224112.

192 H. Guo, C. Zhou, X. Ren and X. Tan, Phys. Rev. B: Condens. Matter Mater. Phys., 2014, 89, 100104.

193 Y. Qin, J. Zhang, Y. Tan, W. Yao, C. Wang and S. Zhang, J. Eur. Ceram. Soc., 2014, 34, 4177-4184.

194 S. O. Leontsev and R. E. Eitel, Sci. Technol. Adv. Mater., 2010, 11, 044302.

195 W. Jo, R. Dittmer, M. Acosta, J. Zang, C. Groh, E. Sapper, K. Wang and J. Rödel, J. Electroceram., 2012, 29, 71-93.

196 S. Y. Choi, S. J. Jeong, D. S. Lee, M. S. Kim, J. S. Lee, J. H. Cho, B. I. Kim and Y. Ikuhara, Chem. Mater., 2012, 24, 3363-3369.

197 C. Zhao, B. Wu, H.-C. Thong and J. Wu, J. Eur. Ceram. Soc., 2018, 38, 5411-5419.

198 M. Hinterstein, M. Hoelzel, J. Rouquette, J. Haines, J. Glaum, H. Kungl and M. Hoffman, Acta Mater., 2015, 94, 319-327.

199 B. Narayan, J. S. Malhotra, R. Pandey, K. Yaddanapudi, P. Nukala, B. Dkhil, A. Senyshyn and R. Ranjan, Nat. Mater., 2018, 17, 427-431.

200 L. Jin, R. Huo, R. Guo, F. Li, D. W. Wang, Y. Tian, Q. Hu, X. Wei, Z. He and Y. Yan, ACS Appl. Mater. Interfaces, 2016, 8, 31109-31119.

201 C. Ang and Z. Yu, Adv. Mater., 2010, 18, 103-106.

202 J. Fu and R. Zuo, Acta Mater., 2013, 61, 3687-3694.

203 H. Liu, J. Chen, H. Huang, L. Fan, Y. Ren, Z. Pan, J. Deng, L.-Q. Chen and X. Xing, Phys. Rev. Lett., 2018, 120, 055501.

204 H. Liu, H. Huang, L. Fan, Y. Ren, H. Zhou, L.-Q. Chen, J. Chen and X. Xing, Phys. Rev. Mater., 2018, 2, 111403.

205 S. Trolier-McKinstry, S. Zhang, A. J. Bell and X. Tan, Annu. Rev. Mater. Res., 2018, 48, 191-217.

206 T. Leist, J. Chen, W. Jo, E. Aulbach, J. Suffner and J. Rödel, J. Am. Ceram. Soc., 2012, 95, 711-715.

207 F. Z. Yao, K. Wang, W. Jo, K. G. Webber, T. P. Comyn, J. X. Ding, B. Xu, L. Q. Cheng, M. P. Zheng and Y. D. Hou, Adv. Funct. Mater., 2016, 26, 1217-1224.

208 S.-T. Zhang, A. B. Kounga, E. Aulbach, W. Jo, T. Granzow, H. Ehrenberg and J. Rödel, J. Appl. Phys., 2008, 103, 034108. 
209 Z. Liu, B. Wu and J. Wu, Ceram. Int., 2018, 44, 16483-16488. 210 P. Li, X. Chen, F. Wang, B. Shen, J. Zhai, S. Zhang and Z. Zhou, ACS Appl. Mater. Interfaces, 2018, 10, 28772-28779.

211 P. Li, B. Liu, B. Shen, J. Zhai, Y. Zhang, F. Li and X. Liu, J. Eur. Ceram. Soc., 2018, 38, 75-83.

212 P. Li, Y. Huan, W. Yang, F. Zhu, X. Li, X. Zhang, B. Shen and J. Zhai, Acta Mater., 2019, 165, 486-495.

213 Q. Liu, Y. Zhang, J. Gao, Z. Zhou, D. Yang, K.-Y. Lee, A. Studer, M. Hinterstein, K. Wang and X. Zhang, Natl. Sci. Rev., 2019, DOI: 10.1093/nsr/nwz167.

214 J. Zhang, Z. Pan, F.-F. Guo, W.-C. Liu, H. Ning, Y. Chen, M.-H. Lu, B. Yang, J. Chen and S.-T. Zhang, Nat. Commun., 2015, 6, 6615 .

215 M. Acosta, L. A. Schmitt, L. Molina-Luna, M. C. Scherrer, M. Brilz, K. G. Webber, M. Deluca, H. J. Kleebe, J. Rödel and W. Donner, J. Am. Ceram. Soc., 2015, 98, 3405-3422.

216 D. Wang, F. Hussain, A. Khesro, A. Feteira, Y. Tian, Q. Zhao and I. M. Reaney, J. Am. Ceram. Soc., 2017, 100, 627-637.

217 F. Rubio-Marcos, R. López-Juárez, R. E. Rojas-Hernandez, A. del Campo, N. Razo-Perez and J. F. Fernandez, ACS Appl. Mater. Interfaces, 2015, 7, 23080-23088.

218 S.-A. Yang, B.-H. Kim, M.-K. Lee, S.-D. Bu and G.-J. Lee, Curr. Appl. Phys., 2016, 16, 593-598.

219 K. Zhang, Y. Guo, D. Pan, H. Duan, Y. Chen, H. Li and H. Liu, J. Alloys Compd., 2016, 664, 503-509.

220 F. Rubiomarcos, R. Lopezjuarez, R. E. Rojas Hernandez, C. A. Del, N. Razopérez and J. F. Fernandez, ACS Appl. Mater. Interfaces, 2015, 7, 23080-23088.

221 B.-H. Kim, S.-A. Yang, M.-K. Lee and G.-J. Lee, Ceram. Int., 2017, 43, 15880-15885.

222 D. Pan, Y. Guo, X. Fu, R. Guo, H. Duan, Y. Chen, H. Li and H. Liu, Solid State Commun., 2017, 259, 29-33.

223 D. Pan, Y. Guo, K. Zhang, H. Duan, Y. Chen, H. Li and H. Liu, J. Alloys Compd., 2017, 693, 950-954.

224 Z. Cen, W. Feng, P. Zhao, L. Chen, C. Zhu, Y. Yu, L. Li and X. Wang, J. Am. Ceram. Soc., 2019, 102, 2675-2683.

225 Z. Cen, Y. Zhen, W. Feng, L. Chen, P. Zhao, Y. Yu, M. Xiao, L. Li and X. Wang, J. Am. Ceram. Soc., 2019, 102, 3588-3597.

226 M.-K. Lee, S.-D. Bu and G.-J. Lee, Energies, 2019, 12, 886.

227 M.-K. Lee, S.-A. Yang, J.-J. Park and G.-J. Lee, Sci. Rep., 2019, 9, 4195.

228 Y. Yang, H. Wang, Y. Li, Q. Zheng, J. Liao, W. Jie and D. Lin, Dalton Trans., 2019, 48, 10676-10682.

229 H.-C. Thong, C. Zhao, Z.-X. Zhu, X. Chen, J.-F. Li and K. Wang, Acta Mater., 2019, 166, 551-559.

230 Y. Wang, K. Yao, X. Qin, M. S. Mirshekarloo, X. Liu and F. E. H. Tay, Adv. Electron. Mater., 2017, 3, 1700033.

231 Y. Wang, H. Wu, X. Qin, K. Yao, S. J. Pennycook and F. E. H. Tay, Adv. Electron. Mater., 2019, 5, 1800691.

232 A. Piorra, A. Petraru, H. Kohlstedt, M. Wuttig and E. Quandt, J. Appl. Phys., 2011, 109, 104101.
233 G. Kang, K. Yao and J. Wang, J. Am. Ceram. Soc., 2012, 95, 986-991.

234 J. Huang, Y. Zhang, H. Jiang, P. Fan, J. Xiao and H. Zhang, Ceram. Int., 2015, 41, 10506-10511.

235 K. Yao and F. E. H. Tay, IEEE Trans. Ultrason. Ferroelectr. Freq. Control, 2003, 50, 113-116.

236 J. Ou-Yang, B. Zhu, Y. Zhang, S. Chen, X. Yang and W. Wei, Appl. Phys. A: Mater. Sci. Process., 2015, 118, 1177-1181.

237 P. Sun, Q. Zhou, B. Zhu, D. Wu, C. Hu, J. M. Cannata, J. Tian, P. Han, G. Wang and K. K. Shung, IEEE Trans. Ultrason. Ferroelectr. Freq. Control, 2009, 56, 2760-2763.

238 Q. Zhou, X. Xu, E. J. Gottlieb, L. Sun, J. M. Cannata, H. Ameri, M. S. Humayun, P. Han and K. K. Shung, IEEE Trans. Ultrason. Ferroelectr. Freq. Control, 2007, 54, 668-675.

239 K. A. Snook, J.-Z. Zhao, C. H. Alves, J. M. Cannata, W.-H. Chen, R. J. Meyer, T. A. Ritter and K. K. Shung, IEEE Trans. Ultrason. Ferroelectr. Freq. Control, 2002, 49, 169-176.

240 D. Wu, R. Chen, Q. Zhou, K. Shung, D. Lin and H. Chan, Ultrasonics, 2009, 49, 395-398.

241 S. Lee, K. Lam, X. Zhang and H. Chan, Ultrasonics, 2011, 51, 811-814.

242 X. Yan, K. H. Lam, X. Li, R. Chen, W. Ren, X. Ren, Q. Zhou and K. K. Shung, IEEE Trans. Ultrason. Ferroelectr. Freq. Control, 2013, 60, 1272-1276.

243 M. Bah, F. Giovannelli, F. Schoenstein, C. Brosseau, J.-R. Deschamps, F. Dorvaux, L. Haumesser, E. Le Clezio and I. Monot-Laffez, Ultrasonics, 2015, 63, 23-30.

244 G. Feuillard, V. Loyau, L. T. H. Hue, T. Wurlitzer, E. Ringgaard, W. Wolny, B. Malic, M. Kosec, A. Barzegar and D. Damjanovic, IEEE Symp. Ultrason., 2003, 2, 1995-1998.

245 N. M. Hagh, B. Jadidian, E. Ashbahian and A. Safari, IEEE Trans. Ultrason. Ferroelectr. Freq. Control, 2008, 55, 214-224.

246 D. Lin and K. W. Kwok, Int. J. Appl. Ceram. Technol., 2011, 8, 684-690.

247 Z. Y. Shen, J. F. Li, R. Chen, Q. Zhou and K. K. Shung, J. Am. Ceram. Soc., 2011, 94, 1346-1349.

248 K.-B. Kim, D. K. Hsu, B. Ahn, Y.-G. Kim and D. J. Barnard, Ultrasonics, 2010, 50, 790-797.

249 L. Jiang, Y. Yang, R. Chen, G. Lu, R. Li, D. Li, M. S. Humayun, K. K. Shung, J. Zhu and Y. Chen, Nano Energy, 2019, 56, 216-224.

250 A. G. Fowler, S. Moheimani and S. Behrens, J. Microelectromech. Syst., 2014, 23, 1454-1462.

251 Y. Zhu, S. R. Moheimani and M. R. Yuce, IEEE Sens. J., 2010, 11, 155-161.

252 D. Schütz, M. Deluca, W. Krauss, A. Feteira, T. Jackson and K. Reichmann, Adv. Funct. Mater., 2012, 22, 2285-2294.

253 K. Chen and L. Li, Adv. Mater., 2019, 1901115.

254 L. Gao, H. Guo, S. Zhang and C. Randall, Actuators, 2016, 5, 8. 255 L. Gao, S. W. Ko, H. Guo, E. Hennig and C. A. Randall, J. Am. Ceram. Soc., 2016, 99, 2017-2023. 